WALDEN

UNIVERSITY

A higher degree. A higher purpose.

Walden University ScholarWorks

\title{
Contributors to the Development of Intercultural Competence in Nursing Students
}

Esther Zazzi

Walden University

Follow this and additional works at: https://scholarworks.waldenu.edu/dissertations

Part of the Higher Education Administration Commons, Higher Education and Teaching Commons, and the Nursing Commons

This Dissertation is brought to you for free and open access by the Walden Dissertations and Doctoral Studies Collection at ScholarWorks. It has been accepted for inclusion in Walden Dissertations and Doctoral Studies by an authorized administrator of ScholarWorks. For more information, please contact ScholarWorks@waldenu.edu. 


\title{
Walden University
}

\author{
College of Health Sciences
}

This is to certify that the doctoral dissertation by

\author{
Esther Zazzi
}

has been found to be complete and satisfactory in all respects, and that any and all revisions required by the review committee have been made.

Review Committee

Dr. Janice Long, Committee Chairperson, Nursing Faculty

Dr. Geri Schmotzer, Committee Member, Nursing Faculty

Dr. Mattie Burton, University Reviewer, Nursing Faculty

Chief Academic Officer

Eric Riedel, Ph.D.

Walden University

2019 


\begin{abstract}
by

Esther Zazzi

MScN, Cardiff University, 2011

BScN, Kalaidos Fachhochschule Gesundheit, 2008

\author{
Dissertation Submitted in Partial Fulfillment \\ of the Requirements for the Degree of \\ Doctor of Philosophy \\ Nursing
}

Contributors to the Development of Intercultural Competence in Nursing Students

Walden University

February 2019 


\begin{abstract}
Nurses deal more effectively with cultural diversity when they have an ethnorelative orientation toward cultural difference and commonality on the Intercultural Development Continuum, which was the theoretical framework of this study. Scholarly literature shows limited knowledge on what fosters nurses' intercultural development. Thus, this quantitative, retrospective study was the first investigation in health care in Switzerland conducted on nursing students' orientation on the Intercultural Development Inventory (IDI) and the relationship to student demographic variables. The sample for this secondary data analysis consisted of the IDI results from nursing students enrolled between 2010 and 2016 at the largest nursing college in Switzerland $(N=3,410)$ where the systematic integration of the development of intercultural competence into the curriculum began in 2010. Descriptive statistical analysis indicated that the students' developmental orientation (DO) toward cultural difference and commonality represented a mindset of minimization $(M=86.3, S D=13.7)$, whereas the orientation gap between the DO and the students' perceived orientation (PO) was high $(M=32.5)$, indicating that students believe they have higher intercultural competence than they have. Chi-squaretests revealed significant relationships between gender, age, and time living in another country and the students' PO and DO. The paired samples $t$ test demonstrated a higher end of program DO $(p=.01)$ compared to beginning DO scores, suggesting the program provided challenges that met the students' level of readiness for cultural development. The findings may affect positive social change by providing knowledge on contributors to development of intercultural competence that educators can use.
\end{abstract}


Contributors to the Development of Intercultural Competence in Nursing Students by

Esther Zazzi

\author{
MScN, Cardiff University, 2011 \\ BScN, Kalaidos Fachhochschule Gesundheit, 2008
}
Dissertation Submitted in Partial Fulfillment
of the Requirements for the Degree of
Doctor of Philosophy
Nursing

Walden University

February 2019 


\section{Acknowledgments}

I would like to thank my chair, Dr. Janice M. Long, for her professional guidance, very competent advice, qualified support and kind encouragement she has provided within the last months of writing this dissertation.

I would like to thank as well my committee member, Dr. Geri L. Schmotzer, who gave competent advice regarding the content of this dissertation.

All the members of staff at Walden University who taught me throughout the courses and residencies or helped me with getting the literature also deserve thanks as well as all my fellow students from whom I could learn within very inspiring discussions.

In particular, I appreciated highly that Dr. Leslie C. Hussey very supportively guided me through this doctoral journey at the residencies, and Dr. Leilani Endicott inspired me to conduct a secondary data analysis.

A special thank goes to the management of the Nursing College who allowed me to use the data for a secondary data analysis.

I would like to thank also Dr. Mitchell Hammer, President of IDI, LLC, who approved this study and supported it by providing a dissertation research discount on the price of the Qualifying Seminar to use the Intercultural Development Inventory.

Last but not least, I would like to express my gratitude to my husband and son for their continued encouragement and great support. Particularly, they reliably and skillfully helped me with any kind of technical challenge. 
Table of Contents

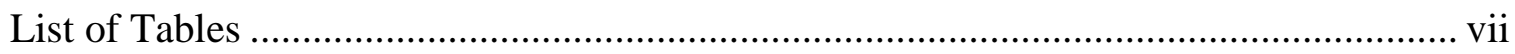

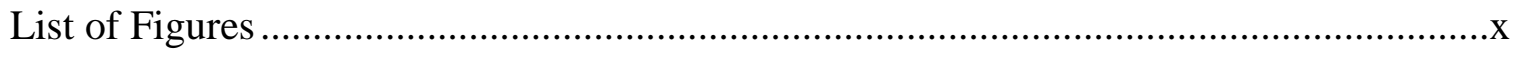

Chapter 1: Introduction to the Study ....................................................................

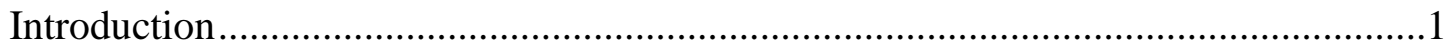

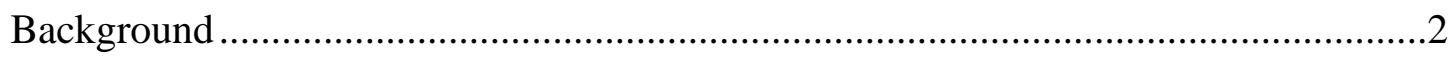

Influencing Factors of the Increasing Diversity in the Swiss Health Care

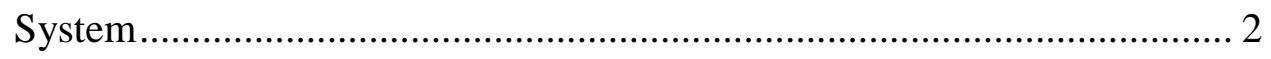

Marginalization and Discrimination Because of Diversity in Health Care ............ 6

Summary of Literature on Intercultural Competence ...................................... 8

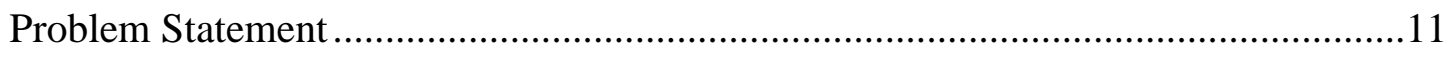

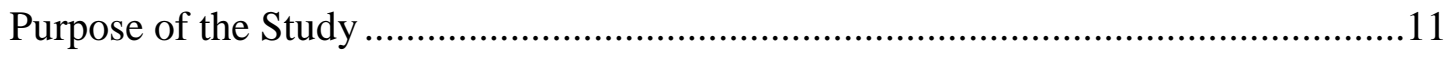

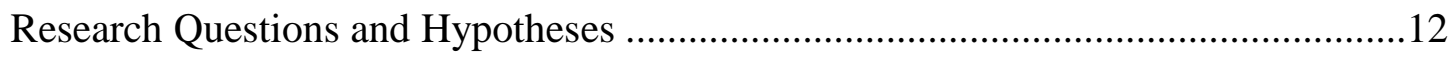

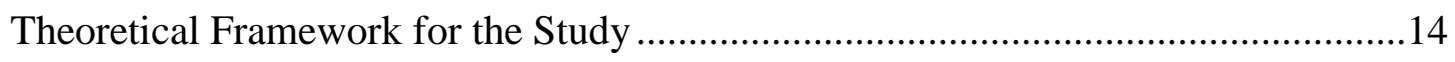

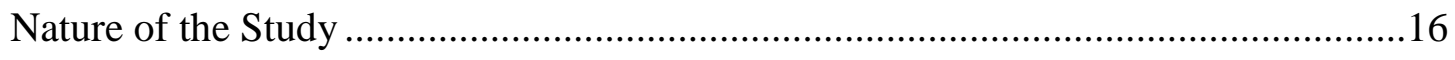

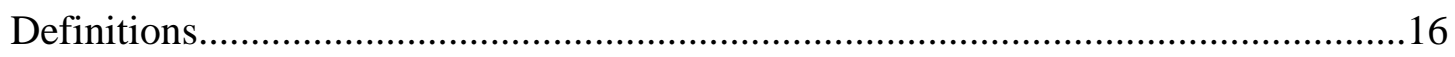

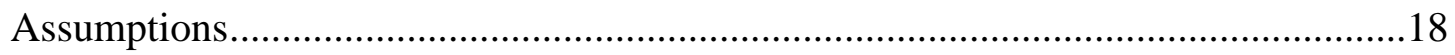

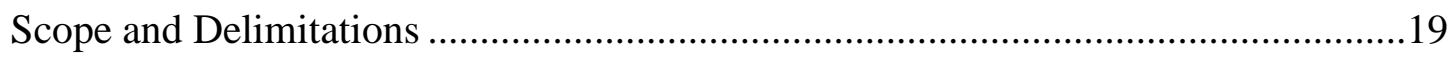

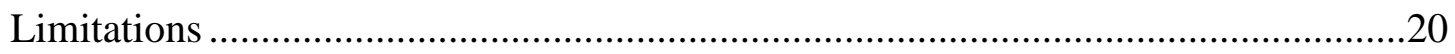

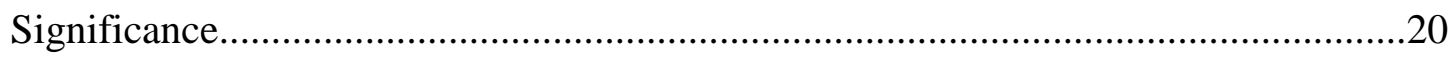

Contribution to Positive Social Change ...................................................... 22

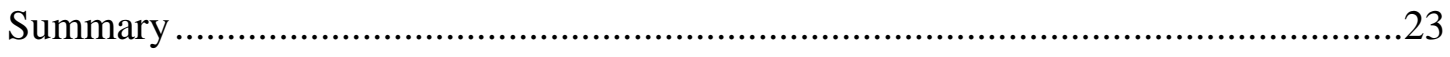




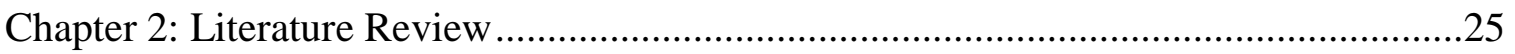

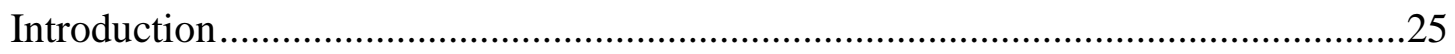

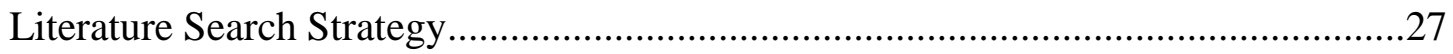

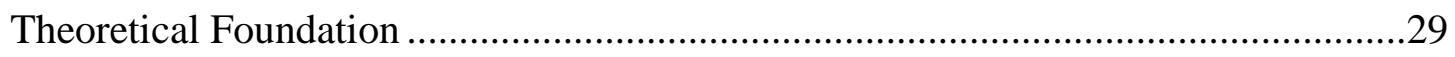

Origin on the Intercultural Development Continuum (IDC) ............................... 30

Rationale for the Choice of the IDC and the Theoretical Propositions ................ 32

Measurement of the Orientation Toward Cultural Difference and

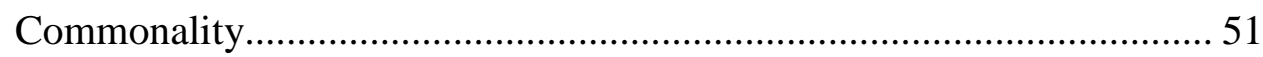

The Relation Between the Theory and the Present Study..................................... 52

Literature Review Related to the Key Variables .......................................................52

Dependent Variable Orientation Toward Cultural Difference and

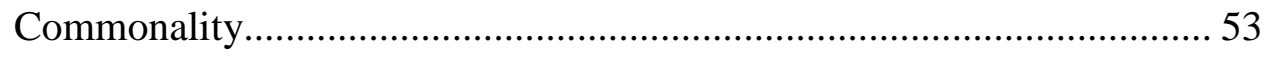

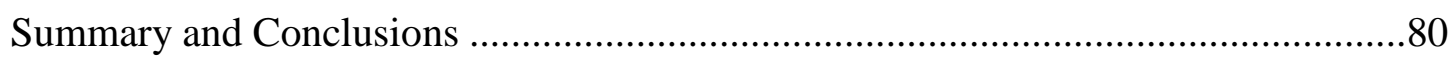

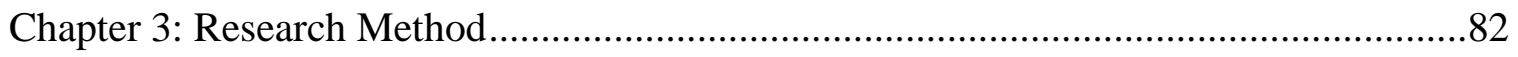

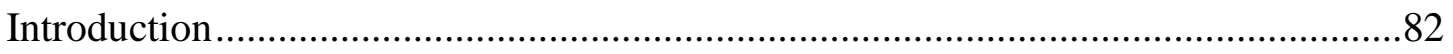

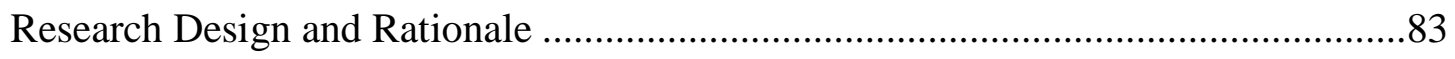

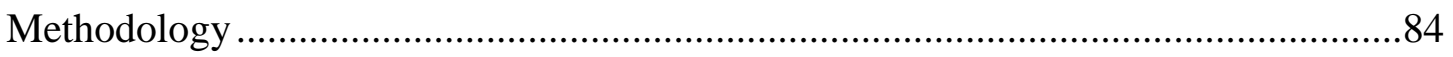

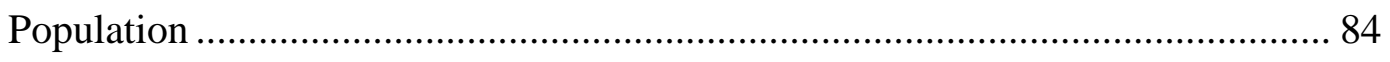

Sample

Procedure for Gaining Access to the Data Set.................................................... 85

Instrumentation and Operationalization of Constructs .......................................... 87

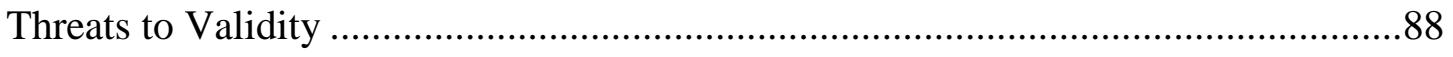


Ethical Procedures

Summary

Chapter 4: Results

Introduction

Data Collection

Baseline Descriptive and Demographic Characteristics of the Sample

Results to Research Question 1

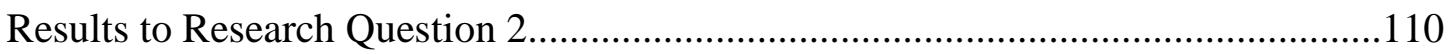

Relationship Between the Students' Age and Their PO ................................ 112

Relationship Between the Students' Age and Their Developmental

Orientation

Relationship Between the Students' Gender and Their Perceived

Orientation

Relationship Between the Students' Gender and Their Developmental

Orientation

Relationship Between the Students' Primary Country of Citizenship and

Their Perceived Orientation.

Relationship Between the Students' Primary Country of Citizenship and

Their Developmental Orientation

Relationship Between the Students' Ethnic Minority Group Affiliation and

Their Perceived Orientation. 
Relationship Between the Students' Ethnic Minority Group Affiliation and

Their Developmental Orientation

Relationship Between the Students' Migration Background and Their

Perceived Orientation.

Relationship Between the Students' Migration Background and Their

Developmental Orientation

Relationship Between the World Region Students Primarily Lived Up to

Age 18 and Their Perceived Orientation

Relationship Between the World Region Students Primarily Lived Up to

Age 18 and Their Developmental Orientation

Relationship Between the Total Amount of Time Students Lived in

Another Country and Their Perceived Orientation

Relationship Between the Total Amount of Time Students Lived in

Another Country and Their Developmental Orientation

Relationship Between the Amount of Lessons About Own Culture or Any

Foreign Cultures the Students Had and Their Perceived Orientation..... 130

Relationship Between the Amount of Lessons About Own Culture or Any

Foreign Cultures the Students Had and Their Developmental

Orientation

Relationship Between the Students' Previous Education as a Health Care Assistant and Their Perceived Orientation 133 
Relationship Between the Students' Previous Education as a Health Care

Assistant and Their Developmental Orientation

Summary the Relationships Between Perceived and Developmental

Orientation and Demographic Variables

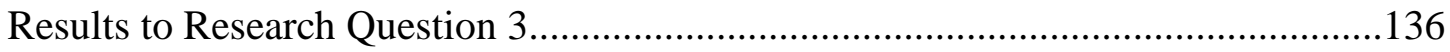

Difference Regarding Perceived Orientation................................................ 137

Difference Regarding Developmental Orientation ....................................... 138

Difference Regarding the Orientation Gap .................................................. 139

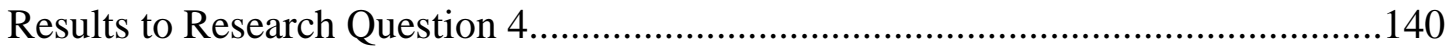

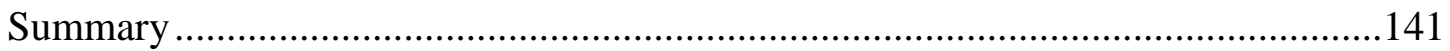

Chapter 5: Discussion, Conclusions, and Recommendations ....................................145

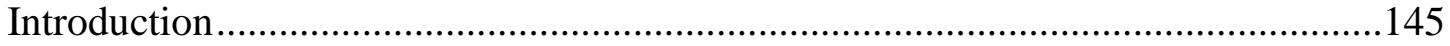

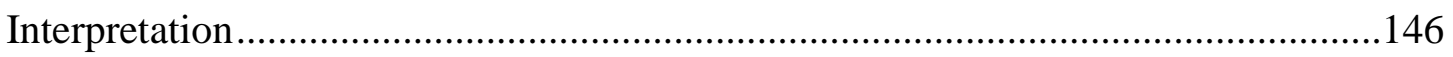

Orientation Toward Cultural Difference and Commonality of the Nursing

Students

Relationships Between the Nursing Students' Orientation and Their

Demographic Variables

151

Foster the Development of the Orientation Toward Cultural Difference and Commonality of the Nursing Students ...................................... 158

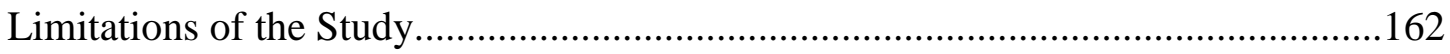

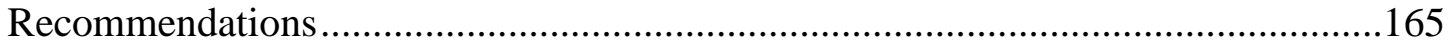

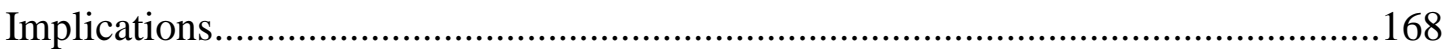




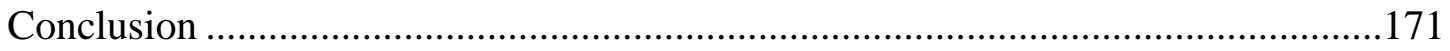

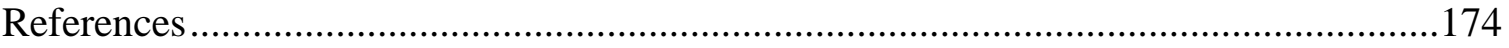

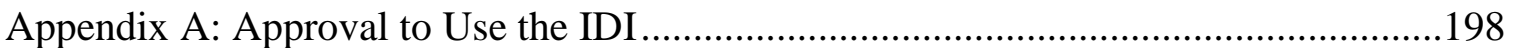

Appendix B: Number of IDI in Each Cohort Including Time-Point ...........................199 


\section{List of Tables}

Table 1. Keywords, Phrases, and Inclusion Criteria/Exclusion Criteria.......................... 28

Table 2. Seven Subthemes of Diversity by Leonard (2006) ........................................ 50

Table 3. Frequencies for Demographic Variables ...................................................... 97

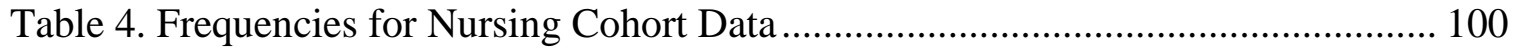

Table 5. Perceived Orientation at the Start-Point and End-Point of the Education ........ 103

Table 6. Developmental Orientation at the Start-Point and End-point of the Education 104

Table 7. Perceived Orientation of the Students at the Start-Point of the Education ....... 105

Table 8. Developmental Orientation of the Students at the Start-Point of the Education

Table 9. Perceived Orientation of the Students at the End-Point of the Education ........ 107

Table 10. Developmental Orientation of the Students at the End-Point of the Education

Table 11. Orientation Gap Between Perceived Orientation and Developmental Orientation at Start-and End-Point of the Education 110

Table 12. Observed and Expected Frequency of the Perceived Orientation for Age ..... 113

Table 13. Observed and Expected Frequency of Developmental Orientation for Students'

Age 114

Table 14. Observed and Expected Frequency of the Perceived Orientation for Students'

Gender.

Table 15. Observed and Expected Frequency of the Developmental Orientation for Students' Gender. 116 
Table 16. Observed and Expected Frequency of Perceived Orientation for Students'

Primary Country of Citizenship 117

Table 17. Observed and Expected Frequency of Developmental Orientation for Students' Primary Country of Citizenship ....................................................................... 118

Table 18. Observed and Expected Frequency of Perceived Orientation for Students'

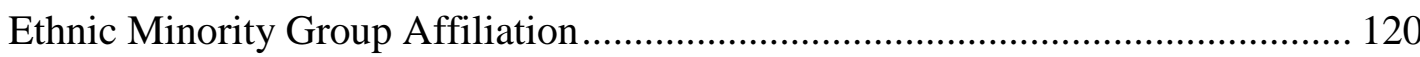

Table 19. Observed and Expected Frequency of the Developmental Orientation for the Students' Ethnic Minority Group Affiliation. 121

Table 20. Observed and Expected Frequency of Perceived Orientation for Students' Migration Background 122

Table 21. Observed and Expected Frequency of Developmental Orientation for Students' Migration Background 123

Table 22. Observed and Expected Frequency of Perceived Orientation for World Region Students Primarily Lived up to Age 18 124

Table 23. Observed and Expected Frequency of Developmental Orientation for World Regions Students Primarily Lived up to Age 18 125

Table 24. Observed and Expected Frequency of Perceived Orientation for Time Students Lived in Another Country 127

Table 25. Observed and Expected Frequency of Developmental Orientation for Time Students Lived in Another Country 128

Table 26. Observed and Expected Frequency of Perceived Orientation for Amount of Lessons on Culture. 130 
Table 27. Observed and Expected Frequency of Developmental Orientation for Amount of Lessons on Culture 131

Table 28. Observed and Expected Frequency of Perceived Orientation for Students' Previous Education as a Health Care Assistant

Table 29. Observed and Expected Frequency of Developmental Orientation for Students' Previous Education as a Health Care Assistant 134

Table 30. Paired Samples T Test of Perceived Orientation at Start Compared to End of Education

Table 31. Paired Samples T Test of Developmental Orientation at Start Compared to End of Education 138

Table 32. Paired Samples T Test of Orientation Gap at Start Compared to End of Education 139

Table 33. Number of Students Who Started or Completed Nursing Education 162 


\section{List of Figures}

Figure 1. Percentages of the permanent foreign population in Switzerland between 1900

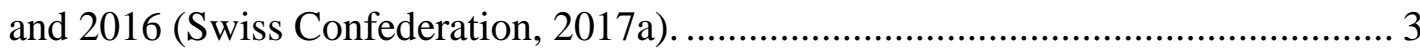

Figure 2. Main languages of the permanent resident population, 1970-2016 (Swiss

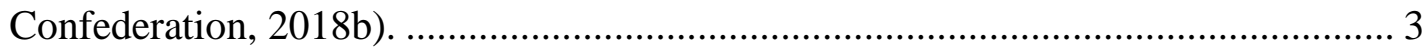

Figure 3. The religion of the Swiss population in the years 2014 - 2016 (Swiss

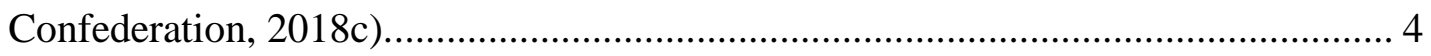

Figure 4. The evolution of the religious landscape (Swiss Confederation, 2018d).......... 5

Figure 5. The Intercultural Development Continuum (Hammer, 2012) ....................... 29

Figure 6. Distribution of the score of perceived orientation at the start-point and the endpoint of the education. 103

Figure 7. Distribution of the score of developmental orientation at the start-point and the end-point of the education.

Figure 8. Distribution of the score of perceived orientation at the start-point of the education 106

Figure 9. Distribution of the students' score of developmental orientation at the startpoint of the education. 107

Figure 10. Distribution of the students' score of perceived orientation at the end-point of the education. 108 
Chapter 1: Introduction to the Study

\section{Introduction}

Diversity within the Swiss health care system is growing as the population demographics change. According to the International Council of Nursing (2013), "nurses should be culturally and linguistically competent to understand and respond effectively to the cultural and linguistic needs of clients, families, and communities in a health care encounter" (p. 1). Therefore, it is important to understand nursing students' development of intercultural competence. Researchers have implemented various interventions to foster intercultural competence; however, methodological rigor and uniformity on core components of education interventions, including how they are described and evaluated, has been lacking. Therefore, there was a need to know more about contributors to intercultural competence, particularly within nursing.

Applying a secondary data analysis, this quantitative research study was an investigation into the effect of a specialized program integrated into the curriculum at a Nursing College in Switzerland on the nursing student-cohort's orientation toward cultural difference and commonality. Furthermore, the relationship between the individual nursing students' orientation toward cultural difference and commonality and the demographic factors of the nursing students (age, gender, country of citizenship, ethnic minority group affiliation, migration background, world region having primarily lived up to age 18 , total amount of time having lived in another country, having had lessons about own culture or any foreign cultures, and previous education as a health care assistant) was examined. This study may affect positive social change by providing more 
knowledge on the contributors to intercultural competence, which nursing educators may use to increase intercultural competence of nursing students.

The study will be introduced in this chapter with background information about the current knowledge on intercultural competence. The problem regarding the need for nurses to deal with the growing diversity in Switzerland will be stated before presenting the purpose of this study. The research questions and hypotheses, as well as the theoretical framework, will also be outlined. This chapter will also include the nature of the study, definitions, and the assumptions, scope and delimitations, and limitations. Finally, the significance of this study will be explained before a summary.

\section{Background}

\section{Influencing Factors of the Increasing Diversity in the Swiss Health Care System}

Increase of foreign population. Since 1960, the population of Switzerland has increased from just over 5 million inhabitants to over 8 million - a boom mostly driven by immigrants from Europe (Nguyen \& Mariani, 2014). Most foreigners residing permanently in Switzerland are European and mainly come from a European Union or a European Free Trade Association member state, with Italian, German, Portuguese and French nationals comprising most foreigners (Swiss Confederation, 2018a). Today, Switzerland has one of the highest percentages of foreign residents in the world - more than one in four people are of foreign origin (Statista, 2018). The percentages of the permanent foreign population in Switzerland between 1900 and 2016 are presented in Figure 1 (Swiss Confederation, 2017a). 


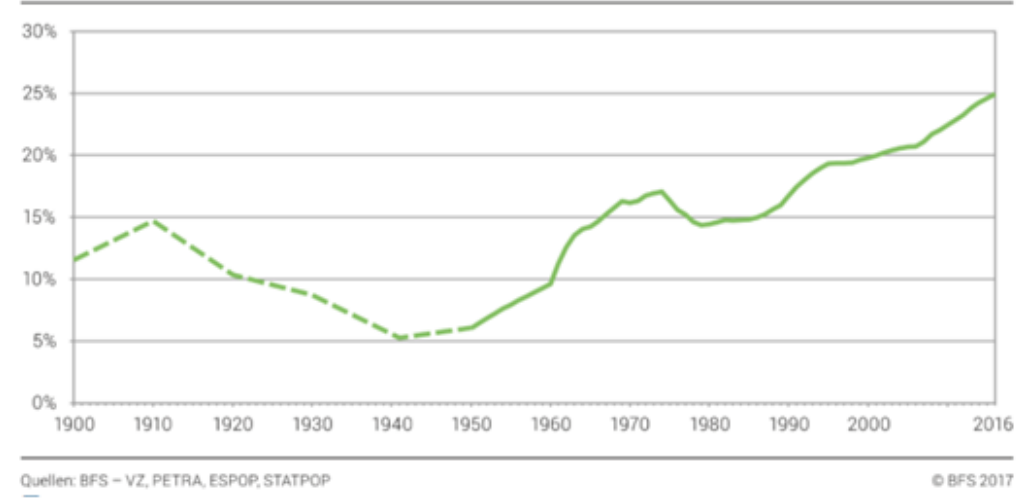

Figure 1. Percentages of the permanent foreign population in Switzerland between 1900 and 2016 (Swiss Confederation, 2017a).

Increase of linguistic diversity. Apart from foreign inhabitants with diverse nationalities, Swiss inhabitants need to speak one of the four official languages, traditionally spoken by Swiss in different regions of the country: German, French, Italian, and Rumantsch (All about Switzerland, 2016). Due to immigration, many individuals do not speak one of the four official languages of Switzerland as their first language (Swiss Confederation, 2018b). The percentages of the main languages of the permanent resident population, 1970-2016 are presented in Figure 2 (Swiss Confederation, 2018b).

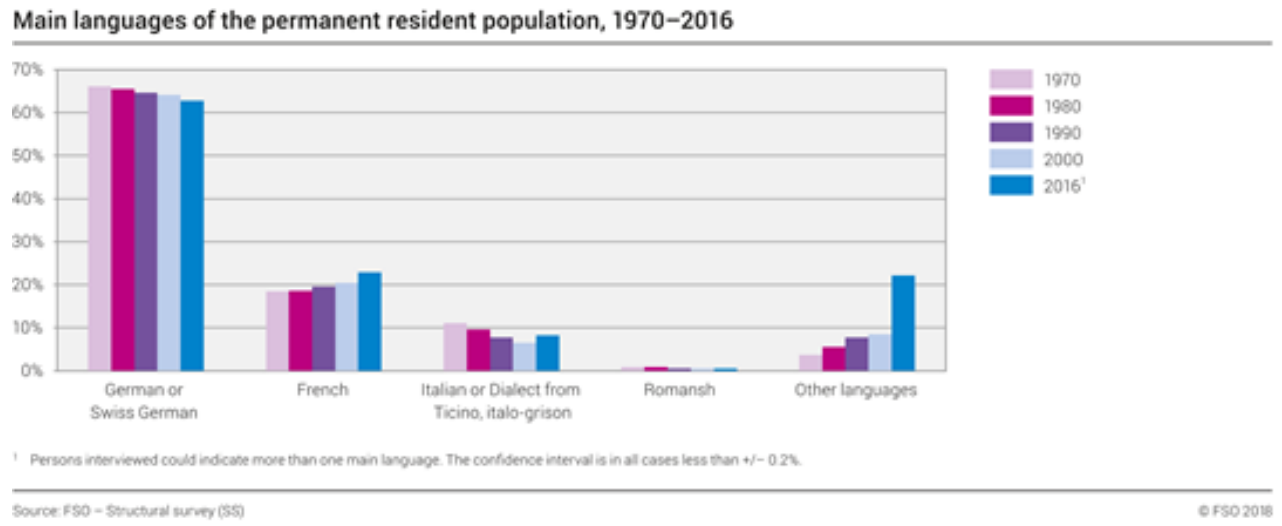

Figure 2. Main languages of the permanent resident population, 1970-2016 (Swiss Confederation, 2018b). 
Increase of religious diversity. With the freedom of religion as a basic constitutional right in Switzerland, there is an increasing diversity regarding religions in Switzerland (Swiss Confederation, 2018c). The religion of the Swiss population in the years 2014-2016 are presented in Figure 3 (Swiss Confederation, 2018c).

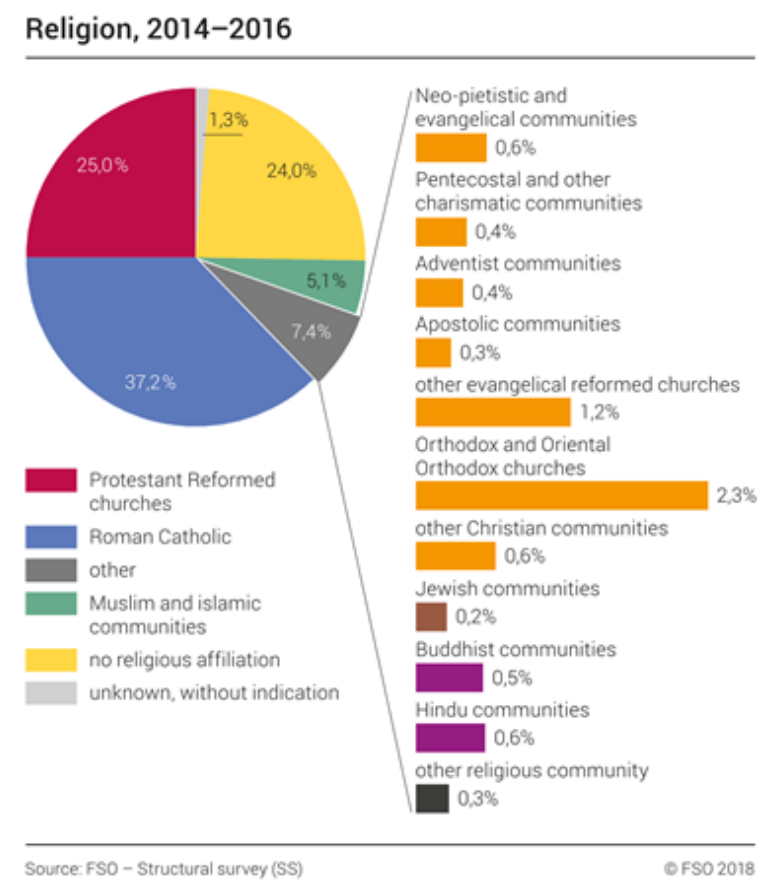

Figure 3. The religion of the Swiss population in the years 2014-2016 (Swiss Confederation, 2018c).

The population is not restricted to a religious denomination, and Muslim and Islamic communities as well as Christian communities (other than Protestant Reformed and Roman Catholic) have been increasing over the last decades (Swiss Confederation, 2018d). This evolution of the religious landscape in Switzerland is presented in Figure 4 (Swiss Confederation, 2018d). 


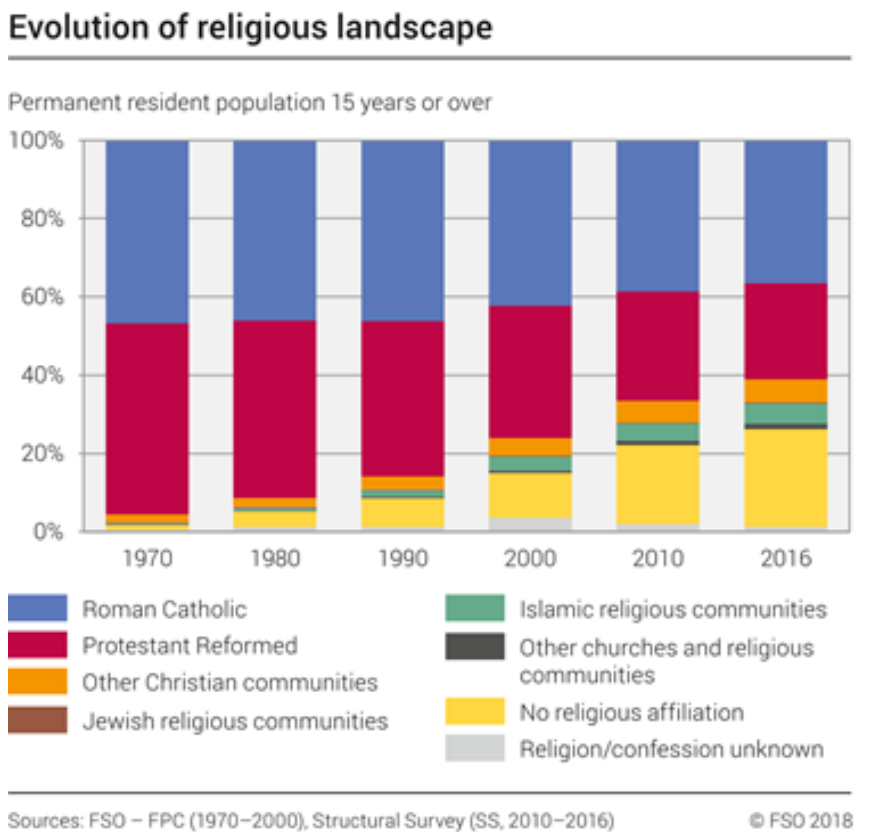

Figure 4. The evolution of the religious landscape (Swiss Confederation, 2018d).

Increase of elderly chronically-ill patients. There are also changes of demographics in Switzerland, as the population aged 65 and over increased from 191,593 persons in 1900 to 983,352 in 1990, and it was at 1.52 million in 2016 (Swiss Confederation, 2017b). According to the Swiss Confederation (2017b), this population group will grow in the coming years, as life expectancy at birth (i.e., the average number of years a person can expect to live) has risen from 76.2 in 1970 to 85.3 for women and from 70.1 to 81.5 for men. As the number of elderly people increases, so does the incidence of chronic diseases and the likelihood of illnesses such as cardiovascular diseases, rheumatism, cancer, or a combination of these conditions (Obsan, 2008). Therefore, the health care system is confronted with challenges to best cope with the increasing numbers of chronically-ill persons (Obsan, 2015; Wurz, 2014). 


\section{Diversification of grades in nursing professions and interprofessional}

collaborations. Although the changing demands on health care providers has increased, the education regulation of health professions in Switzerland has also changed with transitions in the last few years, especially in the field of nursing (OdASanté, 2017; Swiss Education, 2018). These changes have led to a diversification of grades within the nursing profession as well as new professional profiles (Mahrer, 2011). For example, there is now a 3-year apprenticeship at the secondary level for the profession of health care assistant (OdaSanté, 2018). This apprenticeship allows young people, 16 or older, to enter the health care system following their standard school curriculum and to graduate after 3 years of vocational education and training. Although not solely educated in the field of nursing, many of these young professionals are integrated into nursing teams, which challenges collaboration, something that is inherent in nursing (Bachmann, 2013; German Medical Science, 2014; University of Bern, 2014). The diversification of grades in the nursing profession and the requirement of collarboration in health care is accompanied by the diversity in health care providers. In summary, diversity is inherent to Swiss society regarding the increase of the foreign population, linguistic, and religious diversity, meaning there is diversity in the Swiss health care system not only in patients but also in health care providers (Nguyen \& Mariani, 2014; Swiss Confederation, 2017a, 2017b, 2018a, 2018b, 2018c; Wurz, 2014).

\section{Marginalization and Discrimination Because of Diversity in Health Care}

Diversity may lead to marginalization and discrimination, which may be accentuated in individuals who perceive they are different from the majority, not only 
because of ethnicity and language but also factors like age, gender, or disability. According to the Swiss Red Cross (2018), unreflective generalizations and prejudices by health professionals about individuals or whole groups of people prevent the view of their actual problems, which affects treatment and care. The Institute of Medicine (2002) reported that patients may be put at risk and even die because of health care providers' lack cultural competence that may lead to delay in treatment or noncompliance with health care regimes. Although health care professionals may not see themselves as overtly racist or neglectful, they could be missing pertinent health care findings due to cultural blindness (Seright, 2007). According to the Federal Office for Public Health (Swiss Confederation, 2018e), health professionals need to be trained to care for migrants living in Switzerland with their specific health-related needs due to their origin or their living situation to ensure quality of care and patient safety.

In terms of nursing education and diversity, research has shown the needs and challenges of students. Schuessler, Wilder, and Byrd (2012) concluded that self-reflection is necessary to be aware of cultural issues and address health care disparities. Similarly, McClimens, Brewster, and Lewis (2014) reported that nursing students struggle with meeting cultural needs of patients such as language, food, and gender.

In addition to education, health care professionals are also influenced by their own backgrounds and upbringing. Their cultural competence depends on their experiences that influence their perception of diversity and their related behavior such as opinions of their family, friends, and teachers. For example, Swedish and Slovenian health care professionals have exhibited a difference in teamwork, relationships between 
professions, and responses to patient problems (Pahor \& Rasmussen, 2009). Additionally, Cowan and Norman (2006) reported that migrant nurses from other European Union countries employed in London hospitals were perplexed by the diversity of cultures in the United Kingdom. Health care providers are also influenced by their environment such as the current situation regarding refugees from Syria and elsewhere in Europe ("Migration Crisis," 2015).

According to Bleich, MacWilliams \& Schmidt (2015), diversity has garnered increasing attention in a society that is demographically pluralistic. Research has shown that more than half of the Swiss population are xenophobic, and two-thirds want foreigners to be better integrated (Cattacin, Gerber, Sardi, \& Wegener, 2006; "Racism is also a Reality,” 2016). For example, in her opening speech at the Summer Institute for Intercultural Communication Dr. Janet Bennett showed posters and flyers of a campaign for an immigration initiative against criminal foreigners of Switzerland's largest political party showing a white sheep kicking a black sheep over the border (Schweizerische Volkspartei SVP, 2010; Swiss Confederation, 2018; The Intercultural Communication Institute, 2016a).

\section{Summary of Literature on Intercultural Competence}

The concept of intercultural competence has been explored in the literature for the last five decades (Deardorff, 2015). Several strategies to enhance intercultural competence in health care providers have been shown in smaller empirical research studies in the last 15 years. Although the findings of the empirical studies reporting the contributors to the proband's development of intercultural competence by using the 
Intercultural Development Inventory (IDI) are similar, the studies vary regarding intervention, duration, design, and scope.

Most researchers have developed their own training program. For example, Halm and Wilgus (2013) developed a training program in a traditional classroom setting focusing on Latino culture, including Spanish language/music, to highlight critical reflections, cross-cultural practice, and communication standards for culturally competent care. In a different approach, Harder (2018) applied a video and simulations with manikins to train culturally competent care. There are studies on cultural immersion but also various forms such as in-service session on the end-of-life care (Halm et al., 2012) or a 4-week-international placement (Peying et al., 2012). With such heterogeneity of interventions, the results may only be compared with consideration.

Researchers have also consistently emphasized the importance of reflection. However, reflection has not been clearly and comprehensively described, so interventions such as journal-writing or analysis of experiences within peer-discussion are heterogeneous as well. The same needs to be stated for the duration of the interventions and the time being devoted to the participants' development of the intercultural competence. Some researchers have allowed a short period of some weeks (e.g., Harder, 2018 or Peying et al., 2012), but other researchers have allowed 3 to 5 years (e.g., Huckabee \& Matkin, 2012 or Boggis, 2012). There are also considerations of researchers on how sustainable a certain development is. Researchers have debated whether a current intervention may increase participants' cultural awareness only short term (Halm \& 
Wilgus, 2013). Therefore, it remains unclear what is needed for an individual to develop further along the Intercultural Development Continuum (IDC) having a lasting effect.

Researchers have investigated with various health care professionals. The only identified studies using the IDI in nursing have been conducted by Halm and Wilgus (2013), Hamre (2012), Harder (2018), Kruse et al. (2014), Larson (2011), and Munoz et al. (2009). Some of them investigated among nursing students, nursing staff, nursing faculty or a mix of them. Bourjolly et al. (2015) and Halm et al. (2012) investigated within multidisciplinary teams including nurses. Furthermore, all studies in the literature review were conducted in the United States, except the study by Peying et al. (2012) that was conducted in Australia and the study by Harder (2018) that was conducted in Canada. No study using the IDI conducted in other parts of the word such as Europe has ever been published, including in fields outside of health care. Therefore, empirical research about the orientation toward cultural difference and commonality in nursing is limited, particularly in Europe including Switzerland.

Although several research studies have been conducted, the knowledge about what contributes to intercultural competence with health care providers is limited. For instance, Horvat, Horey, Romios, and Kis-Rigo (2014) concluded that "further research is required to establish greater methodological rigor and uniformity on core components of education interventions, including how they are described and evaluated" (p. 2). To foster the intercultural competence among nursing students, nursing educators need more knowledge to better understand the contributors to the development of intercultural competence of nursing students. Therefore, this study was conducted to address a need to 
know more about the contributors to the development of intercultural competence, especially in nursing in Switzerland.

\section{Problem Statement}

Although several studies have been conducted to identify what contributes to the development of the intercultural competence of nurses or other health care providers, knowledge is still limited. This limitation is related to the heterogeneity of the studies regarding content, scope, duration, design, and interventions. However, studies using the IDI have identified the participants within the developmental stage of minimization, the transition stage between the ethnocentric and the ethnorelative orientation toward difference and commonality. With such an orientation, health care providers minimize cultural differences, meaning they are limited in their ability to deal appropriately with diversity in health care. This study was conducted to address this and other concerns with the cultural competence of health care providers.

\section{Purpose of the Study}

The purpose of this quantitative and descriptive cross-sectional study was to identify the orientation toward cultural difference and commonality on the IDC of nursing students in Switzerland and to provide more knowledge about the contributors to the development of their intercultural competence. Accordingly, the effect of a specialized program integrated into the curriculum at a nursing college in Switzerland on the nursing student-cohorts' orientation toward cultural difference and commonality and its relationship to the cohorts' demographic variables was examined. Furthermore, the relationship between the nursing students' orientation toward cultural difference and 
commonality and the demographic variables of the individual nursing students (age, gender, country of citizenship, ethnic minority group affiliation, migration background, world region having primarily lived up to age 18 , total amount of time having lived in another country, having had lessons about own culture or any foreign cultures, and previous education as a health care assistant) was examined. This investigation was essential, as educators' knowledge of how individuals, teams, and organizations can increase intercultural competence can help achieve educational and organizational goals (Hammer, 2015b).

\section{Research Questions and Hypotheses}

The following questions and hypotheses led the secondary data analysis:

RQ 1: What are the nursing students' orientation toward cultural difference and commonality on the Intercultural Development Continuum?

RQ 2: What is the relationship among the nursing students' demographic variables and the nursing students' orientation toward cultural difference and commonality?

$H_{0}$ : There is no relationship among the nursing students' demographic variables and their stage on the Intercultural Development Continuum.

$H_{1}$ : There is a relationship among the nursing students' demographic variables and their stage on the Intercultural Development Continuum.

RQ 3: To what extent does the nursing student-cohorts' orientation toward cultural difference and commonality as a group differ from the beginning to the end of their education? 
RQ 4: What is the relationship between the difference regarding the nursing student-cohorts' orientation toward cultural difference and commonality at the start-point and end-pont of education and the demographic variables of the cohort (years of education, year of curriculum, workload, focus of specialization, and location of school)?

$H_{0}$ : There are no differences between the orientation toward cultural difference and commonality at the start of the nursing education and the orientation toward cultural difference and commonality at the end-point of the nursing education for the nursing student-cohorts according to the various demographics regarding duration of the education (2, 2.5, 3, or 4 years), year of curriculum (2010 or 2015), workload (full-time or short-time), focus of specialization (physically-ill patients, psychiatry, pediatrics/obstetrics/gynecology), location of school lessons (head location in a larger city or branch location in a smaller city in a mountainous area).

$H_{1}$ : There is a difference between the orientation toward cultural difference and commonality at the start of the nursing education and the orientation toward cultural difference and commonality at the end-point of the nursing education for the nursing student-cohorts according to the various demographics regarding duration of the education $(2,2.5,3$, or 4 years), year of curriculum (2010 or 2015), workload (full-time or short-time), focus of specialization (physically ill patients, psychiatry, pediatrics/obstetrics/gynecology), location of school lessons (head location in a larger city or branch location in a smaller city in a mountainous area).

The dependent variable was the individual nursing students' and the nursing student-cohorts' orientation toward cultural difference and commonality and was 
measured using the IDI (2018a, 2018b). The independent variables were the demographic variables of age, gender, country of citizenship, ethnic minority group affiliation, migration background, world region having primarily lived up to age 18 , total amount of time having lived in another country, having had lessons about own culture or any foreign cultures, and previous education as a health care assistant and the nursing studentcohorts' demographics such as duration of the education (2, 2.5, 3, or 4 years), curriculum (2010 or 2015), workload (full-time or short-time), focus of specialization (physically ill patients, psychiatry, pediatrics/obstetrics/ gynecology), and location of school lessons (head location in a larger city or branch location in a smaller city in a mountainous area). The nursing students at the nursing college completed the IDI (2018a, 2018b) between 2010 and 2016 at the start and the end of their 2, 2.5, 3, or 4-year education. The data for the dependent variables were provided by the nursing students in response to the 50 items in the IDI. The data for the independent variables were provided by the nursing students in response to additional questions within the IDI.

\section{Theoretical Framework for the Study}

The theoretical framework for this study was the IDC, which consists of the orientations toward cultural difference and commonality describing sets of knowledge, attitudes, and skills. The orientations are arrayed along a continuum from the more monocultural mindsets of denial and polarization through the transitional orientation of minimization to the intercultural or global mindsets of acceptance and adaptation (Hammer, 2012). The IDC is adapted from the Developmental Model of Intercultural Sensitivity (DMIS) originally proposed by Bennett (1986, 1993, 1998, 2001, 2004, 2009, 
2011, 2013), who explained how people experience and engage cultural differences in a continuum of experience extending from ethnocentrism to ethnorelativism.

Ethnocentrism is an individual's experience of personal culture as central to reality, and ethnorelativism is an individual's experience of personal and other cultures as relative to context. The developmental movement applies to anything defined as a cultural difference; it is permanent in one direction, but there may be retreats from some stages (Bennett, 2011).

Additionally, Hammer, Bennett, and Wisemann (2003) used the term intercultural sensitivity to refer to the ability to discriminate and experience the relevant cultural difference and the term intercultural competence to mean the ability to think and act in interculturally appropriate ways. Greater intercultural sensitivity is associated with greater potential for exercising intercultural competence, which involves adapting behavior to cultural differences (IDI, 2018b, p. 3). Intercultural competence reflects the degree to which cultural difference and commonality in values, expectations, beliefs, and practices are bridged, an inclusive environment is achieved, and specific differences that exist in an individual's organization are addressed from a "mutual adaptation" perspective (IDI, 2018b). Shifting cultural perspective and bridging behavior across cultural differences is best achieved by maintaining an adaptation perspective (Hammer, 2012). A more detailed explanations of the theoretical propositions of the IDC by Hammer (2012) based on the DMIS by Bennett (2011) is provided in Chapter 2. 


\section{Nature of the Study}

This study involved a quantitative and descriptive cross-sectional study design. A secondary data analysis was conducted by using data of nursing students at a nursing college in Switzerland who completed the IDI (2018a, 2018b) between 2010 and 2016 at the start and the end of their 2, 2.5, 3, or 4-year education. This study was an investigation into the effect of a specialized program integrated into the curriculum at a nursing college in Switzerland on the nursing student-cohorts' orientation toward cultural difference and commonality and its relationship to the cohorts' demographic variables. Furthermore, the relationship between the individual nursing students' orientation toward cultural difference and commonality and the demographic variables of the nursing students was examined. Statistical analysis methods were applied to identify correlations among the variables as this study was an examination of the contributors to the development of the intercultural competence of nursing students at a certain time. The results of the IDI the nursing students' orientations toward cultural difference and commonality including the stage on the IDC at the start and the end of their 2, 2.5, 3, or 4-year education was identified.

\section{Definitions}

Cultural difference and commonality: This term refers to the concepts of culture and diversity. This study was based on Bennett's (2001) definition of culture referring to the subjective culture: "The pattern of beliefs, behaviors, and values maintained by groups of interacting people." (p. 3). This definition emphasizes the worldview of different cultural groups, which is a prerequisite to deal more effectively with diversity. 
Diversity: Based on Hammer (2012) and Leonard (2006), the underlying definition of diversity of this study was broad and included but was not limited to the characteristics (a) race, ethnicity, nationality; (b) circumstances (including educational background, work, geographic home, economic situation, sexual orientation, family; (c) religion; (d) languages; and (e) health status or disabilities.

Educator: All professionals within the tertiary level professional education who contribute to the development or implementation of the nursing education were meant (Swiss Education, 2018). This included professionals who teach but also professionals who decide on the development of the education including the design of the curriculum.

Health care provider: Every professional who directly works with patients within health care (Obsan, 2016). With the term patient, every recipient of health care in all service areas (e.g., hospital, ambulatory, home care, nursing home) were meant (Swiss Confederation, 2018i).

Intercultural competence: The definition of intercultural competence for this study was: "The capability to accurately understand and adapt behavior to cultural difference and commonality" (IDI, 2018b, p. 3). Additionally, it is "the degree to which cultural difference and commonality in values, expectations, beliefs, and practices are effectively bridged, an inclusive environment is achieved, and the specific difference that exists in one's organization are addressed from a mutual adaptation perspective" (IDI, 2018b, p. 3). This competence is most fully achieved by maintaining an adaptation perspective (Hammer, 2012). 


\section{Assumptions}

My assumptions about culture, diversity and cultural difference were essential to consider for this study, as they were shaped by my experience working at the nursing college where fostering intercultural competence has been implemented in the curriculum since 2010. Although the faculty of the nursing college have never defined the term diversity, they defined the term culture in 2010 in relation to the definition of subjective culture by Bennett (1998):

Culture is a dynamic process. Culture includes learned and shared patterns of beliefs, behaviors, and values of groups of interacting people. The group itself define, communicate, maintain, adapt and further develop these patterns of beliefs, behaviors, and values. Cultural values and norms provide an identity for people and offer guidance in various areas of life, e.g., partnership, family, work, leisure, politics, business, health, disease, diet, and age.

It was also essential to consider my theoretical perspective and point of view that influences how I see the world and relationships to parts of the world (Guba \& Lincoln, 1994). My research questions demonstrated a postpositivistic theoretical lens perspective. For a postpositivistic paradigm, the aim of inquiry is an explanation, enabling the prediction and control of phenomena (Guba \& Lincoln, 1994). The criterion for progress in this paradigm is the capability of scientists to predict and control improving over time (Hesse, as cited in Guba \& Lincoln, 1994). The criterion for progress in the postpositivistic paradigm was consistent with my ontological and epistemological assumptions. My ontological assumption of worldview was a realist approach, meaning 
that "structures creating the world cannot be directly observed" (Wainwright, 1997, p. 1264). However, realism is grounded in the ontological assumption that "there is an objective world that is independent on our beliefs, perceptions, knowledge, theories, and explanations” (Hirani, Richter, \& Salami, 2018, p. 138). For my point of view, hidden mechanisms enables us to explain what we experience in the real world, which supported the epistemological assumption that there are multiple ways of knowing. Thus, realistic ontological and epistemological assumptions help to explain and understand social phenomena and state about causal relationships between social phenomena. Therefore, the aim of my research was to show different explanations for reality (Wainwright, 1997, p. 1264).

\section{Scope and Delimitations}

This study was focused on the orientation toward cultural difference and commonality with nursing students at a nursing college in Switzerland. Accordingly, the scope of this study included a sample of the entire population of nursing students at the college in Switzerland. As the nursing college is the largest college of higher education in nursing in Switzerland, with about 400 students to become registered nurses every year, the findings may be generalized to other nursing students at a college of higher education in nursing in Switzerland. However, other colleges have not implemented the development of the intercultural competence as extensively into their curriculum. Additionally, applying the post facto design investigating into data already collected the sample cannot be considered random (Simon \& Goes, 2013); therefore, a generalization of the findings needs careful consideration. 
Furthermore, the scope of this study the study was based on the IDC (Hammer, 2012), and the data of nursing students assessed by using the IDI as the measurement instrument (IDI, 2018a, 2018b). The data set consists of a considerable amount of data, which would not have been possible to collect within the scope of this dissertation Therefore, the perspective on the topic was shaped according to the propositions this theoretical framework is based on. As the development of the intercultural competence is complex, other propositions of other theories such as Campinha-Bacote's (2018) process of cultural competence in the delivery of health care services may have been neglected.

\section{Limitations}

The sample for the study consisted of the data collected from the nursing students at a nursing college, which represented a convenience sample. With this approach, the data were included because the students were enrolled at the nursing college between 2010 and 2016. Furthermore, the students completed the IDI within a regular school lesson. At the start of their education, they might not have understood the term culture although it is explained within the IDI. At the end of their education, the concentration to complete the IDI may have been affected by students' emotions due to their last day of college with their cohort. However, with identification and description of known biases in the study's sample, biases may be controlled and readers can evaluate biases. A detailed outline of the threats to validity will be provided in Chapter 3 .

\section{Significance}

Diversity is inherent in the Swiss health care as the population demographics change. Several factors contributing to Swiss diversification include an increase in 
foreign-born permanent residents, an increase in a variety of religious beliefs among the population, and, an aging Swiss population (All About Switzerland, 2016; Swiss Confederation, 2018a, 2018b, 2018c/, 2018d). With the increase in the population who are over the age of 65 chronic illnesses have become more prevalent (Obsan, 2008, 2015). Diversity is inherent in the Swiss health care system not only with patients but also health providers (Nguyen \& Mariani, 2014; Swiss Confederation, 2017a/2017b/2018a/ 2018b/2018c; Wurz, 2014). These factors contribute to a changing demographic in the country and a need for health care agencies and providers to be prepared to meet the health services demands.

Although the changing demands on health care providers have increased, the education regulation of health professions in Switzerland has also changed, especially in nursing (OdASanté, 2017; Swiss Education, 2018). These changes have led to a diversification of grades as well as new professional profiles (Institute of Nursing Science at the University of Basel, 2018; Mahrer, 2011; OdaSanté, 2018). Nurses need to be prepared to care for an aging, chronically ill, and diverse population in the health care setting. Nurses with a higher level of intercultural competence In addition to education, health care professionals are also influenced by their own backgrounds and upbringing. Their cultural competence depends on their experiences that influence their perception of diversity and their related behavior such as opinions of their family, friends, and teachers. For example, Swedish and Slovenian health care professionals have exhibited a difference in teamwork, relationships between professions, and responses to patient problems (Pahor \& Rasmussen, 2009). Additionally, Cowan and Norman (2006) reported 
that migrant nurses from other European Union countries employed in London hospitals were perplexed by the diversity of cultures in the United Kingdom. Health care providers are also influenced by their environment such as the current situation regarding refugees from Syria and elsewhere in Europe (“Migration Crisis," 2015).

Nurses with an ethnorelative orientation cultural difference and commonality are able to better deal with cultural diversity than nurses in an ethnocentric stage (Hammer, 2012). An increase of the intercultural competence in nurses contributes to positive social change, as nurses are providing health care service for the society and perform better with higher competencies. Therefore, the nursing students' development of intercultural competence needs to be understood. Accordingly, this quantitative research study was an investigation into the effect on nursing students' orientation toward cultural difference and commonality and its relationship to the cohorts' demographic variables as well as demographic factors of the nursing students.

\section{Contribution to Positive Social Change}

Fear and insecurity, prejudices, and ethnocentrism are elements that are relevant for the understanding of antihuman attitudes. This statement is consistent with the ethnocentric developmental stages of intercultural sensitivity (Bennett, 2011). The way to address these attitudes is education and experience of otherness (Cattacin et al., 2006, p. 7). There are two logics against the risk of increasing radical opinions: information and social relations between different people. The informational path is the focus of many campaigns and educational programs in schools. Efforts to create experiences of difference is easy for people in a favorable position but need to be facilitated for a large 
part of the population (Cattacin et al., 2006). In this perspective, programs of the revitalization of social relations between difference can address attitudes based on prejudices and a lack of experience. However, these relations between different people depends on the intercultural competence of the involved individuals.

As nursing practice is patient centered, nurses providing culturally competent care can "increase access, improve the quality of care, and improve patient satisfaction, leading to better health outcomes for culturally diverse groups" (Gallagher \& Polanin, 2015, p. 334). Therefore, this study may affect positive social change by providing more knowledge to educators who can help increase intercultural competence by understanding contributors to the development of the intercultural competence of nurses.

\section{Summary}

With an aging, chronically ill, and diverse population in the health care setting, diversity in the Swiss health care system is growing. Diversity may lead to marginalization and discrimination, which may be accentuated in individuals who perceive they are different from the majority, not only because of ethnicity and language but also age, gender, or disability. According to the Swiss Red Cross (2018), unreflective generalizations and prejudices by health professionals about individuals or whole groups of people can lead to issues with appropriate treatment and care. Consequently, nurses are required to deal effectively with diversity to provide appropriate care to patient. Intercultural competence is not a personal trait but a competence relying on orientation toward cultural difference and commonality. An ethnorelative orientation toward cultural difference and commonality dealing with diversity is most effective (Hammer et al, 
2003). The development of mindsets needs to be understood better, and further knowledge is needed to contribute to advance practice. Previous research regarding contributors to the development of intercultural competence particularly on nursing is limited. The results of previous research will be presented within the next chapter. 
Chapter 2: Literature Review

Introduction

Diversity is inherent in the Swiss health care as the population demographics change. Several factors contributing to Swiss diversification include an increase in foreign-born permanent residents, an increase in a variety of religious beliefs among the population, and an aging Swiss population (All about Switzerland, 2016; Swiss Confederation, 2018a, 2018b, 2018c, 2018d). With the increase in the population who are over the age of 65, chronic illnesses have become more prevalent (Obsan, 2008, 2015). These factors all contribute to a changing demographic in the country and a need for health care agencies and providers to be prepared to meet the health services demands. With the changing demands on health care providers, education regulation of health professions in Switzerland has also changed, especially in the field of nursing (OdASanté, 2017; Swiss Education, 2018). For example, demands have led to a diversification of grades in nursing as well as to the development of new professional profiles (Institute of Nursing Science at the University of Basel, 2018; Mahrer, 2011; OdaSanté, 2018).

Collaboration is inherent in nursing (Bachmann, 2013; University of Bern, 2014; German Medical Science, 2014), and diversity is inherent in the Swiss health care system (Nguyen \& Mariani, 2014; Swiss Confederation, 2017a, 2017b, 2018a, 2018b, 2018c; Wurz, 2014). Nurses need to be prepared to care for an aging, chronically ill, and diverse population, being culturally and linguistically prepared to meet patients' needs (International Council of Nursing, 2013. Nurses with a higher level of intercultural 
competence have more of an orientation toward cultural difference and commonality in an ethnorelative stage on the IDC, which helps them address cultural diversity (Hammer, 2012). Though nursing students' development of intercultural competence needs to be understood, knowledge is limited. To foster the intercultural competence among nursing students, nursing educators need more knowledge to better understand the contributors to the development of intercultural competence of nursing students. Therefore, this study was conducted to know more about the contributors to intercultural competence, particularly in nursing in Switzerland.

In this chapter, the results of the literature review will be presented. The literature search strategy including key search terms, inclusion/exclusion criteria, and combinations of search terms will be given to provide traceable information to the procedure. The theoretical foundation of this study will be also be described in terms of the origin of the theory, the rationale for the choice of the IDC, and the theoretical propositions. The theoretical propositions will be explained by highlighting the major propositions regarding orientation toward cultural difference and commonality as well as culture, intercultural competence, and diversity including the delineation of related assumptions. The measurement of the key variable orientation toward cultural difference and commonality and the relation between the theoretical foundation and the present study will also be outlined. The literature review related to the key variables will be structured according to the dependent variable orientation toward cultural difference and commonality and the independent variables. A summary and conclusion will be provided at the end of this chapter. 


\section{Literature Search Strategy}

The following library databases were accessed to find relevant articles: Cochrane Database of Systematic Review, CINAHL \& MEDLINE Combined Search, Ovid Nursing Journals Full Text, ProQuest Nursing and Allied Health Sources, ProQuest Dissertation and Thesis Global, PubMed, Thoreau Multi-Database Search. The references of the identified articles were consulted in the process of backward chaining (consulting the references of the article) and to forward chaining (consulting the studies that cite this article) to include all relevant articles including the most recent studies. The following combinations of search terms were used to search the databases: Nursing OR nurse OR nursing student AND/OR intercultural competence OR intercultural OR transcultural OR cultural OR socio-cultural OR diversity OR diverse population OR Intercultural Development Inventory AND/OR simulation OR study abroad OR teaching OR education $\mathrm{OR}$ teaching intervention $\mathrm{OR}$ educational program $\mathrm{OR}$ course. In Table 1 the keywords and phrases as well as the inclusion and exclusion criteria that were applied to find the relevant articles are displayed. 
Table 1

Keywords, Phrases, and Inclusion Criteria/Exclusion Criteria

\begin{tabular}{|c|c|c|}
\hline Keywords \& phrases & Inclusion criteria & Exclusion criteria \\
\hline nurses & $\begin{array}{l}\text { other terms such as nursing, health } \\
\text { professional, health care provider; } \\
\text { all other health care providers such } \\
\text { as physician, physiotherapist, etc. } \\
\text { students within undergraduate or } \\
\text { postgraduate nursing education or } \\
\text { another health care education; }\end{array}$ & $\begin{array}{l}\text { Health care professionals outside } \\
\text { of the scope of clinical practice } \\
\text { or education such as managers, } \\
\text { politicians, etc.; } \\
\text { incoming nursing students }\end{array}$ \\
\hline intercultural competence & $\begin{array}{l}\text { other terms such as orientation } \\
\text { toward cultural difference and } \\
\text { commonality; } \\
\text { other terms in place of intercultural } \\
\text { such as transcultural, }\end{array}$ & $\begin{array}{l}\text { Culture-related to other issues } \\
\text { such as culture of patient safety, } \\
\text { culture of civility, improvement } \\
\text { culture or culture crash } \\
\text { regarding nursing student's }\end{array}$ \\
\hline intercultural competence & $\begin{array}{l}\text { sociocultural, cultural, culture, } \\
\text { diversity, diverse population, } \\
\text { other terms in place of competence } \\
\text { such as competency, } \\
\text { communication, sensibility, } \\
\text { sensitivity, awareness; } \\
\text { name of measurement instrument } \\
\text { Intercultural Development } \\
\text { Inventory or model Intercultural } \\
\text { Development Continuum or } \\
\text { Developmental Model of } \\
\text { Intercultural Sensitivity }\end{array}$ & $\begin{array}{l}\text { experiences of implementation } \\
\text { of evidence-based practice in } \\
\text { clinical practice, etc. }\end{array}$ \\
\hline $\begin{array}{l}\text { contributors to intercultural } \\
\text { competence }\end{array}$ & $\begin{array}{l}\text { any educational intervention } \\
\text { including other terms such as } \\
\text { teaching intervention, teaching } \\
\text { method, education, educational } \\
\text { program, course, simulation, study } \\
\text { abroad } \\
\text { any other contributors regarding the } \\
\text { subjects' demographic variables } \\
\text { such as age, gender, country of } \\
\text { citizenship, ethnic minority group } \\
\text { affiliation, migration background, } \\
\text { world region having primarily lived } \\
\text { up to age } 18 \text {, total amount of time } \\
\text { having lived in another country, } \\
\text { having had lessons about own } \\
\text { culture or any foreign cultures, and } \\
\text { previous education as a health care } \\
\text { assistant }\end{array}$ & $\begin{array}{l}\text { Studies that are applying } \\
\text { interventions based on any other } \\
\text { theoretical framework than the } \\
\text { model Intercultural } \\
\text { Development Continuum or the } \\
\text { Developmental Model of } \\
\text { Intercultural Sensitivity or using } \\
\text { any other measurement } \\
\text { instrument than Intercultural } \\
\text { Development Inventory, apart } \\
\text { from studies providing evidence } \\
\text { regarding contributors related to } \\
\text { the characteristics or experiences } \\
\text { within health care provider. }\end{array}$ \\
\hline
\end{tabular}


Only articles from peer-reviewed journals were included. The period for the publication of the studies was in 15 years (2003-2018). To obtain a fuller picture of the review topic quantitative, qualitative and mixed method studies were included. There are potential biases within this search. The literature search was limited to published studies, which may be selection bias. Articles in another language than English or German were not retrieved, which may be language bias. However, several databases were consulted to prevent publication bias as much as possible.

\section{Theoretical Foundation}

The theoretical foundation for this study was the IDC, which consists of the orientations toward cultural difference and commonality describing sets of knowledge, attitudes, and skills. The orientations toward cultural difference and commonality are arrayed along a continuum from the more monocultural mindsets of denial and polarization through the transitional orientation of minimization to the intercultural or global mindsets of acceptance and adaptation (Hammer, 2012). Figure 5 visualizes this development.

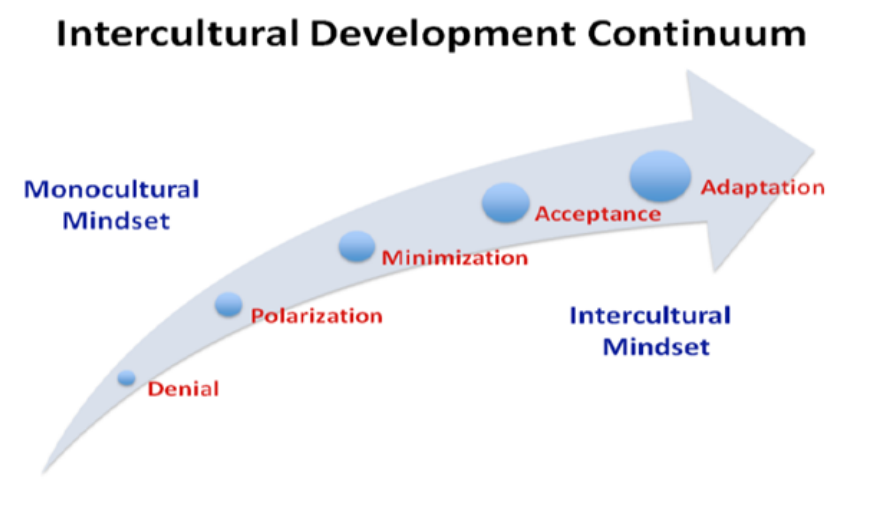

Figure 5. The Intercultural Development Continuum (Hammer, 2012). 


\section{Origin on the Intercultural Development Continuum (IDC)}

The IDC is adapted from the DMIS proposed by Bennett (1986, 1993, 1998, 2001, 2004, 2009, 2011, 2013), who explained how people experience and engage cultural differences in a continuum of experience extending from ethnocentrism to ethnorelativism. Ethnocentrism is individuals' experience of their culture as central to reality, and ethnorelativism is the experience of cultures as relative to context. The developmental applies to anything defined as a cultural difference; it is permanent in one direction, but there may be retreats from some stages (Bennett, 2011).

The DMIS by Bennett (2011) fulfills the criterion that all assertions have been tested through data analysis. The DMIS was created by Bennett over several years of research, revising is between 1986 and 2011 (Bennett, 1986, 1993, 2004, 2013). According to Bennett (2013), his assertions are based on "observations made in both academic and corporate settings about how people become more competent intercultural communicators" (p. 8). He organized these observations into positions along a continuum of increasing sensitivity to cultural difference using concepts from constructivist psychology and communication theory. This is consistent with grounded theory, which researchers use to develop concepts based on observations and analysis (McEwen \& Wills, 2014).

Since the original DMIS was proposed, findings of research using the IDI have both supported the basic tenets of the DMIS and provided a revision of some aspects of its framework (Hammer, 2009, 2011). For example, the IDC by Hammer $(2008,2012)$ represents the revised theoretical framework. The main revisions to the original DMIS 
that are incorporated into the IDC (Hammer, 2012) are related to the minimization identified as a transitional orientation between monocultural and intercultural mindsets and to the integration concerned more with intercultural identity than development. Both revisions will be described in the following paragraphs.

The minimization orientation is identified in the original DMIS formulation as ethnocentric, although IDI research indicates that the minimization orientation is not ethnocentric (i.e., not monocultural). However, minimization is also not ethnorelative (i.e., this mindset is not intercultural/global); its focus on identifying commonalities among diverse groups tends to mask deeper recognition of cultural differences. Thus, minimization is now represented as a transitional orientation between monocultural and intercultural mindsets (Hammer, 2012).

The DMIS identifies denial, defense, reversal, minimization, acceptance, adaptation, and integration as the primary stages of intercultural development. IDI v3 validation confirms denial, polarization (which consists of defense and reversal), minimization, and adaptation as the primary orientations of intercultural competence. Integration, posited in the DMIS as a stage beyond adaptation, is not theoretically related to the development of intercultural competence- the focus of the IDI (Bennett, 2004; Hammer, 2011). Rather, integration as described in the DMIS is concerned with the construction of an intercultural identity rather than the development of intercultural competence (Hammer, 2012). The IDI assesses cultural disengagement, which is the degree to which an individual or group experiences a sense of disconnection or detachment from a primary cultural group (Hammer, 2012, IDI, 2018b). IDI research 
shows that this dimension is conceptually located outside the IDC, is not an orientation or dimension of intercultural competence, and is not a dimension of the integration stage identified in the original DMIS (Hammer, 2012).

\section{Rationale for the Choice of the IDC and the Theoretical Propositions}

A theory is socially significant when it is about "people experiencing a health condition that currently is regarded as having some practical importance by the general public and members of one or more disciplines" (Fawcett \& Garity, 2009, p. 76). Because there is a need to provide health care service in the presence of significant cultural diversity, the IDC by Hammer (2012) based on the DMIS by Bennett (2011) as a framework to explain how people experience and engage cultural difference is socially significant. A theory is theoretically significant when "the information given in the research report about theoretical significance should tell you that the research focuses on the next meaningful step in the development of a theory about people with a certain health condition" (Fawcett \& Garity, 2009, p. 76). The IDC by Hammer provides the knowledge of how cultural difference is being experienced, which is relevant for the experience of people receiving health care and health care providers.

The major theoretical proposition of the IDC (Hammer, 2008, 2012) are the five orientations toward cultural difference and commonality along a developmental continuum. The main concepts incorporated within the orientations toward cultural difference and commonality are culture, diversity, and intercultural competence that will be outlined in detail in the following sections in comparison to the related assumption of other definition of these concepts. This comparison between the theory's assumptions and 
other theories' assumptions will demonstrate the rationale for the choice on the IDC (Hammer, 2012) as the theoretical foundation.

Major proposition orientation toward cultural difference and commonality. Hammer (2012) based the IDC on the DMIS by Bennett (2011), defining the orientation toward cultural difference and commonality as representing the major proposition of the IDC. The five mindsets in the development of intercultural competence represent a person's orientation toward cultural difference and commonality:

Denial of cultural difference. Denial is an orientation that allows for recognition of more observable cultural differences (e.g., food) but may not involve noticing the deeper cultural difference (e.g., conflict resolution styles) and may involve avoidance or withdrawal from cultural differences (IDI, 2018b). According to Bennett (1986, 1993, 1998, 2001, 2004, 2009, 2011, 2013) and Hammer $(2008,2012)$, people in this stage are unaware of the cultural difference and do not perceive them. However, they may perceive differences in broad categories such as "foreigner" or "minority." The constructs available for perceiving personal culture are more complex than those available for other cultures, and people may be mostly disinterested in intercultural communication.

The polarization of cultural difference. Polarization is a judgmental orientation where people view cultural differences as oppositional. This judgement can take the form of defense as an uncritical view of personal cultural values and practices and a critical view toward other cultural values and practices. It can also take the form of reversal as a critical orientation toward personal cultural values and practices and an uncritical view toward other cultural values and practices (IDI, 2018b). According to Bennett (1986, 
1993, 1998, 2001, 2004, 2009, 2011, 2013) and Hammer (2008, 2012), differences between people's selves or the norms of their group are experienced negatively and are perceived in stereotyped and polarized ways. Cultures are organized into $u s$ and them, where typically us is superior, and them is inferior. This point of view can be reversed; however, the point of view is still polarized.

Minimization of cultural difference. Minimization is an orientation that highlights cultural commonality and universal values and principles that may also mask deeper recognition and appreciation of cultural differences (IDI, 2018b). According to Bennett (1986, 1993, 1998, 2001, 2004, 2009, 2011, 2013) and Hammer (2008, 2012), people try to avoid stereotypes and even appreciate differences in language and culture. However, people assume that their own physical or psychological experiences are shared by people in all culture or that certain basic values and beliefs transcend cultural boundaries. The stressing of cross-cultural similarity reduces defense, so people are more tolerant of superficial cultural diversity. People view many of their own values as universal rather than viewing them simply as part of their own ethnicity. Because it includes the masking of dominant culture privilege by a false assumption of equal opportunity, this stage is particularly difficult to pass through.

Acceptance of cultural difference. Acceptance is an orientation where people recognize and appreciate patterns of cultural difference and commonality in their own and others' cultures (IDI, 2018b). According to Bennett (1986, 1993, 1998, 2001, 2004, 2009, 2011, 2013) and Hammer (2008, 2012), people perceive their own culture as just one of some equally complex point of views. They can shift perspective while still 
maintaining our commitment to values. This commitment is important because acceptance does not mean agreement—-the cultural difference may be judged negatively—but the judgment is not ethnocentric. People at acceptance are curious about and respectful toward cultural difference.

Adaptation to cultural difference. An orientation that is capable of shifting cultural perspective and changing behavior in culturally appropriate and authentic ways (IDI, 2018b). According to Bennett (1986, 1993, 1998, 2001, 2004, 2009, 2011, 2013) and Hammer (2008/2012) people can take the perspective of another culture and operate successfully within that culture. They generate appropriate alternative behavior in a different cultural context and therefore enact their intercultural sensitivity as intercultural communication competence. People's knowledge of other cultures in the previous stage did not allow them to adapt their behavior to different cultural contexts easily. Adaptation involves intercultural empathy or experiencing the world to some extent as if people were participating in the different culture.

In summary, the denial and polarization mindsets are monocultural in their orientation and reflect the view that people's "own culture is central to reality" (Bennett, 1993, p. 30). The intercultural/global mindsets of acceptance and adaptation represent a greater capability of shifting perspective and adapting behavior to cultural context. Individuals with an acceptance or adaptation mindset understand that one's own cultural patterns are "not any more central to reality than any other culture" (Bennett, 1993, p. 46). In between the intercultural/global mindsets of acceptance and adaptation and the monocultural perspectives of denial and polarization is the transitional orientation of 
minimization. Minimization is not monocultural in its capability, yet it is also not fully intercultural in its recognition of deeper patterns of cultural difference and the ability to appropriately respond to these differences (Bennett, 2004; Hammer, 2012; Hammer et al., 2003).

While the internal consistency is given, the question whether the propositions of the theory are reasonable needs to be answered. The propositions assume that intercultural competence must be developed within a process over time. While the perceptual organization of cultural difference within people becomes more complex, people's experience of culture becomes more sophisticated and the potential for exercising competence in intercultural relations increases (Intercultural Development Research Institute, 2014). These assumptions derived from observations within people experiencing differences and are therefore inductively reasoned (Fawcett and Garity, 2009). However, if a consulter of Bennett's theory believes that this development occurs in every human being, it would not be adequate, because people do not move automatically from one stage to another. To enhance for instance nurses' cultural competence, it is essential that they engage in cultural encounters and appropriately reflect their experiences with cultural differences (Vande Berg, 2007/2009; Vande Berg, Balkcum, Scheid, \& Whalen, 2004; Vande Berg, Connor-Linton, \& Paige, 2009). If not, it can also happen that people experiencing cultural differences fall back to a previous stage, for instance from early minimization to defense (Bennett, 2011).

\section{Propositions about culture and intercultural competence including}

delineation of related assumptions. The term cultural is used within the theoretical 
proposition orientation toward cultural difference and commonality and the term intercultural competence. It is essential that the same term and same definition is used consistently for each concept. According to Fawcett and Garity (2009), "semantic clarity requires that even concepts that are generally understood in everyday language must be clearly defined when used in theories" (p. 78). While the term intercultural competence has gained considerable currency, naming and defining such a concept is, in fact, no simple matter. Spitzberg and Changnon (2009) cataloged well over 300 terms associated with intercultural competence, illustrating the challenges of clear conceptualization. Within the IDC based on the DMIS, the term cultural is used in every concept about difference and commonality. Bennett (2001) distinguished between objective and subjective culture.

Objective culture. The objective culture relates more to those ethnical aspects such as social, economic, political, and linguistic systems as well as historical influences. For instance, courses in Japanese culture or African American culture are likely to focus on the history, political structure, and arts of the groups. However, Bennett (2001) explained that one can know a lot about the history of a culture and still not be able to communicate with an actual person from that culture. Understanding objective culture may create knowledge, but it does not necessarily generate competence. This statement is consistent with Cerezo, Galceran, Soriano, Camps, and Moral (2014) who focused the lecturers' emphasis on "making the students more aware rather than in acquiring knowledge as it is impossible to know of and understand all about a culture or all the 
cultures" (p. 266). Focusing on the objective culture could promote the risk of the development of stereotypes.

Nevertheless, researchers such as Amerson (2012) relate the term culture without further definition to nationality by referring to "the culture of the host country" (p. 8) when they sent their American senior-level baccalaureate nursing students to an International Service-Learning Experiences to Guatemala and Equador. Also, researchers such as Allen, Brown, Duff, Nesbitt, and Hepner (2013) implicitly refer to an ethnic minority group when talking about culture without defining the concept any further. This reference is consistent with Baghdadi, and Ismaile (2018) who saw the lack of minority nursing educators that represent the ethnic and racial minority groups living in the United States also contributes to the problem of the low levels of nursing faculty cultural competence referring to the National League for Nursing (n. d.). Alpers and Hanssen (2014) differentiated their view from other studies using the concepts of culture and ethnicity as synonyms by stating that "within any cultural or ethnic group one will find considerable diversity; while a person's values, beliefs, and customs may be culture based, they will also be unique to that individual" (p. 1000).

Subjective culture. Emphasizing subjective culture, Bennett (2001) explained that a more useful definition of culture is "the pattern of beliefs, behaviors, and values maintained by groups of interacting people." (p. 3). This definition implies the interaction in any group (subjective culture) in contrast to the social, economic, political, and linguistic systems as well as historical influences referring more to ethnic or national groups (objective culture). An individual may subjectively belong to various groups 
while interacting within those groups in contrast to an objective ethnic or national affiliation. Therefore, the idea of subjective culture gives more direct insight into the worldview of various cultural groups. With the subjective culture, Bennett (2001) sees the key to understanding the junction between domestic and international diversity. As a result, Bennett (2004) derived his theory from this definition of subjective culture and his understanding of international and domestic cultural difference with his background within the American Society. Accordingly, his definition leaves space to interpretations as the terms international and domestic still implies to the affiliation to a nation without dearly define what the domestic culture(s) represents. Domestic culture may refer to gender, age, health condition, profession, social activities, sexual orientation, eating behavior, etc... However, culture refers to more than international or domestic cultural differences and the social affiliation to a specific ethnic group.

Evolution of definition of culture in health care. Culture has been a major construct in anthropology for nearly a century (Rottig \& Reus, 2018). Leininger (1971) first introduced the concept of culture in nursing by emphasizing it as equally as important than care. Leininger (1971) emphasized the powerfulness of the culture care dual construct as the major concept in her Theory of Culture Care Diversity and Universality to discover and understand the illness, wellness, and other human health expressions. This is consistent with Schouler-Ocak, Graef-Calliess, Tarricone, Qureshi, Kastrup, and Bhugra (2015) who discussed the guidelines of the European Psychiatric Association and explained that "the diversity of cultures, ethnicities, races, and reasons for migration poses a challenge for those seeking to understand how illness is 
experienced by immigrants whose backgrounds differ significantly from their clinicians" (p. 431). These authors claimed that the stress of migration as well as social factors and changes related to the receiving society might lead to the manifestation of psychiatric disorders in vulnerable individuals after migration.

Leininger (1971, 1991, 2002a, 2002b, 2006) defined culture as the learned, shared, and transmitted values, beliefs, norms, and lifeways of a particular culture that guide thinking, decisions, and actions in patterned ways. Leininger intended with her theory that the nursing profession should discover differences and commonalities in culture care through naturalistic inquiry. She saw the cultural influences on caring within technological, religious and philosophical, social, economic, and educational factors. This view is consistent with the Bennett's (2001) definition of subjective culture. Hoverer, the goal of the Leininger's theory was to develop knowledge that is culturally specific and congruent so that appropriate care can be given. Therefore, Leininger's definition of culture may be similar to Bennett's (2001), but the intention of how to deal with it is different.

Evolution of various definitions of intercultural competence. Andrews and Boyle (2002) expanded on Leininger's work by designing a model for transcultural assessment in clinical and community contexts. Areas to assess included values orientation, health-related beliefs and practices, communication patterns, cultural sanctions, socioeconomic considerations, religion, and others. Similarly, Giger and Davidhizar (2002) proposed the transcultural assessment model, which conceptualized the client in unique cultural heritage. Knowledge of communication patterns, time and 
space orientations, environmental control, social organization, and biologic variations guide the health care provider in delivering culturally competent care. Giger and Davidhizar's (2002) provide with their model a guide to cultural assessment. Also, Purnell's $(2000,2002)$ model for cultural competence includes 12 domains of culture as they relate to individuals, families, communities and global society to be used as a guide of cultural assessment of a patient. However, they still refer to the importance of knowing a certain culture to provide the appropriate care. For example, Andrews and Boyle (2002) stated that "cultural assessment skills, combined with the nurse's critical thinking ability, will provide the necessary knowledge on which to base transcultural nursing care" (p. 179). However, they admitted that "memorizing the esoteric health beliefs and practices of a litany of different groups" (Andrews \& Boyle, 2002, p. 179) is not effective given that nurses are likely to encounter people from literally hundreds of different cultures and suggested to acquire the knowledge from all cultural groups that they encounter to assess and care for diverse patients. Furthermore, some scholars also emphasized that knowledge explicitly intend to promote interculturality risks reinforcing the hold of received Western, colonial ideas regarding the other and what needs to be done to correct the other's development deficits (Stein, Andreotti, Bruce, \& Susa, 2016; Terzuolo, 2018). Kumas-Tan, Beagan, Loppie, MacLeod, and Frank (2007) highlighted some of the problematic, unexamined assumptions about cultural competence that are embedded in the most widely used cultural competence measures. They stated that even when concerns beyond race and ethnicity are considered (which is rare), this view of culture "assumes that the locus of normalcy is white, Western culture - that difference means 
nonwhite, non-Western, non-hetero sexual, non-English-speaking, and most recently, non-Christian -how they are different from us" (p. 555). About such considerations, the need for caution in using knowledge acquisition is essential.

Although Purnell's (2002) and Campinha-Bacote's (2002) model emphasized the cultural competence as a process rather than a result, the acquirement of particular cultural knowledge is still emphasized. Campinha-Bacote (2002) incorporated the concepts of cultural awareness, cultural desire, cultural knowledge, cultural skills, and cultural encounters in her model The Process of Cultural Competence in the Delivery of Healthcare Services. Campinha-Bacote (2002) emphasized the cultural knowledge as the "process of seeking and obtaining a sound educational base about culturally and ethnically diverse groups." (p. 13). Also, cultural skill is related to "the ability to collect culturally relevant data regarding the patient's presenting problem, as well as accurately performing a culturally-based physical assessment in a culturally sensitive e manner" (p. 13). Campinha-Bacote (2002) claimed that this process involves learning the skills of how to conduct a cultural assessment, an essential task in delivering patient-centered care. In summary, the theorists emphasizing the cultural assessment did not consider that knowledge alone does not assure that the nurse transfers this knowledge into appropriate care. Such a view does not consider the nurse's worldview and how she perceives any differences between the patient's and her own worldview and how this influences her attitude toward the patient.

The Panel of Experts on the Cultural competence of the American Nursing Association (Giger et al., 2007) agrees that a health care professional has acquired 
cultural competence when they are aware of their own thoughts, sensations, and feelings and they do not allow these factors to influence the care they give. Rather they incorporate their respect for the patient's culture into providing better care. This statement is consistent with Campinha-Bacote (2002) who highlighted that both appreciation and respect are based on feeling comfortable within this cultural diversity instead of false flattery to other cultures. Cai (2016) who conducted a concept analysis of cultural competence reported that respect is implied within the term cultural sensitivity referring to nurses' appreciation, respect, and comfort to the cultural diversity of patients. Because the culture of every individual cannot be assumed to be the same, and cultural diversity is inevitable, this diversity should be appreciated to achieve mutual learning for common progress (Cai, 2016). Moreover, the idea that one's own culture is superior to another's should be avoided (Dudas, 2012; Giger et al., 2007).

Ethnocentric versus ethnorelative orientation toward culture. Models of cultural competency such as the model of Leininger (1971, 1991, 2002, 2006), Andrews and Boyle (2002), Purnell (2002), or Campinha-Bacote (2002) may be criticized for "referring to cultural groups in terms of ethnicity, resulting in a narrow, essentialist and limiting view of culture, as opposed to the more fluid constructionist view" (Albarran, Rosser, Bach, Uhrenfeldt, Lundberg, \& Law, 2011, p. 2). The view that patients are the others in opposition to the nurses emphasizes foreignness. It implies the focus on patients being (for example ethnically) different compared to the (ethnic) majority of the nurses. This includes a view that one culture is superior to another one. 
During the 1960's and 1970's, when racial discrimination was declared illegal in the United States, it was considered a fair and liberal way of treating others and saying, "I treat everyone the same." was appropriate. This attitude "assumes that sameness equals fairness, an assumption that only holds true if the values and norms of people involved in an interaction are similar" (Elliott, Adams, \& Sockalingam, 2018, para. 1). Within the IDC by Hammer (2012) such a speaker would be in the stage of minimization. However, in the ' 80 's and '90's organizations went beyond discrimination issues and even celebrated diversity. According to Elliott at al. (2018) celebration of diversity falls far short of what is needed for effective intercultural collaboration. To move beyond celebration to a real ability to work appropriately with cultural difference and commonality requires more knowledge about intercultural competence.

Bennett (2011) and Hammer (2012) developed the knowledge about intercultural competence on a next level. Bennett (2011) and Hammer (2012) do not mainly focus on culture care, meaning to provide the right care to a particular culture unlike previous assumptions such Leininger's theory of culture care stating that "every human culture has folk remedies, professional knowledge, and professional care practices that vary. The nurse must identify and address specific folk remedies consciously with each client to provide holistic and culturally congruent care” (Sitzman \& Eichelberger, 2011, p. 96). Bennett (2011) and Hammer (2012) assumed that cultures are highly differentiated and that there are various ways in which people can understand and organize their relationship with the external environment in which they live. This assumption includes but is not limited to social, political, economic and religious affiliations. According to 
Hammer et al. (2003), "the crux of the development of intercultural sensitivity is attaining the ability to construe (and thus to experience) cultural difference in more complex ways." (p. 423). Bennett (1993) explained that this ability is developed through experience; it is not an innate perspective. The underlying assumption of Bennett's (1993) model is that competence in dealing with intercultural relations increases as one's understanding of cultural difference becomes more sophisticated as one's worldview incorporates cultural differences into a new identity. Therefore, both authors focus more on the experience of cultural differences. Within the first three stages one sees his own culture as central to reality, the point of view is ethnocentric. Within the next three stages of the further development, one experiences one's own culture as in the context of other cultures, the point of view is becoming more and more ethnorelative. In summary, the theory is socially and theoretically significant, as it provides a theoretical framework for further studies as well as the focus on one's next meaningful step in one's development of intercultural competence. Therefore, the contributors to the intercultural competence within nurses can be based effectively upon this theory as a theoretical framework.

Selected definition of culture and intercultural competence. Hammer et al. (2003) used the term intercultural sensitivity to refer to the ability to discriminate and experience the relevant cultural difference, and the term intercultural competence to mean the ability to think and act in interculturally appropriate ways. Hammer et al. (2003) argued that greater intercultural sensitivity is associated with a greater potential for exercising intercultural competence. Intercultural competence "reflects the degree to which cultural difference and commonality in values, expectations, beliefs, and practices 
are effectively bridged, an inclusive environment is achieved, and specific differences that exist in one's organization are addressed from a mutual adaptation perspective" (IDI, 2018b, p. 3). The capability of deeply shifting cultural perspective and bridging behavior across cultural differences is most fully achieved when one maintains an Adaptation perspective (Hammer, 2012).

Although, Bennett (2011) did not explicitly explain how the terms intercultural sensitivity and intercultural competence must be seen in contrast to the same term used by other authors such as within the Campinha-Bacote (2002), it is distinguishable from the development from ethnocentric to the ethnorelative emphasizing that no culture or worldview is superior to any other. The view that no culture or worldview is superior to any other is what is incorporated in the models by Bennett (2011) and Hammer (2012) by emphasizing ethnorelative views. Hammer (2012) comprehensively explained that ethnorelative orientation toward cultural difference and commonality enables to deal more effectively with cultural difference and commonality. Accordingly, Hammer (2012) defined the intercultural competence as "the capability to accurately understand and adapt behavior to cultural difference and commonality" (IDI, 2018b, p. 3). Therefore, Hammer's (2012) definition of intercultural competence implying Bennett's (2001) definition of subjective culture Bennett (2001) as "the pattern of beliefs, behaviors, and values maintained by groups of interacting people." (p. 3) is included in this study, because it is coherent in dealing more effectively with diversity.

\section{Propositions about diversity including delineation of related assumptions.}

According to Merriam Webster's Learning Dictionary (2018) diversity is defined as "the 
condition of having or being composed of differing elements" and as a "variety, especially the inclusion of different types of people (as people of different races or cultures) in a group or organization." These definitions refer to terms such as difference and variety. Furthermore, they relate to the term compose/composition, meaning a kind of mixture. The synonyms are terms like diversification, assortment, dissimilarity distinction, distinctiveness, divergence, diverseness, heterogeneity, medley, multiplicity, range, unlikeness, variance, variousness (Thesaurus.com, 2018). Consequently, the antonyms of diversity are terms such as agreement, sameness, identicalness, uniformity, and similarity (Thesaurus.com, 2018). The term diversity is used in the literature within various other disciplines such as biology (biological diversity) or technology respectively engineering (diversification of technological products and processes). In most disciplines, the term diversity appears about social issues. For this research, the focus to define diversity was narrowed to the discipline of nursing including nursing education and related literature has been consulted.

The term diversity used in nursing research. The authors of articles often do not clearly define what they mean by the term diversity. For example, an article identified explicitly about diversity within nursing and education is about diversity barriers and perceptions of recruitment, hiring, and retention within nursing faculty diversity by Salvucci and Lawless (2016). However, they used the term diversity thirteen times without defining this term once. Not so, Koch, Philips and Davidson (2015) who explained that diversity is concerned with the difference between people traditionally classified based on visible versus non-visible difference, and surface versus deep 
difference. Koch et al. (2015) stated that these simplistic classifications do not consider that individuals may have numerous differences or diversity characteristics making diversity a multifaceted and complex concept with many nuances. For their literature review, Koch et al. (2015) defined diversity broadly including age, gender, ethnicity, language, religious belief, sexual orientation, educational background, income, marital status, parental status, and work experience and disability. This definition is consistent with Garneau and Pepin (2015) who provided a constructivist theoretical proposition of cultural competence development in nursing. They claimed that "a culturally diverse context thus encompasses diversity that can assume many forms in society - such as age, gender, sexual orientation or socioeconomic status - and is not limited to race and ethnicity" (p. 1062). Another author investigating more deeply within the meaning of diversity within nursing education is Leonard (2006). She advocated that schools of nursing address the issue of diversity in their curricula and overall programs, because of the current and future demographics of her country, the United States. Accordingly, she conducted a literature review on the term diversity.

A broad definition of the term diversity. Leonard (2006) reported that no precise definition of diversity emerged from the data, but the concept of difference is implied by the context in which certain terms describing difference among people are used. Leonard (2006) conducted a qualitative content analysis. The units of observation were self-study documents written by schools of nursing seeking accreditation from the National League for Nursing Accrediting Commission. From the data, it could be deduced that the category of people different than us consists of people who do not fit the characteristics 
or roles of what an institution might perceive as typical for their student bodies or faculties. This deduction is consistent with the synonyms and antonyms of the term diversity mentioned above (Thesaurus.com, 2018). According to Leonard (2006), the literature did not explicitly state or define what constitutes a typical characteristic versus an atypical characteristic. However, the fact that certain characteristics were singled out in the documents as worthy of special consideration or special treatment suggests the concept of difference. Table 2 outlines the seven sub-themes as discussed by Leonard (2006). 
Table 2

Seven Subthemes of Diversity by Leonard (2006)

\begin{tabular}{|c|c|}
\hline $\begin{array}{l}\text { Subthemes of } \\
\text { diversity }\end{array}$ & Descriptive terms \\
\hline $\begin{array}{l}\text { Race, Ethnicity, } \\
\text { Culture }\end{array}$ & $\begin{array}{l}\text { There were no distinct differences in the usage of the terms race, ethnicity, and } \\
\text { culture in the documents. In fact, in many instances, the terms were used } \\
\text { synonymously. }\end{array}$ \\
\hline Lifestyle & $\begin{array}{l}\text { This sub-theme contains miscellaneous life choices and living circumstances that } \\
\text { may set people apart from the mainstream in colleges and universities. This } \\
\text { category consists of persons who have chosen a particular lifestyle (e.g., sexual } \\
\text { preference) and those whose geographical situations dictate a lifestyle different } \\
\text { from the typical college student. Two other lifestyle groups identified by the data } \\
\text { and supported by the literature are persons who reside in both rural areas and inner } \\
\text { cities. According to one institution, persons living in rural areas have poorer health } \\
\text { and less access to health care than those living in municipal or urban areas. }\end{array}$ \\
\hline Religion & $\begin{array}{l}\text { Most of the colleges and universities participating in the study have either non- } \\
\text { discriminatory policies which prohibit discrimination by religion or creed, or they } \\
\text { have statements which reflect a valuing of religious diversity. }\end{array}$ \\
\hline Circumstances & $\begin{array}{l}\text { There are a variety of life circumstances that may set students apart from the } \\
\text { majority of university students. These circumstances may include but are not } \\
\text { restricted to; any condition is surrounding a person that places him or her in a } \\
\text { situation that may be interpreted as different from the norm. These circumstances } \\
\text { include a person's marital or parental status; }\end{array}$ \\
\hline Circumstances & $\begin{array}{l}\text { residential status; educational background; family background; employment } \\
\text { status; or non-traditional age status. There were } 20 \text { different categories of } \\
\text { circumstances identified by the institutions. The most commonly cited } \\
\text { circumstance was age. }\end{array}$ \\
\hline $\begin{array}{l}\text { National Origin, } \\
\text { Citizenship }\end{array}$ & $\begin{array}{l}\text { This category consisted, of students who were either born in or are citizens of } \\
\text { a country other than the United States. Most of the participating colleges and } \\
\text { universities acknowledge that international students are a source of campus } \\
\text { diversity. }\end{array}$ \\
\hline $\begin{array}{l}\text { Linguistic } \\
\text { diversity }\end{array}$ & $\begin{array}{l}\text { This sub-theme included individuals who do not speak standard English as } \\
\text { their native tongue. Most of the people for whom English was a second } \\
\text { language were from foreign countries. }\end{array}$ \\
\hline $\begin{array}{l}\text { Health Status, } \\
\text { Disabilities }\end{array}$ & $\begin{array}{l}\text { This sub-theme described those students who have health deviations or } \\
\text { impaired physical abilities. }\end{array}$ \\
\hline
\end{tabular}


Selected definition of the term diversity. Although Bennett (2013) used the term cultural difference without clearly defining the term difference semantically, Hammer (2012) used the terms nationality, race, ethnicity, age, family background, abilities/disabilities, religion, educational background, home/geographic roots, sexual orientation, socio-economic status, and work experience within the intercultural development plan. The terms belong to a checklist to reflect on one's experiences with culturally diverse groups regarding the aspect of diversity that have influenced one's views of cultural commonalities and differences (IDI v. 3).

\section{Measurement of the Orientation Toward Cultural Difference and Commonality}

Each concept can be measured, as the developmental stage of the development of intercultural sensitivity can be measured by the IDI (2018a, 2018b). Based on Bennett's theory it was developed and tested by Hammer et al. (2003). According to them, the IDI is the premier, cross-cultural assessment of intercultural competence that is used by thousands of individuals and organizations to build intercultural competence to achieve international and domestic diversity and inclusion goals and outcomes. Therefore, this theory is broadly empirically tested (Fawcett \& Garity, 2009). However, as with every assessment it has to be considered that perceptual strategies may span several stages. Although, one stage is predominant, as the particular configuration of perceptual strategies used by each individual and group is their predominant experience of difference. 


\section{The Relation Between the Theory and the Present Study}

The IDC provides a theoretical framework for the development of the intercultural competence. Although the theory does not provide concrete interventions to generally apply within the practice, it provides indications of what each individual needs in which stage of development to stimulate his or her process of becoming more interculturally competent. Hammer (2012) based on Bennett (2011) advises how learners (e. g. nurses) can be challenged regarding the contents and the process and how they can be supported within their development to further stages. Therefore, the IDC is generally appropriate to address such a practice problem such as to foster nursing students to deal appropriately with diverse patients.

\section{Literature Review Related to the Key Variables}

Twelve studies within health care using the Intercultural Inventory (IDI) and published within the last fifteen years have been identified: Altshuler et al. (2003), Boggis (2012), Bourjolly et al. (2015), Halm and Wilgus (2013), Halm et al. (2012), Hamre (2012), Harder (2018), Huckabee and Matkin (2012), Kruse et al. (2014), Larson (2011), Munoz et al. (2009), and Peying et al. (2012). The studies will be presented chronically. As the dependent variable of this study is the nurses' orientation toward cultural difference and commonality measured using the IDI (2018a/2018b) the results of the studies will be pointed out. In addition, the findings of any researched relations between the orientation toward cultural difference and commonality and the participants' demographic variables as dependent variables of this study will be presented. 


\section{Dependent Variable Orientation Toward Cultural Difference and Commonality}

Altshuler, Sussmann, and Kachur (2003) investigated within 24 pediatric resident trainees at a teaching hospital in an urban setting (Brooklyn, New York) in the academic year 2000-2001 by assessing their stage of intercultural sensitivity using the IDI before and after their participation in an intercultural training intervention. The aims of their research were (1) to provide normative data for the IDI on a new population; (2) to assess the predictive value of demographic variables on intercultural sensitivity; and (3) to explore the efficacy of the IDI in gauging the effectiveness of an intercultural sensitivity training program for pediatric residents. The researchers aligned the participants into three groups, workshop and culture OSCE (objective structured controlled evaluation), culture OSCE only, and control. A 2-h workshop provided opportunities to extrapolate culturally different health attitudes and concomitant behaviors from cultural backgrounds of patients by using a comparative values-based methodology linking culture, values, attitudes and beliefs and Hofstede's four cultural dimensions of individualism, power distance, masculinity, and uncertainty avoidance (Hofstede, 1980) as conceptual frameworks for understanding cultural differences. The workshop utilized presentations, critical incidents, group discussion, and self-reflection to increase cultural awareness. The Culture OSCE consisted of four interactive stations, where the residents received a brief overview of the clinical situation and interviewed or counseled actors who had been trained to serve as standardized patients (SP).

The group mean IDI ratings for study participants $(N=24)$ before intervention were as follows: Denial, $M=2.09, S D=0.85$; defense, $M=2.48, S D=1.05$; 
minimization, $M=4.5, S D=1.08$; acceptance, $M=5.44, S D=0.84$; cognitive adaptation, $M=4.77, S D=0.98$; behavioral adaptation, $M=4.69, S D=0.68$. Altshuler et al. (2003) pointed out that the minimization factor was surprisingly high and described the health care professionals as transitioning from ethnocentricism to ethnorelativism. The post-training IDI scores were compared with pre-training scores separately for each of the intervention groups to assess the effectiveness of training on intercultural sensitivity. There were no significant differences between the pre- and post-training IDI scores for either group. The perceived orientation (PO) was 4.96, and the developmental orientation (DO) was 3.88. The gap score of 1.08 reflects a meaningful difference between the group's view of themselves as substantially more interculturally competent than the lower developmental worldview score would suggest (overestimation). Altshuler et al. (2003) found that gender and multicultural experiences can influence one's level of intercultural sensitivity as the results showed that the female residents exhibited lower denial and defense and higher acceptance, cognitive adaptation, and behavioral adaptation compared to their pre-training scores. Males, however, showed an increase in denial and defense and a decrease in acceptance, cognitive adaptation, and behavioral adaptation.

Bourjolly, Sands, Solomon, Stanhope, Pernell-Arnold, and Finley (2005) conducted a study within mental health care to explore the impact of an intensive training program on intercultural competency (PRIME training) over time by using the IDI and writings. PRIME is a statewide intensive training course in Pennsylvania, which aims to reduce culturally based disparities in the provision of mental health services. The 32 
participating mental health providers were required to write reflection papers (logs) on their cultural experiences between sessions and to submit them at the next training session. Bourjolly et al. identified patterns within the trainees' logs and reported that 28 participants moved from ethnocentric to ethnorelative positions, two remained ethnocentric, and four remained ethnorelative. However, they did not provide any score of the IDI. Therefore, the participants' movement is more related to their PO as to their developmental orientation. As it is known from other studies that overestimation of one's intercultural competence is usual, this result needs to be considered carefully.

However, this was the first documented attempt to examine the developmental process using the logs from trainees in cultural competency training over a period of several months. Whereas other studies used pre- and post-test assessments, Bourjolly et al. were able to observe this process qualitatively for each individual monthly, over a period of 10 months. An examination of logs of those trainees who stayed in the ethnorelative stages was qualitatively different from those trainees who remained in the ethnocentric stages, as well as other PRIME trainees who went back and forth between ethnocentric and ethnorelative stages. This difference had to do with the way they described their cultural experiences in their personal and professional lives. It was apparent that those individuals who remained ethnorelative had begun a journey of selfexploration of their culture or those of family members before the PRIME training. They lived bicultural or multicultural lives and gained a great deal of insight from the struggles they have had and currently face from cultural encounters. 
Munoz, DoBroka, and Mohammad (2009) developed a multidisciplinary pilot course for students in the human service professions of nursing, education, and social work at a private midwestern university (Columbus, Ohio) to foster the students' intercultural competence. The course covered the five components of Campinha-Bacote's (2002) process of cultural competence of cultural awareness, cultural knowledge, cultural skills, cultural encounters, and cultural desire. The researchers designed this multidisciplinary course in cultural competence as a seven-week, early evening pilot seminar that met for two hours per week, becoming a forum for discussion and reflection on clinical experience. Further learning included assigned readings and written response papers, role-playing, interacting with guest speakers. Munoz et al. did not report how many students participated in the course, although they stated as a limitation that recruiting students was a challenge as this course was an elective for students in professional programs who already had full schedules with their clinical placements. The authors determined that the IDI was not an appropriate instrument to use to demonstrate short-term, pretest, and posttest gains in their particular course. Therefore, they administered the IDI during week two only to provide a single snapshot of students' collective, developing intercultural competence at this point in their university program. Munoz et al. did not report the results of the IDI but reported that this composite information about students' developmental level of intercultural competence provided the professors with key information that informed the content of lectures, class discussions and reflections, and planned learning opportunities for the remainder of the course. According to the results of a three-question narrative course evaluation, Munoz et al. 
(2009) concluded that this kind of multidisciplinary approach to teaching and learning had been shown to be a benefit to a small sample of students. Students' written reflections indicated growth in the acquisition of cultural knowledge, skills, and desire. Faculty benefited as well, with positive outcomes such as participation in a rewarding, learner-centered teaching collaboration with colleagues who shared common goals in achieving the university's mission.

Larson (2011) conducted a mixed method study to understand the current environment of the intercultural sensitivity of nursing faculty and their ability to meet the needs of cross-cultural nursing students. They investigated within fifty-four nursing faculty (female $=53$ and male $=1)$ from across seven geographic regions across the United States. The response to the research question of, "Using a valid and reliable tool for measuring intercultural sensitivity, where do nursing faculty fall on the continuum?" the majority of faculty were represented placement on the IDI continuum with an aggregate result of a PO in the middle range of acceptance and a DO midpoint in minimization. The leading orientation for the group of 54 participants was identified as residing in minimization. This finding needs to be interpreted to mean that the majority of faculty perceive themselves to be farther along the intercultural sensitivity developmental continuum than they are. With a leading orientation of minimization, it is understood the nursing faculty is minimizing the cultural differences while they perceive themselves to be adapting and accepting of differences. This finding and its interpretation is consistent with the reported overestimation of other studies' participants. 
Hamre (2012) conducted a study with a mixed method approach to identify the process that nursing students go through as they become more culturally competent and to determine if Bennett's (1993) DMIS would facilitate 29 baccalaureate nursing students' becoming more culturally sensitive. She conducted her study at the University of Minnesota. Hamre reported the statistical results of the IDI in detail. Overall the 54 participants represent placement on the IDI continuum with an aggregate result of a PO in the middle range of Acceptance and a developmental orientation mid-point in minimization. Also this result is consistent with the reported overestimation of other studies' participants.

Peiying, Goddard, Gribble, and Pickard (2012) investigated within 16 final-year health professions students of the Curtin University of Technology in Perth, Australia who had successfully applied for the 4-week international placement in China or India. Peying et al. used the students' journal as qualitative data to obtain a rounded understanding of the international cross-cultural experience. The journals allowed participants to document experiences, feelings, and perceptions. The authors highlighted that reflection enabled the students to make sense of their experience, facilitating learning and development. The quantitative data were obtained through pre/post placement testing using the IDI. The results showed the IDI overall score increased from pre- to postplacement within the minimization stage. However, results of the paired sample $t$ test show the change in overall score $(p=.10)$ and individual scale scores are not statistically significant except for the acceptance/adaptation scale $(p=.03)$. The nonsignificant finding could be attributed to the small sample size of sixteen. An influence causing an 
unmeasured yet recognized regression into ethnocentrism before advancing through minimization by the end of placement may have been the initial arrival in the host countries as a response to the culture shock that can never be completely anticipated. Peying et al. identified the five themes (a) increased vigilance and adaptation to the environment, (b) uncertainty and anticipation, (c) grappling with supremacy, (d) recognizing and appreciating differences, and (e) cultural immersion and development from their qualitative analysis suggesting the development of cultural sensitivity. As the qualitative data reveals the often overestimate PO toward cultural difference and commonality of the students Peying et al.'s (2012) conclusion that international placements increase the cultural sensitivity and competency of health professions students may be considered carefully.

Boggis (2012) wanted to examine quantitatively the effectiveness of a curricular program guided by the IDC as measured by the IDI. The participants were seven-teen occupational therapy students of the School of Occupational Therapy, Pacific University in Hillsboro, Oregon as the intervention group and twenty-five non-OT health professional students at a private nonprofit university in the USA as the control group. The characteristics of the two groups were matched. Boggis administered the IDI within the participating students upon matriculation into their respective programs of graduate study and again upon completion of 3 years of study. The occupational therapy students participated in a cultural curricular design guided by the IDC, while the control group participated in the cultural study not guided by the IDC. Though occupational therapy students did not show a significant change in overall developmental orientation mean 
scores from pretest to posttest $(t=0.847, p=.41)$, the results indicate that the designed intercultural curriculum increased intercultural competence among those occupational therapy students who began their program with the monocultural mindset of polarization and moved to the interculturally transitional mindset of minimization. The control group showed a significant decrease in developmental orientation mean scores at posttest $(t=$ $6.1, p<.001)$. No significant group or group by baseline interaction effects were found when comparing overall post developmental scores adjusting for baseline $(F=2.4, p=$ .13). Boggis concluded that the curriculum design as guided by the IDC appears to have mitigated a decrease in competence, although it did not significantly increase the overall cultural competency of occupational therapy students. Results suggest that the cultural challenges that students face appear to be considerable and, without targeted, integrated intercultural preparation, can overwhelm new health professionals’ intercultural capability.

Halm, Evans, Wittenberg, and Wilgus (2012) intended to expand the awareness and comfort of interprofessional team members in caring for patients and families with diverse cultural beliefs and practices at the end of life to improve the patient experience. The study setting was a 34-bed combined medical-surgical oncology unit of a 454-bed regional community hospital in Salem, Oregon. Halm et al. investigated within twentyfour interprofessional team members from nursing, medicine, social work, chaplaincy, care management, respiratory care, pharmacy, and volunteer services practicing on a combined medical-surgical oncology unit. The participants attended an in-service session on the end-of-life care beliefs, practices, and preferences of the Latino, Russian, and 
Micronesian cultures and then participated in critical reflection sessions where culturally specific end-of-life care cases were discussed using a structured dialogue guide. Collectively, the IDI showed that the team's perceived cultural competence was at the level of Acceptance whereas team's developmental orientation was minimization, meaning that the team overestimated its cultural competence. The $t$ tests showed no significant differences between pre-post attitude and knowledge scores $(p>.05)$. However, a baseline, only $4.2 \%$ of interprofessional team members perceived that their developmental orientation was at a level of acceptance and $16.7 \%$ were on the cusp of acceptance. After the educational interventions, $15.8 \%$ of interprofessional team members were at a developmental orientation of acceptance, suggesting that those who were on the cusp of acceptance moved to a higher level along the Intercultural Competence Continuum. Accordingly, Halm et al. concluded that staffs' perceived level of understanding of end-of-life care beliefs, preferences, and practices of the Latino, Russian, and Micronesian cultures, as well as comfort and effectiveness in providing culturally sensitive end-of-life care, were higher after the in-service and critical reflection sessions $(p<.05)$.

Huckabee and Matkin (2012) conducted a study to determine what, if any, improvements in cultural competency were measurable in ninety-three physician assistant (PA) students from four successive classes graduating from the private University of Nebraska between 2003 and 2007. All students were enrolled in specific didactic studies and clinical experiences in cultural sensitivity and competency. The researchers used the Multicultural Awareness, Knowledge and Skills Survey-Revised (MAKSS-R, D’Andrea, 
Daniels, \& Heck, 1991) as a pretest upon program when students matriculated, and again as a posttest on the final day of the program (over a five-year period). The IDI was added in the final year of the study as a second survey to explore further the outcomes measured by the MAKSS-R. The most recent graduating class showed the highest scores (group mean 3.58 on a scale of 1-5) in the minimization developmental stage, while they overestimated their level of intercultural competence. This result places the group in the mid-range of monoculturalism, more highlighting cultural commonality. Huckabee and Matkin claimed that students in this developmental stage of cultural competency would benefit from training that supports the individual's cultural distinctions while promoting skills of integration with other cultures. Therefore, the researchers recommended enhancing curricular instruction such as exploring cultural assessment methods and controversies in health care differences, combined with increased clinical experiences with diverse cultures to help move students past the minimization stage to gain greater cultural competency.

Halm and Wilgus (2013) conducted their research at the Family Birth Center at a 454-bed regional medical center in the Mid-Willamette Valley, Oregon, by using a prepost-evaluation design to evaluate the impact of the cultural competence program. The participants were eighty nursing staff who participated in a cultural competence training program infused with Latino culture, including Spanish language/music, to highlight critical reflections, cross-cultural practice, and communication standards for culturally competent care. Perceptions of cultural sensitivity were evaluated via patient interviews, the IDI, and focus groups. The results of the IDI demonstrated that minimization was the 
group developmental orientation at both time periods. The group's orientation gap between perceived and developmental orientation was greater than seven points. This means the leading orientation for both time periods was acceptance through Adaptation; a range focused on increasing cultural self-awareness by learning culture-general and culture-specific frameworks to more deeply understand patterns of difference that emerge in interactions with people from other cultures. Because of this leading orientation, Halm and Wilgus identified the group as well positioned to look for ways to shift cultural perspective and adapt behavior around cultural differences as its members begin to recognize and appreciate cultural differences more fully. The authors reported that the only secondary orientation, reversal trailing orientation, was seen after the cultural competence training program, indicating that cultural differences were viewed by the group regarding $u s$ and them, including an overly critical view of one's own cultural values and practices and an uncritical view of other cultural values and practices. The researchers concluded that while the cultural competence intervention did not significantly change the intercultural development level of the staff, it did increase staff knowledge, awareness, and understanding of how to effectively serve Latina patients by attending to cultural norms, customs, and values during the birthing experience. The authors argued that "significant change in IDI scores along the cultural competence continuum likely takes a longer period, involving sustained focus and critical reflection" (p. 11). Halm and Wilgus reported the patients' satisfaction with care while many Latina mothers reported situations in which care could have been more culturally sensitive. Therefore, Halm and Wilgus identified their project's key message that it is important 
that health care provider pay close attention to what patients and families say about their satisfaction, because health care provider often overestimate their level of cultural competence resulting in a disparity of the perception between the health care provider and the patient.

Kruse, Didion, and Perzynski (2014) conducted a quantitative study with the purpose to identify the cultural competency of students, faculty, and staff at the College of Nursing of the Lourdes University in Sylvania, Ohio. The sample for this study included 314 participants (students, faculty, and staff). The researchers used the IDI, and most participants scored at the minimization level, similar for students and faculty/staff. Ninety-eight percent of student participants overestimated their level of cultural competency. Minority students had higher cultural competency scores in terms of developmental orientation $(M=98.85, S D=14.21)$ compared to non-minority students $(M=94.46, S D=14.96)$.

Harder (2018) examined the addition of videos to complement a manikin-based simulation experience to improve intercultural competence in undergraduate nursing students within their third year of education at the University of Manitoba, Winnipeg, Canada (the author did not report the sample size). Harder (2018) applied the IDI. The PO score for the group was 122.07. The DO score preintervention for the group was 89.73 (13.45), and the DO score postintervention was 93.11 (17.44). Although there was a 3.38 gain in the score, both prescore and postscore place the groups' development orientation within the minimization range. The total gap score between the PO and DO 
for this group was 30.68 points preintervention and 28.96 points postintervention while a gap score of 7 points or more is a meaningful difference in the interpretation of the IDI.

In summary, the students overestimated their level of intercultural competence, which is not unique. Overestimation has been observed in other studies with health profession students. Although the average score increased slightly in the DO, it did not increase enough to change the stage of the group on the intercultural continuum. While the findings of the empirical studies reporting the contributors to the proband's development of intercultural competence by using the IDI are similar, the studies vary regarding intervention, duration, design, and scope.

The interventions of the studies in relation to the variable exchange program in the last year of the nursing students' education. Mainly, the studies show the heterogeneity of the interventions. The researchers applied the following interventions to foster the intercultural competence of the participants: a) various educational training programs in a classroom (Altshuler et al. 2003, Bourjolly et al., 2005, Halm \& Wilgus, 2013) and experimental learning such as b) in-service session on the end-of-life care Halm et al., 2012), c) video and simulation (Harder, 2018) and d) 4-week international placement (Peying et al., 2012). Huckabee and Matkin (2012) combined the two approaches by applying didactic instruction, learning activities, group projects, and supervised patient care at a free clinic for the homeless. Similarly, Altshuler et al. (2003) applied a workshop and a culture OSCE. Most researchers used reflective learning methods such as journal-writing and analysis of experiences, those using a mixed method design retrieved the qualitative data from those writings. Some researchers did not apply 
any interventions as the study design was descriptive (Hamre, 2012, Kruse et al., 2014, Larson, 2011).

The heterogeneity of the interventions has been described as well by researchers referring to other theoretical foundations and using various measurement instruments. Educators have developed programs and educational curricula to increase the nurses' and nursing students' intercultural competence. These programs take a variety of approaches, from service-learning (e.g., Cupelli, 2016) and international health promotion experience (e.g., Harrowing, Gregory, O’Sullivan, Lee, \& Doolittle, 2012) to study abroad (e.g., Rolls, Inglis, \& Kristy, 2014; Taylor, Papadopoulos, Dudau, Maerten, Peltegova, \& Ziegler, 2011) to educational programs housed within nursing education programs (e.g., Brown et al., 2008). Hawala-Druy and Hill (2012) aimed to design and implement creative, evidence-based interdisciplinary educational activities that promote positive and culturally competent learning outcomes for millennial students and created an experimental course to investigate ways of addressing the elements they found lacking in current health care education. The results of IAPCC-SV by Campinha-Bacote (2018c) showed that because of course participation and inter-professional educational activities, the level of cultural competency among health profession students improved posteducational intervention. Collectively, the five constructs of cultural knowledge, attitudes, desire, encounter, and skills were significantly progressed by students who completed the course. According to students' feedback, engaging in an interdisciplinary course that utilized eclectic and culturally congruent teaching-learning strategies that 
addressed and matched diverse millennial and learner needs, as well as enthusiastic culturally competent teachers, provided both breadth and depth of learning.

Lonneman (2015) conducted a quantitative study by including a quasiexperimental design using a nonprobability convenience sample of 40 second-degree nursing students at a Midwestern, Catholic university in the United States. Three previous cohorts of students in the same program served as the control groups. The author taught the intervention group and control group 2, whereas another faculty member taught control groups 1 and 3; a similar curriculum was used in all three cohorts. The intervention in this study used six teaching-learning strategies grounded in critical pedagogy and/or transformative learning to increase bi-directional cultural awareness. The strategies involved all students in a variety of activities including direct participation, observation of others' experiences, interviews with patients, group discussion, and private reflection. To evaluate the interventions, the researchers used the third section of the Transcultural Self Efficacy Tool (Jeffreys, 2000; Jeffreys \& Dogan, 2010) and a researcher-designed survey, which asked the students to rate their experience of each of the six teaching strategies for its effectiveness in raising their cultural awareness. Lonneman (2015) reported that the teaching strategies used in this intervention seemed to be effective in increasing students' cultural awareness and are also useful in improving other essential nursing skills, such as communication, ethical reflection, and critical thinking.

Repo, Salminen, and Leino-Kilpi (2017) conducted a study with a correlational descriptive design to evaluate the basic level of cultural competence of graduating 
nursing students in Finland and to identify associated background factors to cultural competence, and furthermore to establish whether teaching multicultural nursing was implemented in nursing education. Their study was based on the Papadopoulos, Tilki, and Taylor Model of Developing Cultural Competence consisting of four stages: cultural awareness, cultural knowledge, cultural sensitivity, and cultural competence (Papadopoulos, 2008). The authors assessed all 295 third- or fourth-year nursing students from four nursing schools by using the Cultural Competence Assessment Tool by Papadopoulos, Tilki, and Ayling (2008). The majority of the participants (74\%) were on a culturally aware level, while the rest (26\%) were on a culturally safe level. None of them measured at an incompetent or competent level. Participants who were not born in Finland $(p=.009)$, had other than Finnish as their mother language $(p=.001)$, had been exchange students $(p=.024)$, and interacted with different cultures daily in their leisure time $(p=.002)$ and on duty $(p=.041)$ scored significantly higher on cultural competence in total. Also, the same background variables were examined for each subsection. Similar associations were identified, mostly in the cultural awareness section. Furthermore, receiving education on multicultural nursing was found to be significantly associated with higher scores in cultural awareness $(p=.022)$ and cultural sensitivity $(p=.014)$. Ability to speak at least one foreign language $(p=.01)$ explained cultural competence in total and the cultural sensitivity subsection $(p=.000)$. Mother language other than Finnish explained cultural awareness $(p=.004)$ and cultural knowledge $(p=.022)$. Exchange studies during nursing education $(p=.048)$ also explained cultural awareness. The frequency of interacting with different cultures in leisure time $(p=.001)$ explained 
the cultural practice. Repo et al. (2017) concluded that "rather than teaching specific knowledge and skills related to different cultures, the emphasis of nursing education should be on professional ethics and moral obligations of encountering all patients in an equal and respectful manner as well as promoting human rights and reducing health inequalities in a global perspective" (p. 104).

The most recent study conducted by Baghdadi and Ismaile (2018) investigated within 461 nursing faculty across the United States. They used the Cultural Diversity Questionnaire for Nurse Educators Revised (Baghdadi \& Ismaile, 2018). The Overall Cultural Competence mean $=166$ indicated that nursing faculty has a moderate level of cultural competence. The highest index was Cultural Knowledge Subscale $M=43.53$, and the lowest index was Cultural Encounter Subscale $M=22.50$. The study identified previous cultural exposure and cultural education as crucial factors to improve cultural competence. However, Kardong-Edgren et al. (2010) compared diverse curriculum approaches within health care education. According to their finding, they concluded that "no one curricular approach appears to be superior for teaching cultural content. Students scored in the culturally aware range, regardless of the approach or educational strategies used by individual programs" (p. 284)

The duration of the studies. Because the time between the pre- and the posttest was different according to the various duration of the interventions the duration of the studies was heterogeneous. Halm et al. (2012), Halm and Wilgus (2013), Harder (2018), and Peying et. al (2012) assessed the participants' orientation toward difference and commonality twice within a short time period of some weeks while Huckabee and 
Matkin (2012) investigated over a five-year-period and Boggis (2012) over a three-year period. Although Munoz et al. (2009) also applied an intervention, they assessed the intercultural competence once more or less at the start of the intervention.

The design of the studies. Altshuler et al. (2003), Boggis (2012), Halm et al. (2012) Harder (2018), and Kruse et al. (2014) used a quantitative design. Bourjolly et al. (2015), Halm and Wilgus (2013), Hamre (2012), Huckabee and Matkin (2012), Larson (2011), and Peying et al. (2012) used a mixed method design. Munoz et al. (2009) used a qualitative design. Most researchers conducted a cross-sectional study including a preposttest design and applied a descriptive and correlational statistical analysis.

The content of the studies. Intentionally, all the studies used IDC by Hammer (2012) based on the DMIS by Bennett (2011) as a theoretical framework and applied the IDI accordingly because only these studies have been selected. There are many other empirical studies reporting results of the intention to foster the intercultural competence within health care providers using all kind of theoretical frameworks and measurement instruments. However, the researchers also implemented other theories within their interventions such as Altshuler et al. (2003) using Hofstede (1980) or Munoz et al. (2009) using Campinha-Bacote's (2002) theory.

The scope of the studies. The researchers investigated within various health care professionals. Altshuler et al. (2003) investigated within pediatrics physicians, Boggis (2012) within students of occupational therapy and a control group of 25 other health professional students, Bourjolly et al. (2015) within mental health providers, Halm and Wilgus (2013) within nursing staff, Halm et al. (2012) within interprofessional team 
members, Hamre (2012) and Harder (2018) within nursing students, Huckabee and Matkin (2012) within physician assistant students, Kruse et al. (2014) within nursing students, faculty, and staff, Larson (2011) within nursing faculty, Munoz et al. (2009) within students of nursing, education and social-work, Peying et al. (2012) within students of physiotherapy, occupational therapy. All studies were conducted in the United States except the study by Peying et al. (2012) that was conducted in Australia and the study by Harder (2018) that was conducted in Canada. No study using the IDI conducted in other parts of the world such as Europe has ever been published in English or German within the scope of health care.

\section{The findings of the studies about the orientation toward cultural difference}

and commonality. Regardless of the intervention, the duration or the scope of the study, the findings of all the studies using the IDI demonstrate minimization as the major developmental orientation toward difference and commonality within the participating health care professionals (Altshuler et al., 2003; Boggis, 2012; Bourjolly et al., 2015; Halm and Wilgus, 2013; Halm et al., 2012; Hamre, 2012; Harder, 2018; Huckabee and Matkin, 2012; Kruse et al., 2014; Larson, 2011; Munoz et al., 2009; and Peying et al., 2012). This transition from ethnocentricism to ethnorelativism typically includes a phase of minimizing cultural differences, where individuals focus more on the similarities between people groups (Bennett, 1993, Hammer, 2012). This minimization of cultural differences may avoid cultural clashes but creates a barrier to exploring unique distinctions between cultures. Opportunities to distinguish unique attributes of different cultures as well as developing frameworks for understanding their own individual 
cultures may help offset the tendency toward minimization (Bennett, 1993). According to Huckabee and Matkin (2012), students should develop their own individual cultural selfawareness to avoid minimization. The authors of the selected studies agree that moving professionals forward on the continuum of increased cultural competency remains the goal, going beyond the zone of minimization. The researchers recommended that further studies should explore the antecedents that contribute to the desire to become more culturally competent as a vital construct that forms the genuine pursuit of the awareness, knowledge, and skills needed (Huckabee and Matkin, 2012).

The findings of the studies about participants' demographic variables. The findings of the selected studies using the IDI will be reported. Also, the findings of studies investigating within health care providers using other theoretical frameworks and measurement instruments in the last ten years will be summarized in order to highlight previous research.

Variables gender and age. Altshuler et al. (2003) was the only author presenting findings regarding the gender of the participants in relation with the results of the IDI and no study presented results about the participants' age. Altshuler et al. (2003) found that gender and multicultural experiences can influence one's level of intercultural sensitivity as the results showed that the female residents exhibited lower Denial and Defense and higher acceptance, cognitive adaptation, and behavioral adaptation compared to their pre-training scores. Males, however, showed an increase in Denial and Defense and a decrease in acceptance, cognitive adaptation, and behavioral adaptation. 
Riley, Smyer, and York (2012) investigated within 53 students enrolled in the first nursing course in a collaborative, online $\mathrm{BSN}$-completion program during three contiguous semesters using the Inventory for Assessing the Process of Cultural Competency Among Healthcare Professionals-Revised (IAPCC-R) by Campinha-Bacote (2018b). This was the first study to investigate and describe the self-reported cultural competence of RN-BSN nursing students alone. IAPCC-R scores ranged from 52 to 91 points $(M=75.3, S D=7.59) ; 26$ participants were recognized as culturally aware, and 26 were recognized as culturally competent. The total mean score is slightly higher than in most previous studies using the IAPCC-R, but barely crossed into the level of cultural competency, which begins at 75 points as defined by Campinha-Bacote (2002). Of Campinha-Bacote's (2002) five constructs, the highest mean scores occurred in the cultural desire construct, with the lowest scores in the cultural knowledge construct. Several correlations were also found between individual constructs. No significant relationships were revealed between gender and total scores or any of the construct scores $(p=.05)$. ANOVA tests identified a significant difference in cultural skill construct scores, with the youngest respondents, aged 20-30 years, recording the highest mean scores. No other significant differences were discovered about age.

De Beer and Chipps (2014) investigated 105 registered nurses working in eight critical care units in a public hospital in KwaZulu-Natal Province. The overall cultural competence mean score for the respondents was $70.2(S D=7.2)$ out of a possible 100 , with $77(74 \%)$ of the respondents scoring in the awareness range, $26(25 \%)$ in the competent range, and only one in the proficient range. No significant differences for 
cultural competence were found for age, gender, qualification, marital status, intensive care unit type or rurality at birth.

Diaz, Clarke, and Gatua (2015) assessed the cultural competence of nurse educators and nursing education programs throughout the state of Wyoming. One hundred and two participants (26 master's level nursing students, 35 community college nursing faculty, 24 university nursing faculty, and 17 clinical educators) filled out the IAPCC-R by Campinha-Bacote (2018b). Multiple linear regression was calculated to predict participant level of cultural competence based on age, highest nursing degree, some years in the nursing field, and levels of nursing degrees. None of the variables tested could be used to predict cultural competence levels of nurse educators, clinical educators, or graduate students in the study sample. Riley et al. (2012) found significant differences between total score and age, as well as a higher total, mean score (75.3) compared with what Diaz et al. (2015) reported in their study (71.6). However, the sample from Riley et al. (2012) of RN/BSN students was younger and practiced in an urban area, in which there was potential for students.

Most recently, Baghdadi and Ismaile (2018) investigated within 461 nursing faculty across the United States and demonstrated by using regression analysis that demographic variables were predictive of the mean score. Similarly, Cruz et al. (2018) conducted a study to (a) assess the cultural competence of nursing students from nine countries; (b) examine the association between the respondents' characteristics and cultural-related background with their cultural competence; and (c) identify the influence of the respondents' characteristics and cultural-related background on the cultural 
competence score. They investigated within 2,163 Bachelor of Science in nursing (BSN) students $($ response rate $=87.3 \%)$ students from nine countries: Chile, India, Iraq, Oman, Philippines, Saudi Arabia, South Africa, Sudan, and Turkey using the Cultural Capacity Scale (Perng \& Watson, 2012). The findings demonstrated a moderate range of cultural competence among the students. Country of residence, gender, age, year of study, attendance at cultural-related training were among others identified as factors affecting cultural competence.

\section{Variable previous education as a health care assistant (having nursing}

experience). None of the selected studies using the IDI within health care providers presented any findings of the relationship between the health care providers using other theoretical frameworks and measurement instruments in the last ten years will be summarized in order to highlight the identified previous research.

Based on Leininger's (1971) model Cicolini et al. (2015) investigated in Italy within 1,432 nurses who completed the Cultural Competence Assessment tool (CCA) by Doorenbos, Schim, Benkert, and Borse (2005). Over 70\% were female; $42.6 \%$ ranged in age from 41 to 50 years, and 50.0\% were bachelor's prepared. More than $50 \%$ had participated in some kind of cultural diversity training. Overall, cultural competence was moderate, showing a moderately high level of cultural awareness and sensitivity $(M=$ $5.41, S D=0.66)$ and a moderate level of culturally competent behaviors $(M=4.33, S D=$ 1.10). Analysis showed that educational level and years of clinical experience were significantly associated with CAS and CCB. In particular, the mean scores increased with level of education ( $p<.001$ for both scores) and with years of experience $(p=.01$ for 
CAS, and $p=.02$ for CCB). Moreover, data showed a significant association between CCB score and prior diversity training $(p<.001)$. Participation in more than one training event was also associated with higher CCB scores $(p<.001)$. Considering the geographical areas, nurses form northern Italy showed higher CAS scores compared with those from the center and south-islands $(p<.001)$.

Riley et al. (2012) investigated within 53 students enrolled in the first nursing course in a collaborative, online $\mathrm{BSN}$-completion program during three contiguous semesters using the IAPCC-R by Campinha-Bacote (2018b). Individual construct scores were also examined for correlation to years of experience; moderate, negative correlations were found between years of experience and both the cultural knowledge construct $(r=-.34 ; p=.05)$ and the cultural skill construct $(r=-.35 ; p=.05)$. A weak, negative correlation existed between years of nursing experience and total IAPCC-K scores $(r=-.29, p=.05)$.

Alpers and Hanssen (2014) investigated at a hospital in Norway with a 30-40\% ethnic minority patient population by using an exploratory mixed method design. The nurses working in a medical unit and psychiatric unit were included in the study independent on age and whether having completed any post-bachelor education. The authors found out that there were no significant differences between the two groups and neither the years of work experience, not the respondents' ages did affect the responses. Corresponding with the findings, the authors concluded that "experience alone does not equip nurses with adequate knowledge for intercultural symptom assessment and culturally competent treatment and care" (p. 1003). The nurses need to be guided by 
reflection on their particular context to learn from experienced situations to become more interculturally competent.

\section{Variable previous education as a health care assistant (having experienced}

diversity at workplace). Mareno and Hart (2014) compared the level of cultural

awareness, knowledge, skills, and comfort of 365 nurses with undergraduate and graduate degrees when encountering patients from diverse populations in the Southeastern States (USA). The researchers based their study on the Process of Cultural Competence in the Delivery of Healthcare Services model by Campinha-Bacote (2002) and used the IAPCCR by Campinha-Bacote (2018b) to assess the intercultural competence of the participants. Also, comparisons were made regarding the amount of cultural diversity training completed between the two nursing groups. Undergraduate-degree nurses scored lower than graduate-degree nurses on cultural knowledge. Scores on cultural awareness, skills, and comfort with patient encounters did not vary between groups. Both groups of nurses reported little cultural diversity training in the workplace or professional continuing education.

Cruz, Estacio, Bagtang, and Colet (2016) investigated within 332 Bachelor of Science in Nursing (BSN) students registered in three Colleges of Nursing in the northern Philippines by using the Cultural Capacity Scale by Perng and Watson (2012). The respondents manifested appreciably good cultural competence $(M=68.98, S D=11.73)$. The ability to understand the beliefs of various cultural groups received the highest score $(M=3.65, S D=0.86)$, while the ability to identify the care needs of patients with diverse cultural backgrounds received the lowest score $(M=3.31, S D=0.74)$. Living in an 
environment with culturally diverse people, prior diversity training, being in the latter years of the nursing program, and with experience of caring for patients from diverse cultures and special population groups, were identified as predictors, accounting for $68.1 \%$ of the variance of cultural competence. Most recently, Cruz et al. (2018) investigated 2,163 Bachelor of Science in nursing (BSN) students from nine countries using the Cultural Capacity Scale (Perng \& Watson, 2012). The researchers identified the experience of taking care of patients from culturally diverse backgrounds and patients belonging to special population groups and living in a multicultural environment were identified as factors affecting cultural competence.

Chan and Nyback (2015) conducted a qualitative study to explore and describe how a virtual course in intercultural communication between 10 Junior year nursing students of Hongkong in China and ten students of Finland, through internationalization at home, can support the development of cultural competence. The collected data consisted of the 20 students' assignment, the joint assignment, and the individual reflections submitted after the seminar. The authors identified the four major themes (a) virtual caravan, (b) commitment, (c) inclusiveness, and (d) perception with understanding. Chan and Nyback (2015) concluded that the students learned to appreciate each other as people with cultural differences yet with much in common and to recognize their assumptions and the value of subtleties and nuances through experiential intercultural encounters without leaving home.

Regarding minorities in health care, the American Association of Colleges in Nursing (Sullivan Commission, 2017) reported recently: "Nursing's leaders recognize a 
strong connection between a culturally diverse nursing workforce and the ability to provide quality, culturally competent patient care. Though nursing has made great strides in recruiting and graduating nurses that mirror the patient population, more must be done before adequate representation becomes a reality. The need to attract students from underrepresented groups in nursing - specifically men and individuals from African American, Hispanic, Asian, American Indian, and Alaskan native backgrounds - is a high priority for the nursing profession." (Enhancing Diversity in the Workforce, para 1).

Variables country of citizenship, ethnic minority group affiliation, migration background, and total amount of time having lived in another country. Riley et al. (2012) investigated 53 students enrolled in the first nursing course in a collaborative, online BSN-completion program during three contiguous semesters using IAPCC-R by Campinha-Bacote (2018b). Statistical analysis did not identify any significant relationships between race and IAPCC-R scores. De Beer and Chipps (2014) investigated 105 registered nurses working in eight critical care units in a public hospital in KwaZuluNatal Province. Nurses from non-English-speaking backgrounds scored significantly higher in cultural competence than English-speaking nurses $(M=66.3, S D=6.0$ versus $M=71.8, S D=7.1)$. Analyzing the data by English-speaking background, the higher cultural competence score for non-English-speaking nurses (primarily isiZulu-speaking) was driven by significant differences in cultural encounters $(p<.001)$, cultural desire $(p=.02)$ and cultural awareness $(p=.02)$. Furthermore, significant differences were found for the race $(K=20, p<.001)$, with black nurses scoring significantly higher $(M=$ 72.2, $S D=6.9)$ compared with the other races $(M=64.1-66.0)$. 


\section{Summary and Conclusions}

Diversity is inherent in the Swiss health care system. Nurses with an orientation toward cultural difference and commonality in an ethnorelative stage on the IDC are able to deal more effectively with cultural diversity as those nurses with an orientation toward cultural difference and commonality in an ethnocentric stage (Hammer, 2012). Therefore, the nursing students' development of intercultural competence needs to be understood. Previous studies report that a majority of the participating health care providers have an orientation toward cultural difference and commonality in minimization representing a transition stage between the ethnocentric and the ethnorelative worldview. Knowledge of what fosters the movement of the nursing students along the IDC is limited due to heterogeneity regarding content, intervention, duration, scope in previous studies.

Therefore, there is still a need to know more about the contributors to the development of intercultural competence, particularly within nursing.

Applying a secondary data analysis this quantitative research study is an investigation into the effect of a specialized program integrated into the curriculum at a Nursing College on the nursing student-cohorts' orientation toward cultural difference and commonality. Furthermore, the relationship between the individual nursing students' orientation toward cultural difference and commonality and the demographic factors of the nursing students such as age, gender, country of citizenship, ethnic minority group affiliation, migration background, world region having primarily lived up to age 18 , total amount of time having lived in another country, having had lessons about own culture or any foreign cultures, and previous education as a health care assistant will be examined. 
This study will affect positive social change by providing more knowledge to understand the contributors to an increase in intercultural competence. Consequently, nursing educators may use this knowledge to better understand the contributors to the development of intercultural competence of nursing students. 
Chapter 3: Research Method

\section{Introduction}

The purpose of this quantitative cross-sectional study was to identify the orientation toward cultural difference and commonality on the IDC of nursing students at a nursing college in Switzerland, providing more knowledge about contributors to intercultural competence. The nursing students at the nursing college in Switzerland completed the IDI (2018a, 2018b) between 2010 and 2016 at the start-point and the endpoint of their 2, 2.5, 3, or 4-year education. This study was conducted to examine the effect of a specialized program at a nursing college on the nursing students' orientation toward cultural difference and commonality and the relationships to the nursing studentcohorts' demographic variables of duration of the education, year of curriculum, workload, focus of specialization, and location of school lessons. Furthermore, the relationship was also examined between the nursing students' orientation toward cultural difference and commonality and the demographic factors of age, gender, country of citizenship, ethnic minority group affiliation, migration background, world region lived in up to age 18, amount of time living in another country, lessons about culture, and previous education as a health care assistant.

This chapter will provide information about the research design and rationale, the population, and the sample included in this study. Furthermore, the instrumentation and operationalization of the constructs will be explained in detail. The threats to validity will be considered before the ethical procedures will be highlighted. A summary will conclude this chapter. 


\section{Research Design and Rationale}

This study was a quantitative investigation of the contributors to intercultural competence of nursing students. Because the data were collected between 2010 and 2016, a secondary data analysis was conducted. Accordingly, this study includes an ex post facto design by using data already collected but not collected for research purposes. The ex post facto design is appropriate when it is not possible to manipulate the characteristics of human participants (Simon \& Goes, 2013). This was the case in this study, as the independent variables regarding the nursing students who provided their data between 2010 and 2016 could not be manipulated.

The ex post facto design has similar logic of inquiry to experimental research designs, meaning it is a substitute for experimental research and can be used to test hypotheses about cause-and-effect or correlational relationships (Simon \& Goes, 2013). Secondary data analysis was used to explain the effect on the nursing students' orientation toward cultural difference and commonality and its relationship to their demographic variables based on an condition that every nursing student completed the specialized program integrated into the curriculum at a nursing college in Switzerland. Analysis also included the relationship between nursing students' orientation toward cultural difference and commonality and the demographic factors of the nursing students. The influence on variables were determined with the examination of occurring correlations by using statistical hypothesis testing techniques. This investigation was essential, as educators need knowledge about how individuals, teams, and organizations 
can increase intercultural competence to achieve educational and organizational goals (Hammer, 2015b).

\section{Methodology}

\section{Population}

The population was nursing students at the largest college of higher education in nursing in Switzerland. Every year, the staff of more than 250 dedicated professionals train around 400 students to become registered nurses. The development of the intercultural competence has been integrated into the curriculum since 2010. Trainers perform training sessions with the nursing students during their entire education. In the program, nursing students complete a student exchange program in the last year of their higher education to the nursing diploma. An accurate preparation for the exchange and reflection during and after the exchange is emphasized to increase students' intercultural competence (Vande Berg, 2007,2009; Vande Berg, Balkcum, Scheid, \& Whalen, 2004; Vande Berg, Connor-Linton, \& Paige, 2009). The nursing college uses the IDI (Hammer, 2018 b) to measure the developmental orientation score of the nursing students at the start and the end of their education.

There is a regular education leading to a diploma in nursing that lasts 3 years full time or 4 years part time. If students previously completed education as a health care assistant, they are allowed to shorten their education (OdASanté, 2018). Between 2010 and 2012, the shortened education lasted 2 and a half years and between 2013 and 20162 years full time and 4 years part time. Between September 2010 and September 2016, the number of students who started their nursing education at the nursing college was 2,373. 
Out of these students, 1,323 students completed their education within this time frame. The difference between these numbers is because 108 students dropped out, and 1,050 students who started their education within this study's time frame completed their education later than September 2016 (adjustments were made after obtaining the data).

\section{Sample}

Nursing students have completed the IDI at the start-point and the end-point of their education lasting either 2, 2.5, 3, or 4 years. The data for the demographic variables were provided by the nursing students in response to additional questions within the IDI. The following data were available for the secondary data analysis (adjustments have been made after obtaining the actual data):

- 2,298 students who completed the IDI at the start point of education

- 1,112 (out of 2,298) students who completed the IDI at the end-point of education

- 1691 students (out of 2,298) who completed a regular 3-year education

- 719 students (out of 2,298) who completed a shortened 2.5-year education

- 933 students (out of 2298) who completed a shortened 2-year education

- 67 students (out of 2298) who completed a part-time 4-year education

\section{Procedure for Gaining Access to the Data Set}

Several meetings were arranged with the responsible persons at the nursing college. With the first meeting, the executive director wanted the involvement of the two persons below him on the hierarchy. Accordingly, there was another meeting with the head of the department of international relations and diversity and another meeting with 
the head of the department of preregistration education. All of them were generally interested but wanted to consider the legal regulations in Switzerland. In this regard, the law in Switzerland allowed for the secondary data analysis without explicit informed consent from the data provider as long as the data is deidentified, which is what the IRB at Walden University required as well. There was another meeting with the head of the department of international relations and diversity and her deputy to discuss the research questions, hypotheses, and methods. As the head of the department international relations and diversity served as the qualified administrator to use the IDI, she ensured that the executive director was allowed to sign the data agreement by asking Dr. Mitchell Hammer. Shortly before, Dr. Mitchell Hammer approved the use of the IDI for this research study according to the detailed proposal (see Appendix A).

After all these meetings and clarifications, the decision was made by all the involved responsible to give permission to use all data collected from the nursing students between 2010 and 2016. The data that have been collected since March 2017 at the nursing college were excluded, because they have not been collected anonymously anymore, and the deidentification of this large amount of data would have been too time intensive. The executive director of the nursing college gave permission to use the data for a secondary data analysis and signed an agreement for operational oversight and data use.

To meet the requirement of IDI, the company hosting the data, a reassignment of the anonymous data included in this study from the personal account (of the head of the department internationalization and diversity at the college) to an enterprise account was 
necessary. A further requirement was to participate at a 2-day-seminar to become a qualified administrator of the IDI that was completed in November 2017.

\section{Instrumentation and Operationalization of Constructs}

Assessing intercultural competence is similarly complex, and multiple pathways are possible. Hammer (2015a) argued that "most approaches have been compositional, identifying or listing personal characteristic (e.g., trait) components of intercultural competence, with the little theoretical specification of how the identified components relate to one another." (p. 484). Accordingly, assessment attempts to measure to what degree individuals possess these traits, which often fall under the widely-used classification of cognitive, affective, and behavioral.

The IDI was selected for this study due to its demonstrated theoretical grounding on the DMIS originally proposed by Bennett (1986, 1993, 2004, 2011). This instrument allows users to assess the effectiveness of various cross-cultural intervention by measuring the respondents' change within the development of their intercultural competence. A second consideration for utilizing the IDI was that numerous reports have documented that this assessment is cross-culturally generalizable, valid and reliable (Hammer, 2008/2010/2015c; Hammer et al., 2003; Paige, Jacobs-Cassuto, Yershova, \& DeJaeghere, 2003; Wiley, 2017). Content validity of the original 60-item IDI was established through in-depth interviews with individuals from a variety of cultures. Evaluations by a panel of experts, followed by survey pilot testing, reduced the original scale from 60 to 50 items that cross five factors: denial/ defense (DD), reversal (R), 
minimization (M), acceptance/adaptation (AA), and encapsulated marginality (EM). Reliability coefficients or all five scales ranged from 0.80-.085 (Hammer et al., 2003).

\section{Threats to Validity}

The students completed the IDI within a regular school lesson. At the start-point of their education, they might not have understood very well the term culture although it is explained within the IDI. At the end-point of their education, the concentration to complete the IDI may have been threatened because the students came to say goodbye to each other for this very last day to school after having completed the last 6-monthinternship. As well at the start- as at the end-point, the students were aware that the intention of applying the IDI was to know how their orientation toward cultural difference and commonality is. The Hawthorn Effect may be considered as a threat to validity, as the students may have altered their responses according to what they think we would consider as a good response. The students may have changed their responses being evaluated only because it is being studied, and not because of the intervention (McCambridge, Kyprib, \& Elbourne, 2014). This may have happened although the study has not been designed as an experimental study, the students were aware that the purpose of taking the IDI was to evaluate the specialized program to foster their intercultural competence.

Furthermore, as threat to the validity needed to be considered that there might be other variables confounding the findings of this study. There was no random assignment as every nursing students at the Nursing College between 2010 and 2016 who was present in the lesson where the IDI has been conducted was included in the study. 
Consequently, there could be inherent confounds in the variables studied because of no random assignment, but also the fact that the development of the intercultural competence is dependent on other variables occurring in the students' personal characteristics or their environment as those being included in this study as independent variables.

\section{Ethical Procedures}

For the conduction of the secondary data analysis, the de-identified data of nursing students was used. Two thousand and two hundred and ninety-eight nursing students completed the IDI at the start-point and 1,112 nursing students at the end-point of their two-, two and a half-, three-, or four-year education at the particular Nursing College in Switzerland. All nursing students provided their data anonymously. They were randomly assigned to a login that does not relate to the person. Therefore, the data represent a de-identified dataset. This procedure was consistent with the Swiss regulations regarding data protection. The Federal Data Protection and Information Commissioner (FDPIC) was the competent authority in Switzerland for data processing by federal bodies and private persons, including enterprises. As far as data processing by cantonal or communal authorities is concerned, the supervision is up to the cantonal and communal data protection commissioners (Swiss Confederation, 2018f). For the particular Nursing College, the Data Protection Act of the Cantone of Bern (CDPA) (The Cantonal Parliament of the Canton of Bern, 2006) based on the Swiss Confederation (2018g) is relevant. The Art. 15 regulated the processing of data for research: 
1. A responsible authority may process personal data for purposes not related to specific persons, and in particular for research, practical training, statistics and planning [Version of 31. 3. 2009] if

a. the data is rendered anonymous as soon as the purpose of the processing permits or is at least used without persons being directly identified, and b. the results are published in such a manner that the data subjects may not be identified.

2. The responsible authority may disclose personal data for processing for purposes not related to specific persons if it guarantees that the recipient

a. meets the requirements listed in paragraph 1 ,

b. will not pass the personal data on to third parties and

c. will ensure that the data is secured.

(The Cantonal Parliament of the Canton of Bern, 2006)

The Institutional Review Board (IRB) of Walden University approved to conduct this dissertation research (secondary data analysis) on November 7, 2018 (approval no. 11-0718-0662781).

\section{Summary}

The purpose of this quantitative descriptive cross-sectional study was to identify the orientation toward cultural difference and commonality on the IDC of nursing students in Switzerland and to provide more knowledge about the contributors to the development of intercultural competence. Accordingly, the effect of a specialized program integrated into the curriculum at a Nursing College on the nursing student- 
cohorts' orientation toward cultural difference and commonality will be examined. Furthermore, the relationship between the individual nursing students' orientation toward cultural difference and commonality and the demographic factors of the nursing students such as age, gender, country of citizenship, ethnic minority group affiliation, migration background, world region having primarily lived up to age 18 , total amount of time having lived in another country, having had lessons about own culture or any foreign cultures, and previous education as a health care assistant was examined. The nursing students completed the IDI between 2010 and 2016 at the start and the end of their two-, two and a half-, three, or four-year education. The data for these demographic variables were provided by the nursing students in response to additional questions within the IDI. The research questions led the secondary data analysis of the nursing students' data. 
Chapter 4: Results

\section{Introduction}

The purpose of this quantitative and descriptive cross-sectional study was to identify the orientation toward cultural difference and commonality on the IDC of nursing students in Switzerland and to provide more knowledge about the contributors to intercultural competence. At the college in Switzerland, the development of the intercultural competence is integrated into the curriculum during the entire education. Every nursing student must complete a student exchange program in the last year of their higher education to the nursing diploma, with an emphasis on preparation and reflection to increase their intercultural competence. Training sessions are also integrated into the curriculum throughout the education at the college. With the intention to measure the students' orientation toward cultural difference and commonalities, the IDI has been applied for every student at the college at the start and the end of their education. The following questions led the secondary data analysis of the nursing students' data:

RQ 1: What are the nursing students' orientation toward cultural difference and commonality on the Intercultural Development Continuum?

RQ 2: What is the relationship among the nursing students' demographic variables and the nursing students' orientation toward cultural difference and commonality?

$H_{0}$ : There is no relationship among the nursing students' demographic variables and their stage on the Intercultural Development Continuum. 
$H_{1}$ : There is a relationship among the nursing students' demographic variables and their stage on the Intercultural Development Continuum.

RQ 3: To what extent does the nursing student-cohorts' orientation toward cultural difference and commonality as a group differ from the beginning to the end of their education?

RQ 4: What is the relationship between the difference regarding the nursing student-cohorts' orientation toward cultural difference and commonality at the start-point and end-pont of education and the demographic variables of the cohort (years of education, year of curriculum, workload, focus of specialization, and location of school)?

$H_{0}$ : There are no differences between the orientation toward cultural difference and commonality at the start of the nursing education and the orientation toward cultural difference and commonality at the end-point of the nursing education for the nursing student-cohorts according to the various demographics regarding duration of the education $(2,2.5,3$, or 4 years), year of curriculum (2010 or 2015), workload (full-time or short-time), focus of specialization (physically-ill patients, psychiatry, pediatrics/obstetrics/gynecology), location of school lessons (head location in a larger city or branch location in a smaller city in a mountainous area).

$H_{1}$ : There is a difference between the orientation toward cultural difference and commonality at the start of the nursing education and the orientation toward cultural difference and commonality at the end-point of the nursing education for the nursing student-cohorts according to the various demographics regarding duration of the education (2, 2.5, 3 or 4 years), year of curriculum (2010 or 2015), workload (full-time or 
short-time), focus of specialization (physically ill patients, psychiatry, pediatrics/obstetrics/gynecology), location of school lessons (head location in a larger city or branch location in a smaller city in a mountainous area).

The baseline descriptive and demographic characteristics of the sample and the results of the statistical analysis of the data to test the hypotheses and to provide answers to the four research questions will be presented in this chapter.

\section{Data Collection}

The nursing students at the college completed the IDI between September 2010 and September 2016 at the start and the end of their 2, 2.5, or 3-year education. The results of the IDI (2018a, 2018b) provided the orientation toward cultural difference and commonality as the dependent variable for individual nursing students as well as cohorts. The data for the independent variables were provided by the nursing students in response to additional questions in the IDI. These independent variables are the demographic variables of the individual nursing students such as age, gender, country of citizenship, ethnic minority group affiliation, migration background, world region having primarily lived up to age 18 , total amount of time having lived in another country, having had lessons about own culture or any foreign cultures, and previous education as a health care assistant. The data also showed the nursing student-cohorts' demographics such as duration of the education (2, 2.5, 3, or 4 years), curriculum (2010 or 2015), workload (full time or part time), focus of specialization (physically ill patients, psychiatry, pediatrics/obstetrics/ gynecology), and location of school lessons (head location in a 
larger city or branch location in a smaller city in a mountainous area). This is consistent with what was described in Chapter 3.

\section{Baseline Descriptive and Demographic Characteristics of the Sample}

The IDI took place in a regular school lesson, and every nursing student was required to complete the IDI as required in the curriculum. Students who were absent on the day of this lesson were required to take the inventory afterward. However, some of them did not complete the IDI afterward. At the end-point of education, some students were not present at this last school-day, because they had to repeat their final exams and accordingly, they did not take the IDI. Therefore, the response rate was in many cases $100 \%$ but at the start-point of education also as low as $76.7 \%$ or at the end-point $33.3 \%$ (Appendix B).

There were 2,298 out of 2,373 nursing students of 76 cohorts who started their education between September 2010 and September 2016 and completed the IDI at the start-point of their education. These numbers represent an overall response rate of $96.8 \%$. Until September 2016, 40 (out of these 76) cohorts completed their education. One thousand and one hundred and two out of 1,223 nursing students of these 40 cohorts completed the IDI at the end-point of their education. These numbers represent an overall response rate of $96.8 \%$. The difference between the number of students in a cohort at the start-point and the end-point of their education occurred because of the repetition of a semester during education or drop-outs for other reasons. The difference of the number of cohorts is because 37 cohorts completed their education after the time frame for this 
study. Overall, the sample is representative of the population of interest who are the nursing students at this college.

The following table displays the frequencies of the demographic variables of the individual nursing students such as age, gender, country of citizenship, ethnic minority group affiliation, migration background, world region having primarily lived up to age 18 , total amount of time having lived in another country, having had lessons about own culture or any foreign cultures, and previous education as a health care assistant, are presented. 
Table 3

Frequencies for Demographic Variables

\begin{tabular}{|c|c|c|}
\hline & Frequency $(n)$ & Percent $(\%)$ \\
\hline \multicolumn{3}{|l|}{ Age } \\
\hline 17 and under & 6 & 0.2 \\
\hline $18-21$ & 1,675 & 49.1 \\
\hline $22-30$ & 1,407 & 41.3 \\
\hline $31-40$ & 152 & 4.5 \\
\hline $41-50$ & 120 & 3.5 \\
\hline $51-60$ & 31 & 0.9 \\
\hline 61 and over & 15 & 0.4 \\
\hline Missing data & 4 & 0.1 \\
\hline Total & 3,410 & 100 \\
\hline \multicolumn{3}{|l|}{ Gender } \\
\hline Male & 1,221 & 35.8 \\
\hline Female & 2,141 & 62.8 \\
\hline Missing data & 48 & 1.4 \\
\hline Total & 3,410 & 100 \\
\hline \multicolumn{3}{|l|}{$\begin{array}{l}\text { Primary country of } \\
\text { citizenship }\end{array}$} \\
\hline Swiss citizenship & 2,458 & 89.6 \\
\hline Other citizenship & 269 & 9.8 \\
\hline Missing data & 17 & 0.6 \\
\hline Total & 2,744 & 100 \\
\hline \multicolumn{3}{|c|}{$\begin{array}{l}\text { Member of ethnic minority } \\
\text { in country }\end{array}$} \\
\hline Yes & 207 & 6.1 \\
\hline No & 2,585 & 75.8 \\
\hline Missing data & 618 & 18.1 \\
\hline Total & 3,410 & 100.0 \\
\hline \multicolumn{3}{|c|}{ Migration background } \\
\hline Yes & 571 & 16.7 \\
\hline No & 2,757 & 80.9 \\
\hline Missing data & 82 & 2.4 \\
\hline Total & 3,410 & 100 \\
\hline
\end{tabular}




\begin{tabular}{|c|c|c|}
\hline & Frequency $(n)$ & Percent $(\%)$ \\
\hline \multicolumn{3}{|c|}{ Regions lived in until age 18} \\
\hline North America & 9 & 0.3 \\
\hline Central America & 2 & 0.1 \\
\hline South America & 17 & 0.5 \\
\hline Middle East & 12 & 0.4 \\
\hline Africa & 19 & 0.6 \\
\hline Australia & 4 & 0.1 \\
\hline Asia Pacific & 21 & 0.6 \\
\hline Western Europe & 3034 & 89.0 \\
\hline Eastern Europe & 142 & 4.2 \\
\hline Missing data & 150 & 4.4 \\
\hline Total & 3,410 & 100 \\
\hline \multicolumn{3}{|c|}{$\begin{array}{l}\text { Lived in another country for } \\
\text { a certain amount of time }\end{array}$} \\
\hline $\begin{array}{l}\text { never lived in anothe } \\
\text { country }\end{array}$ & 1,508 & 44.2 \\
\hline less than 3 months & 590 & 17.3 \\
\hline 3-6 months & 510 & 15 \\
\hline 7-11 months & 224 & 6.6 \\
\hline $1-2$ years & 235 & 6.9 \\
\hline $3-5$ years & 107 & 3.1 \\
\hline $6-10$ years & 69 & 2 \\
\hline over 10 years & 153 & 4.5 \\
\hline Missing data & 14 & 0.4 \\
\hline Total & 3,410 & 100 \\
\hline \multicolumn{3}{|c|}{$\begin{array}{l}\text { Lessons about personal or } \\
\text { foreign cultures }\end{array}$} \\
\hline No & 1,012 & 29.7 \\
\hline Less than 1 day & 271 & 7.9 \\
\hline Less than 3 days & 565 & 16.6 \\
\hline Less than 1 week & 494 & 14.5 \\
\hline Less than 2 weeks & 266 & 7.8 \\
\hline More than 2 weeks & 725 & 21.3 \\
\hline Missing data & 77 & 2.3 \\
\hline Total & 3,410 & 100 \\
\hline \multicolumn{3}{|c|}{$\begin{array}{l}\text { Previous education as health } \\
\text { care assistant }\end{array}$} \\
\hline Yes & 1,679 & 49.2 \\
\hline No & 1,065 & 31.2 \\
\hline Missing data & 666 & 19.5 \\
\hline Total & 3,410 & 100 \\
\hline
\end{tabular}


The following table shows the frequencies of the nursing student-cohorts' demographics such as duration of the education $(2,2.5,3$, or 4 years $)$, curriculum (2010 or 2015), workload (full-time or short-time), focus of specialization (physically ill patients, psychiatry, pediatrics/obstetrics/gynecology), and location of school lessons (head location in a larger city or branch location in a smaller city in a mountainous area). 
Table 4

Frequencies for Nursing Cohort Data

\begin{tabular}{lll}
\hline & Frequency $(n)$ & Percent $(\%)$ \\
\hline Duration of nursing education & & \\
\hline 3 years & 1,691 & 49.6 \\
2.5 years & 719 & 21.1 \\
2 years & 933 & 27.4 \\
4 years & 67 & 2 \\
\hline Total & 3,410 & 100 \\
\hline Year of introduction to curriculum & & \\
\hline Curriculum 2010 & 2,773 & 81.3 \\
Curriculum 2015 & 637 & 18.7 \\
Total & 3,410 & 100 \\
\hline Level of employment & & \\
full-time & 3,343 & 98 \\
part-time & 67 & 2 \\
\hline Total & 3,410 & 100 \\
\hline Focus of specialization & & \\
physically ill patients & 3,315 & 97.2 \\
psychiatric patients & 42 & 1.2 \\
pediatric/obstetric/gynecologic patients & 53 & 1.6 \\
\hline Total & 3,410 & 100 \\
\hline Location of school & & \\
\hline head location in a larger city & 2,673 & 78.4 \\
branch location in a smaller city in a & 737 & 21.6 \\
mountainous area & 3,410 & \\
Total & & \\
\hline & & \\
\hline
\end{tabular}




\section{Results to Research Question 1}

RQ 1 What are the nursing students' orientation toward cultural difference and commonality on the Intercultural Development Continuum?

Between 2010 and 2016, 2,298 nursing students completed the IDI at the startpoint of their education and 1,112 nursing students at the end-point (a total 3,410 nursing students who completed the IDI). Using SPSS, the following descriptive statistics were found regarding the nursing students' PO and DO toward cultural difference and commonality on the IDC at the start-point and the end-point of the education. The participants' mindsets were identified according to the scores of the PO and the DO as follows (IDI, 2018b): scores 55.00-65.99 = denial; 66.00-69.99 = cusp of polarization; scores 70.00-82.99 = polarization; scores 83.00-84.99 = cusp of minimization; scores 85.00-111.99 = minimization; scores 112.00-114.99 = cusp of acceptance; scores 115.00-126.99 = acceptance, scores 127.00-129.99 = cusp of adaptation; and scores $130.00-145.00=$ adaptation

The mean of the nursing students had a PO in the mindset acceptance $(M=118.5$, $S D=5.0, \min =101.8, \max =136.6$, variance $=25.0$ at the start-point; $M=119.3, S D=$ $5.4, \min =102.3, \max =140.5$, variance $=29.2$ at the end-point of education $)$ and a DO in the mindset minimization $(M=85.5, S D=13.2, \min =38.1, \max =131.7$, variance $=$ 173.0 at the start-point; $M=87.9, S D=14.7, \min =43.1, \max =136.8$, variance $=204.8$ at the end-point of education).The standard deviation is relatively high for the DO, whereas it is lower for the PO. The standard deviation is a measure of variability or the 
distribution of the variable; the higher above zero the standard deviation is, the more variation there is in the distribution (Frankfort-Nachmias \& Leon-Guerrero, 2015). The values for the variance are high for the DO, meaning the variation among the students' DO is high.

The kurtosis provides information about the peakedness of the distribution, "a measure of whether the data are heavy-tailed or light-tailed relative to a normal distribution" (Engineering Statistics Handbook, 2018, para. 3). The kurtosis value (0.1 for the PO, -0.1 for the DO) indicated a light-tailed distribution relative to a normal distribution of the scores among the 3,410 nursing students. The skewness value indicated the symmetry of the distribution (Pallant, 2016). The skewness value $(0.23$ for the PO, 0.3 for the DO) indicated that the scores are rather clustered to the left at the low values.

The combination of the 2,298 nursing students being assessed at the start-point of their education with those 1,112 nursing students being assessed at the end-point of their education provided the following results: $68 \%$ of all 3,410 nursing students had a PO representing the mindset acceptance $(M=118.8, S D=5.1, \min =113.7, \max =123.9$, variance $=26.5)$ while $68 \%$ of them had a DO representing the mindset minimization $(M$ $=86.3, S D=13.7, \min =72.6, \max =100$, variance $=187.9)$. In summary, the distribution of the PO and DO scores among the nursing students at the start-point and the end-point of education can be considered as normal with most scores occurring in the center, tapering out toward the extremes. This result is also supported by the value of kurtosis and skewness. If the distribution is perfectly normal, the Skewness and Kurtosis 
value of 0 would occur. According to Pallant (2016), this is rather an uncommon occurrence in the social sciences. However, the variation is high for the DO and overall the distribution is light-tailed and rather clustered to the left at the low values.

Accordingly, these observations were found in the frequency distribution of the PO and the DO within the 2,298 students at the start-point, the 1,112 students at the end-point, and overall within the 3,410 students at the start- and end-point combined. The following Tables 5-10 and Figures 6-11 visualize these frequency distributions.

Table 5

Perceived Orientation at the Start-Point and End-Point of the Education

\begin{tabular}{lll}
\hline Perceived orientation & Frequency $(n)$ & $\%$ \\
\hline Minimization & 99 & 2.9 \\
Cusp of acceptance & 702 & 20.6 \\
Acceptance & 2,369 & 69.5 \\
Cusp of adaptation & 168 & 4.9 \\
Adaptation & 72 & 2.1 \\
\hline Total & 3,410 & 100 \\
\hline & & \\
\hline & & \\
\hline
\end{tabular}

Figure 6. Distribution of the score of perceived orientation at the start-point and the endpoint of the education. 
Table 6

Developmental Orientation at the Start-Point and End-point of the Education

\begin{tabular}{lll}
\hline Developmental orientation & Frequency $(n)$ & $\%$ \\
\hline Denial & 199 & 5.8 \\
Cusp of polarization & 152 & 4.5 \\
Polarization & 1,043 & 30.6 \\
Cusp of minimization & 329 & 9.6 \\
Minimization & 1,499 & 44 \\
Cusp of acceptance & 101 & 3 \\
Acceptance & 82 & 2.4 \\
Cusp of adaptation & 2 & 0.1 \\
Adaptation & 3 & 0.1 \\
\hline Total & 3,410 & 100 \\
\hline
\end{tabular}

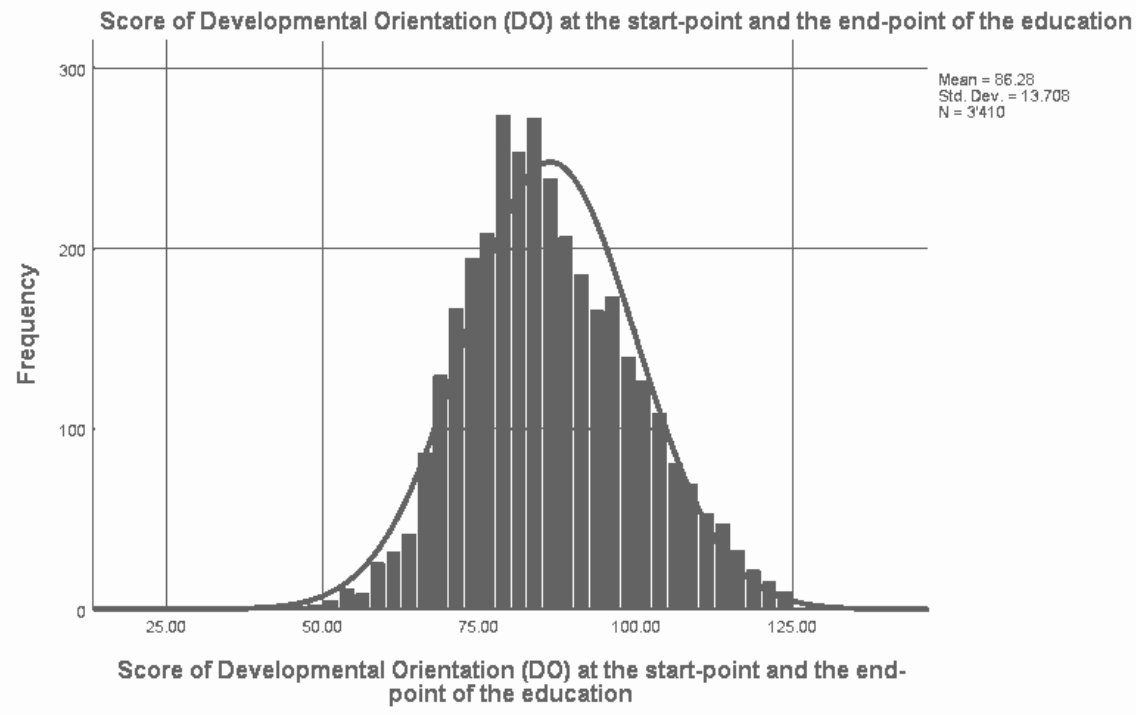

Figure 7. Distribution of the score of developmental orientation at the start-point and the end-point of the education.

The frequency distribution of the PO and DO supported the result regarding skewness and kurtosis. For instance, Table 6 and Figure 7 show the distribution of the 
DO within the 3,410 students with the highest values for the mindsets of minimization (representing the middle) and Polarization (representing a lower value more on the left) (kurtosis $=-0.1)$ indicating a light-tailed distribution being more left from the center. The distribution clustered to the left at the low values ( three of the 3,410 nursing students represented a DO mindset of adaptation on the far right while a considerable number, precisely 199 students, represented a DO mindset of denial on the far left (Table 6). The frequency distribution of the PO and the DO is presented separately for the start-point and the end-point of education in the Tables 7-10 and the Figures 8-11.

\section{Table 7}

Perceived Orientation of the Students at the Start-Point of the Education

\begin{tabular}{lll}
\hline Perceived orientation & Frequency $(n)$ & $\%$ \\
\hline Minimization & 73 & 3.2 \\
Cusp of acceptance & 483 & 21 \\
Acceptance & 1,605 & 69.8 \\
Cusp of adaptation & 104 & 4.5 \\
Adaptation & 33 & 1.4 \\
\hline Total & 2,298 & 100 \\
\hline
\end{tabular}




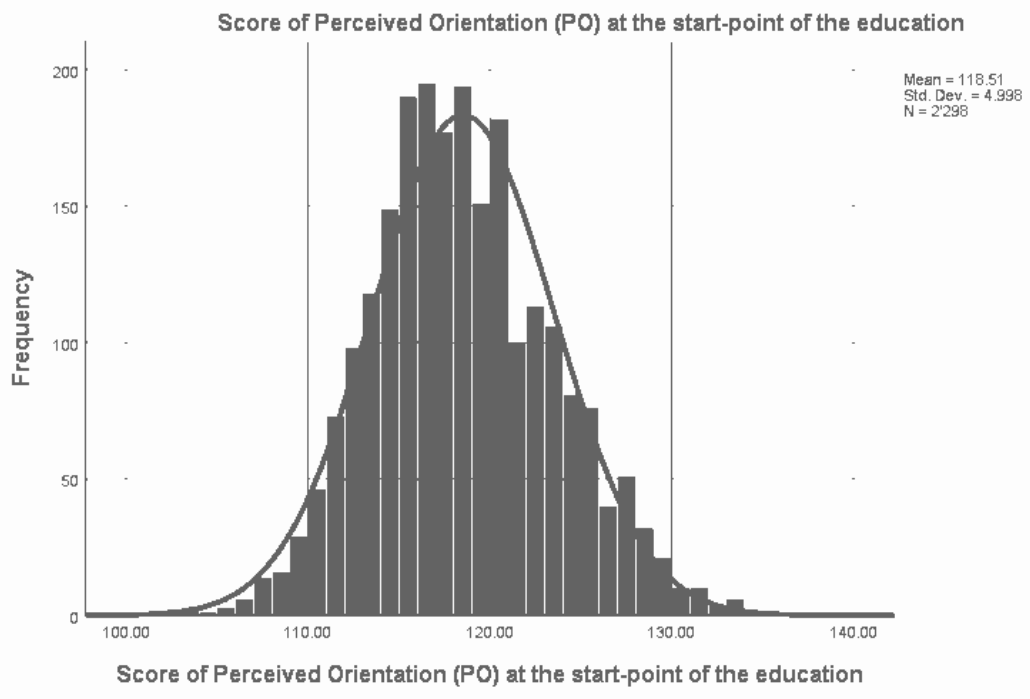

Figure 8. Distribution of the score of perceived orientation at the start-point of the education.

Table 8

Developmental Orientation of the Students at the Start-Point of the Education

\begin{tabular}{lll}
\hline Developmental orientation & Frequency $(n)$ & $\%$ \\
\hline Denial & 144 & 6.3 \\
Cusp of polarization & 102 & 4.4 \\
Polarization & 735 & 32 \\
Cusp of minimization & 230 & 10 \\
Minimization & 1,001 & 42.6 \\
Cusp of acceptance & 47 & 2 \\
Acceptance & 36 & 1.6 \\
Cusp of adaptation & 2 & 0.1 \\
Adaptation & 1 & 0 \\
Total & 2,298 & 100 \\
\hline
\end{tabular}




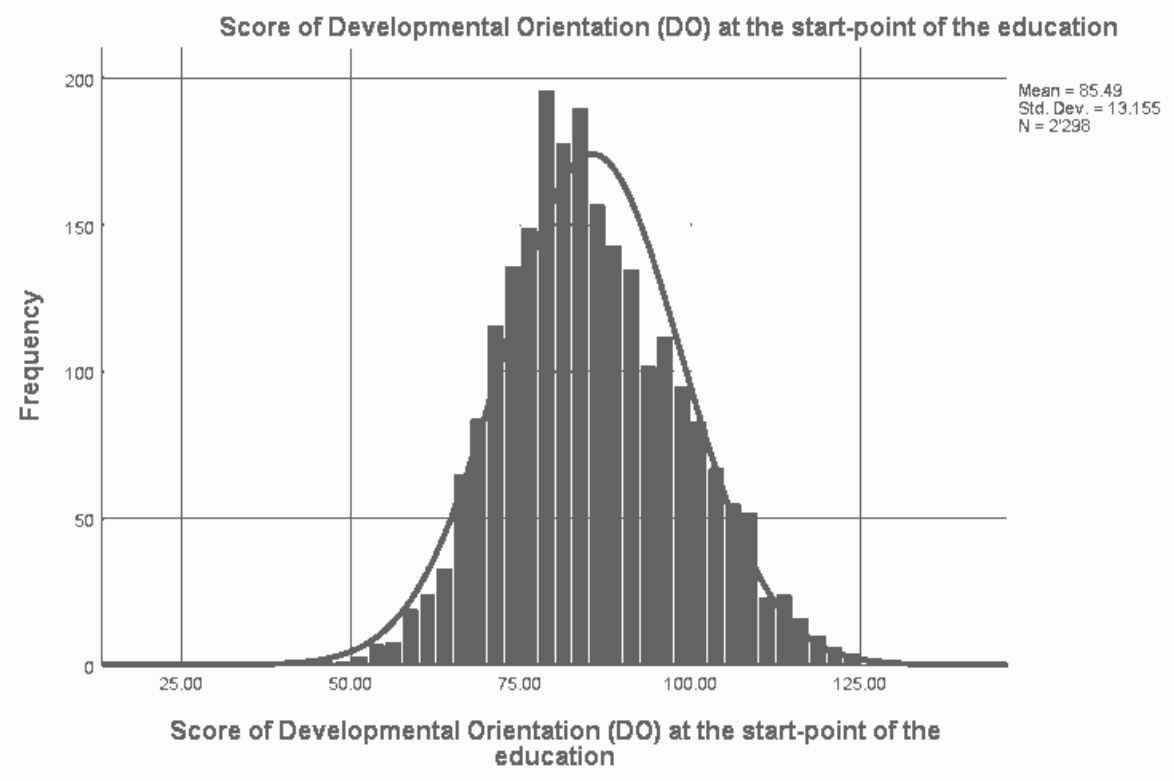

Figure 9. Distribution of the students' score of developmental orientation at the startpoint of the education.

Table 9

Perceived Orientation of the Students at the End-Point of the Education

\begin{tabular}{lll}
\hline Perceived orientation & Frequency $(n)$ & $\%$ \\
\hline Minimization & 26 & 2.3 \\
Cusp of acceptance & 219 & 19.7 \\
Acceptance & 764 & 68.7 \\
Cusp of adaptation & 64 & 5.8 \\
Adaptation & 39 & 3.5 \\
Total & 1,112 & 100 \\
\hline
\end{tabular}




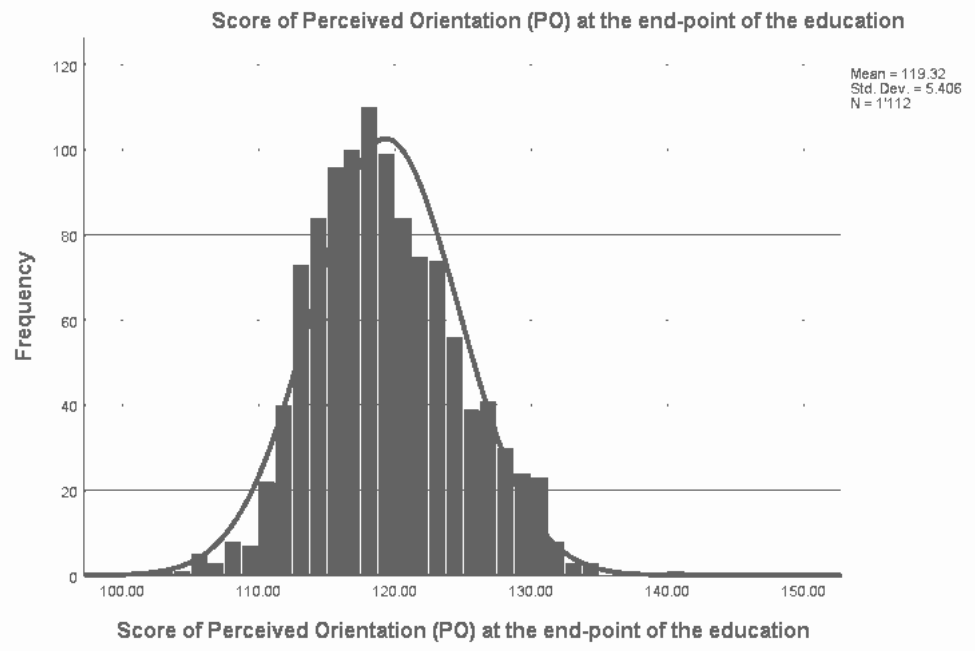

Figure 10. Distribution of the students' score of perceived orientation at the end-point of the education.

Table 10

Developmental Orientation of the Students at the End-Point of the Education

\begin{tabular}{lll}
\hline Developmental orientation & Frequency $(n)$ & $\%$ \\
\hline Denial & 55 & 4.9 \\
Cusp of polarization & 50 & 4.5 \\
Polarization & 308 & 27.7 \\
Cusp of minimization & 99 & 8.9 \\
Minimization & 498 & 44.8 \\
Cusp of acceptance & 54 & 4.9 \\
Acceptance & 46 & 4.1 \\
Adaptation & 2 & 0.2 \\
\hline Total & 1,112 & 100 \\
\hline
\end{tabular}




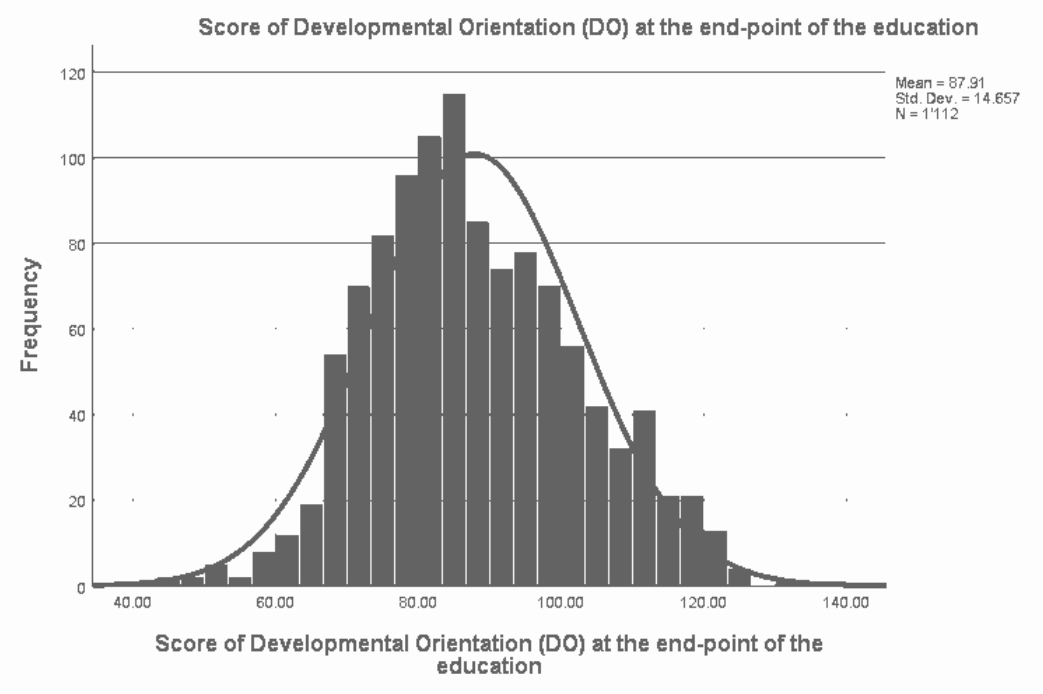

Figure 11. Distribution of the students' score of developmental orientation at the endpoint of the education.

Regardless of the time-point, the Tables 7-10 and Figures 8-11 demonstrate a significant difference between the PO $(M=118.5$ at the start-point; $M=119.3$ at the endpoint of education) and the DO $(M=85.5$ at the start-point, $M=87.9$ at the end-point of education). While the PO represented as lowest mindset the minimization, for the DO the mindset lowest mindset is Denial. This difference between where the nursing students perceive they are on the developmental continuum (PO) and where the IDI places their level of intercultural competence (DO) is called the orientation gap and is presented in Table 11. 
Table 11

Orientation Gap Between Perceived Orientation and Developmental Orientation at Startand End-Point of the Education

\begin{tabular}{lccc}
\hline & $\begin{array}{c}\text { Orientation gap } \\
\text { at the start-point } \\
\text { of the education }\end{array}$ & $\begin{array}{c}\text { Orientation gap } \\
\text { at the end-point } \\
\text { of the education }\end{array}$ & $\begin{array}{l}\text { Orientation gap at the } \\
\text { start-point and the end- } \\
\text { point of the education }\end{array}$ \\
\hline$N$ & 2,284 & 1,126 & 3,410 \\
Mean & 33.1 & 31.3 & 32.5 \\
Median & 33.8 & 32.4 & 33.5 \\
Mode & 39.8 & $42.86 \mathrm{a}$ & 33.8 \\
$S D$ & 8.5 & 9.5 & 8.9 \\
Variance & 72.6 & 90.2 & 79.1 \\
Skewness & -0.3 & -0.2 & -0.3 \\
Kurtosis & -0.1 & -0.2 & -0.1 \\
Minimum & 5.4 & 3.7 & 3.7 \\
Maximum & 65.8 & 61.1 & 65.8 \\
\hline
\end{tabular}

Note. a Multiple modes exist. The smallest value is shown

Table 11 shows a mean orientation gap between 31.3 and 33.1. A gap score of 7 points or higher can be considered a meaningful difference between where the nursing students perceive they are on the developmental continuum and where the IDI places their level of intercultural competence (IDI, 2018b). The negative skewness values between -0.2 and -0.3 indicate a clustering of scores at the high end (Pallant, 2016). The kurtosis value below zero indicates a distribution that is relatively flat (too many cases in the extremes; Pallant, 2016). This value is consistent with the values from a minimum orientation gap of 3.7 and a maximum of 65.8 . In summary, the orientation gap considerably high

\section{Results to Research Question 2}

RQ 2 What is the relationship among the nursing students' demographic variables and the nursing students' orientation toward cultural difference and commonality? 
Because the assumptions for multiple regression analysis and ANOVA were not met, a Chi-square test for independence was applied to test the hypotheses to research question 2. According to Frankfort-Nachmias (2014), the Chi-square test for independence is "an inferential statistical technique designed to test for significant relationships between two nominal or ordinal variables organized in a bivariate table" (p. 350). As a nonparametric test, it can be applied to variables measured at a nominal and/or an ordinal level of measurement (Frankfort-Nachmias, 2014). Like all non-parametric statistics, the Chi-square test for independence is robust concerning the distribution of the data. The chi-square test for independence requires no assumptions about the shape of the population distribution from which the sample was drawn. Specifically, it does not require equality of variances among the study groups or homoscedasticity in the data. However, like all inferential techniques, certain assumptions need to be met. Apart from random sampling, the Chi-square test for independence assumes that the variables are measured at an ordinal or nominal level and consist of two or more categorical, independent groups. In addition, the value of each cell should be 5 or more in at least $80 \%$ of the cells (McHugh, 2013). All of these assumptions were met in this study.

According to the result of the Chi-square test for independence as a significance statistic, the null hypothesis can either be rejected or not. The conclusion can be made whether there is a relationship between the two variables or not. However, the Chi-square test for independence does not provide how strong this relationship is. The Chi-square test should be followed by a strength statistic (McHugh, 2013). Therefore, the Phi test and the Cramer's V test were performed. As a correlation-coefficient, the Phi and 
Cramer's V indicates the size of the effect with a higher value indicating a stronger association between the two variables (Pallant, 2016). The Cramer's V is the most common strength test used to test the data when a significant Chi-square test-result has been obtained (McHugh, 2013).

The results of the chi-square test for independence will be described for the PO and the DO toward cultural difference and commonality being measured within the nursing students' either at the start-point or the end-point of their education. These orientations are represented as a particular mindset on IDC being either denial, cusp of polarization, polarization, curs of minimization, minimization, cusp of acceptance, acceptance, cusp of adaptation, or adaptation. If the assumption that the value of each cell should be 5 or more in at least $80 \%$ of the cells was violated, the mindsets cusp of adaptation and adaptation were merged to one mindset (cusp of) adaptation, as only a few students demonstrated such a mindset.

\section{Relationship Between the Students' Age and Their PO}

The variable students' age previously had the levels 17 and under, 18-21, 22-30, $31--40,41-50,51-60$, and 61 and over. Because the assumption that the value of each cell should be 5 or more in at least $80 \%$ of the cells was violated, the levels 17 and under and $18-21$ were merged to one level named $17-21$. For the same reason, the levels $22-30$ and 31-40 were merged to one level named 22-40, and the levels 41-50, 51-60, and 61 and over were merged to one level named 41 and over. 
Table 12

Observed and Expected Frequency of the Perceived Orientation for Age

\begin{tabular}{|c|c|c|c|c|c|}
\hline & & $17-21$ & $22-40$ & 41 and over & Total \\
\hline \multirow[t]{2}{*}{ Minimization } & Count & 53 & 40 & 6 & 99 \\
\hline & Expected Count & 48.9 & 45.3 & 4.8 & 99 \\
\hline \multirow{2}{*}{ Cusp of Acceptance } & Count & 396 & 286 & 20 & 702 \\
\hline & Expected Count & 346.5 & 321.3 & 34.2 & 702 \\
\hline \multirow[t]{2}{*}{ Acceptance } & Count & 1,148 & 1,099 & 119 & 2,366 \\
\hline & Expected Count & 1,168 & 1,083 & 115 & 2,366 \\
\hline \multicolumn{6}{|l|}{ (Cusp of) } \\
\hline Adaptation & Expected Count & 118 & 109.4 & 11.6 & 239 \\
\hline \multirow[t]{2}{*}{ Total } & Count & 1,681 & 1,559 & 166 & 3,406 \\
\hline & Expected Count & 1,681 & 1,559 & 166 & 3,406 \\
\hline
\end{tabular}

The observed (counted) and the expected frequency of the PO for the students' age are different (Table 12). A chi-square test of independence was performed to examine the relation between the students' PO and the students' age. The relation between the students' PO and their age was significant, $X^{2}(6, N=3,406)=41.64, p<.001$. The null hypothesis that there is no relationship between the students' age and their stage on the IDC can be rejected. It can be concluded that the students' PO is dependent on the students' age. However, the value of Phi $=.11$ and Cramer's V $=.08$ indicate a small effect size.

\section{Relationship Between the Students' Age and Their Developmental Orientation}

The same merged levels for the students' age were used for the DO as described above for the analysis of the PO. 
Table 13

Observed and Expected Frequency of Developmental Orientation for Students' Age

\begin{tabular}{llllll}
\hline \multirow{4}{*}{ Denial } & & $17-21$ & $22-40$ & 41 and over & Total \\
Cusp of polarization & Count & 118 & 73 & 8 & 199 \\
\cline { 2 - 6 } Polarization & Expected count & 98.2 & 91.1 & 9.7 & 199 \\
\cline { 2 - 6 } & Count & 77 & 68 & 7 & 152 \\
\cline { 2 - 6 } Cusp of minimization & Expected count & 75 & 69.6 & 7.4 & 152 \\
\cline { 2 - 6 } & Count & 575 & 440 & 26 & 1,041 \\
\cline { 2 - 6 } Minimization & Expected count & 514 & 476.5 & 50.7 & 1,041 \\
\cline { 2 - 6 } & Count & 152 & 159 & 18 & 329 \\
\cline { 2 - 6 } Cusp of acceptance & Expected count & 162 & 150.6 & 16 & 329 \\
\cline { 2 - 6 } Acceptance & Count & 696 & 707 & 95 & 1,498 \\
\cline { 2 - 6 } & Expected count & 739 & 685.7 & 73 & 1,498 \\
\cline { 2 - 6 } (Cusp of) adaptation & Count & 38 & 55 & 7 & 100 \\
\cline { 2 - 6 } & Expected count & 49.4 & 45.8 & 4.9 & 100 \\
\cline { 2 - 6 } Total & Count & 24 & 53 & 5 & 82 \\
\cline { 2 - 6 } & Expected count & 40.5 & 37.5 & 4 & 82 \\
\cline { 2 - 6 } & Count & 1 & 4 & 0 & 5 \\
\cline { 2 - 6 } & Expected count & 2.5 & 2.3 & 0.2 & 5 \\
\cline { 2 - 6 } & Count & 1,681 & 1,559 & 166 & 3,406 \\
\cline { 2 - 5 } & Expected count & 1,681 & 1,559 & 166 & 306 \\
\hline
\end{tabular}

The observed (counted) and the expected frequency of the DO for the students' age are different (Table 13). A chi-square test of independence was performed to examine the relation between the students' DO and their age. The relation between the students' DO and their age was significant, $X^{2}(14, N=3,406)=62.46, p<.001$. The null hypothesis that there is no relationship between the students' age and their stage on the IDC can be rejected. It can be concluded that the students' DO is dependent on the students' age. However, the values of Phi $=.14$ and Cramer's V $=.10$ indicate a small effect size. 


\section{Relationship Between the Students' Gender and Their Perceived Orientation}

The variable students' gender has two levels: female and male.

Table 14

Observed and Expected Frequency of the Perceived Orientation for Students' Gender

\begin{tabular}{lllll}
\hline \multirow{4}{*}{ Minimization } & & Male & Female & Total \\
\cline { 2 - 5 } Cusp of acceptance & Count & 41 & 57 & 98 \\
\cline { 2 - 5 } Expected count & 35.6 & 62.4 & 98 \\
\cline { 2 - 5 } Acceptance & Count & 283 & 408 & 691 \\
\cline { 2 - 5 } Cusp of adaptation count & 251 & 440 & 691 \\
\cline { 2 - 5 } & Count & 807 & 1531 & 2,338 \\
\cline { 2 - 5 } Adaptation & Expected count & 849.1 & $1,488.9$ & 2,338 \\
\cline { 2 - 5 } & Count & 66 & 98 & 164 \\
\cline { 2 - 5 } Total & Expected count & 59.6 & 104.4 & 164 \\
\cline { 2 - 5 } & Count & 24 & 47 & 71 \\
\cline { 2 - 5 } & Expected count & 25.8 & 45.2 & 71 \\
\cline { 2 - 5 } & Expected count & 1,221 & 2,141 & 3,362 \\
\hline
\end{tabular}

The observed (counted) and the expected frequency of the PO for the students' gender are different (Table 14). A chi-square test of independence was performed to examine the relation between the students' PO and their gender. The relation between the students' PO and their gender was significant, $X^{2}(4, N=3,362)=12.28, p=.02$. The null hypothesis that there is no relationship between the students' gender and their stage on the IDC can be rejected. It can be concluded that the students' PO is dependent on their gender. However, the values of Phi $=.06$ and Cramer's V $=.06$ indicate a small effect size.

\section{Relationship Between the Students' Gender and Their Developmental Orientation}

The variable students' gender has two levels: female and male. 
Table 15

Observed and Expected Frequency of the Developmental Orientation for Students' Gender

\begin{tabular}{|c|c|c|c|c|}
\hline & & Male & Female & Total \\
\hline \multirow[t]{2}{*}{ Denial } & Count & 85 & 110 & 195 \\
\hline & Expected count & 70.8 & 124.2 & 195 \\
\hline \multirow[t]{2}{*}{ Cusp of polarization } & Count & 71 & 80 & 151 \\
\hline & Expected count & 54.8 & 96.2 & 151 \\
\hline \multirow[t]{2}{*}{ Polarization } & Count & 359 & 670 & 1,029 \\
\hline & Expected count & 373.7 & 655.3 & 1,029 \\
\hline \multirow[t]{2}{*}{ Cusp of minimization } & Count & 108 & 219 & 327 \\
\hline & Expected count & 118.8 & 208.2 & 327 \\
\hline \multirow[t]{2}{*}{ Minimization } & Count & 519 & 959 & 1,478 \\
\hline & Expected count & 536.8 & 941.2 & 1,478 \\
\hline \multirow[t]{2}{*}{ Cusp of acceptance } & Count & 46 & 52 & 98 \\
\hline & Expected count & 35.6 & 62.4 & 98 \\
\hline \multirow[t]{2}{*}{ Acceptance } & Count & 32 & 47 & 79 \\
\hline & Expected count & 28.7 & 50.3 & 79 \\
\hline \multirow[t]{2}{*}{ Cusp of adaptation } & Count & 0 & 2 & 2 \\
\hline & Expected count & 0.7 & 1.3 & 2 \\
\hline \multirow[t]{2}{*}{ Adaptation } & Count & 1 & 2 & 3 \\
\hline & Expected count & 1.1 & 1.9 & 3 \\
\hline \multirow[t]{2}{*}{ Total } & Count & 1,221 & 2,141 & 3,362 \\
\hline & Expected count & 1,221 & 2,141 & 3,362 \\
\hline
\end{tabular}

The observed (counted) and the expected frequency of the DO for the students' gender are different (Table 15). A chi-square test of independence was performed to examine the relation between the students' DO and their gender. The relation between the students' DO and their gender was significant, $X^{2}(8, N=3,362)=21.83, p=.01$. The null hypothesis that there is no relationship between the students' gender and their stage on the IDC can be rejected. It can be concluded that the students' DO is dependent on their gender. However, the values of Phi $=.08$ and Cramer's V $=.08$ indicate a small effect size. 


\section{Relationship Between the Students' Primary Country of Citizenship and Their Perceived Orientation}

The variable students' primary country of citizenship has the two levels: Swiss citizenship and citizenship of another country.

Table 16

Observed and Expected Frequency of Perceived Orientation for Students' Primary Country of Citizenship

\begin{tabular}{lllll}
\hline \multirow{2}{*}{ Minimization } & \multicolumn{1}{c}{$\begin{array}{l}\text { Swiss } \\
\text { Citizenship }\end{array}$} & $\begin{array}{l}\text { Citizenship of } \\
\text { another country }\end{array}$ & Total \\
\hline \multirow{4}{*}{ Cusp of acceptance } & Count & 86 & 13 & 99 \\
\cline { 2 - 5 } Acceptance & Expected count & 89.3 & 9.7 & 99 \\
\cline { 2 - 5 } & Count & 632 & 65 & 697 \\
\cline { 2 - 5 } (Cusp of) adaptation & Expected count & 628.5 & 68.5 & 697 \\
\cline { 2 - 5 } & Count & 2,141 & 218 & 2,359 \\
\cline { 2 - 5 } Total & Expected count & 2,127 & 231.7 & 2,359 \\
\cline { 2 - 5 } & Count & 198 & 37 & 235 \\
\cline { 2 - 5 } & Expected count & 211.9 & 23.1 & 3,390 \\
\cline { 2 - 5 } & Count & 3,057 & 333 & 3390 \\
\cline { 2 - 5 } & Expected count & 3,057 & 333 & \\
\hline
\end{tabular}

The observed (counted) and the expected frequency of the PO for the students' primary country of citizenship are different (Table 16). A chi-square test of independence was performed to examine the relation between the students' PO and their primary country of citizenship. The relation between the students' PO and their primary country of citizenship was significant, $X^{2}(3, N=3,390)=11.62, p=.01$. The null hypothesis that there is no relationship between the students' primary country of citizenship and their stage on the IDC can be rejected. It can be concluded that the students' PO is dependent 
on their primary country of citizenship. However, the values of Phi $=.06$ and Cramer's V $=.06$ indicate a small effect size.

\section{Relationship Between the Students' Primary Country of Citizenship and Their Developmental Orientation}

The variable students' primary country of citizenship has the two levels: Swiss citizenship and citizenship of another country.

Table 17

Observed and Expected Frequency of Developmental Orientation for Students' Primary Country of Citizenship

\begin{tabular}{|c|c|c|c|c|}
\hline & & $\begin{array}{l}\text { Swiss } \\
\text { Citizenship }\end{array}$ & $\begin{array}{l}\text { Citizenship of } \\
\text { another country }\end{array}$ & Total \\
\hline \multirow[t]{2}{*}{ Denial } & Count & 178 & 21 & 199 \\
\hline & Expected count & 179.5 & 19.5 & 199 \\
\hline \multirow[t]{2}{*}{ Cusp of polarization } & Count & 128 & 23 & 151 \\
\hline & Expected count & 136.2 & 14.8 & 151 \\
\hline \multirow[t]{2}{*}{ Polarization } & Count & 947 & 89 & 1,036 \\
\hline & Expected count & 934.2 & 101.8 & 1,036 \\
\hline \multirow[t]{2}{*}{ Cusp of minimization } & Count & 296 & 31 & 327 \\
\hline & Expected count & 294.9 & 32.1 & 327 \\
\hline \multirow[t]{2}{*}{ Minimization } & Count & 1353 & 141 & 1,494 \\
\hline & Expected count & $1,347.2$ & 146.8 & 1,494 \\
\hline \multirow[t]{2}{*}{ Cusp of acceptance } & Count & 87 & 12 & 99 \\
\hline & Expected count & 89.3 & 9.7 & 99 \\
\hline \multirow[t]{2}{*}{ Acceptance } & Count & 63 & 16 & 79 \\
\hline & Expected count & 71.2 & 7.8 & 79 \\
\hline \multirow[t]{2}{*}{ (Cusp of) adaptation } & Count & 5 & 0 & 5 \\
\hline & Expected count & 4.5 & 0.5 & 5 \\
\hline \multirow[t]{2}{*}{ Total } & Count & 3,057 & 333 & 3,390 \\
\hline & Expected count & 3,057 & 333 & 3,390 \\
\hline
\end{tabular}


The observed (counted) and the expected frequency of the DO for the students' primary country of citizenship are different (Table 17). A chi-square test of independence was performed to examine the relation between the students' DO and their primary country of citizenship. The relation between the students' DO and their primary country of citizenship was significant, $X^{2}(7, N=3,390)=18.01, p=.01$. The null hypothesis that there is no relationship between the students' primary country of citizenship and their stage on the IDC can be rejected. It can be concluded that the students' DO is dependent on their primary country of citizenship. However, the values of Phi $=.07$ and Cramer's V $=.07$ indicate a small effect size.

\section{Relationship Between the Students' Ethnic Minority Group Affiliation and Their Perceived Orientation}

The variable students' ethnic minority group affiliation has two levels: yes and no. 
Table 18

Observed and Expected Frequency of Perceived Orientation for Students' Ethnic Minority Group Affiliation

\begin{tabular}{lllll}
\hline \multirow{3}{*}{ Minimization } & & Yes & No & Total \\
\cline { 2 - 5 } Cusp of acceptance & Count & 5 & 72 & 77 \\
\cline { 2 - 5 } Acceptance & Expected count & 5.7 & 71.3 & 77 \\
\cline { 2 - 5 } & Count & 48 & 553 & 601 \\
\cline { 2 - 5 } Cusp of adaptation & Expected count & 44.6 & 556.4 & 601 \\
\cline { 2 - 5 } & Count & 138 & 1,772 & 1,910 \\
\cline { 2 - 5 } Adaptation & Expected count & 141.6 & 1,768 & 1,910 \\
\cline { 2 - 5 } & Count & 13 & 130 & 143 \\
\cline { 2 - 5 } Total & Expected count & 10.6 & 132.4 & 143 \\
\cline { 2 - 5 } & Count & 2.5 & 58 & 61 \\
\cline { 2 - 5 } & Expected count & 207 & 2,585 & 2,792 \\
\cline { 2 - 5 } & Count & & 2,585 & 2,792 \\
\cline { 2 - 5 } & Expected count & 207 & \\
\hline
\end{tabular}

The observed (counted) and the expected frequency of the PO for the students' ethnic minority group affiliation are similar (Table 18). A chi-square test of independence was performed to examine the relation between the students' PO and their ethnic minority group affiliation. The relation between the students' PO and their ethnic minority group affiliation was not significant, $X^{2}(4, N=2,792)=1.62, p=.81$. The null hypothesis that there is no relationship between the students' ethnic minority group affiliation and their stage on the IDC cannot be rejected. It can be concluded that the students' PO is independent of their ethnic minority group affiliation.

\section{Relationship Between the Students' Ethnic Minority Group Affiliation and Their Developmental Orientation}

The variable students' ethnic minority group affiliation has two levels: yes and no. 
Table 19

Observed and Expected Frequency of the Developmental Orientation for the Students' Ethnic Minority Group Affiliation

\begin{tabular}{|c|c|c|c|c|}
\hline & & Yes & No & Total \\
\hline \multirow[t]{2}{*}{ Denial } & Count & 15 & 157 & 172 \\
\hline & Expected count & 12.8 & 159.2 & 172 \\
\hline \multirow[t]{2}{*}{ Cusp of polarization } & Count & 18 & 109 & 127 \\
\hline & Expected count & 9.4 & 117.6 & 127 \\
\hline \multirow[t]{2}{*}{ Polarization } & Count & 64 & 789 & 853 \\
\hline & Expected count & 63.2 & 789.8 & 853 \\
\hline \multirow[t]{2}{*}{ Cusp of minimization } & Count & 18 & 236 & 254 \\
\hline & Expected count & 18.8 & 235.2 & 254 \\
\hline \multirow[t]{2}{*}{ Minimization } & Count & 79 & 1,138 & 1,217 \\
\hline & Expected count & 90.2 & 1,127 & 1,217 \\
\hline \multirow[t]{2}{*}{ Cusp of acceptance } & Count & 6 & 83 & 89 \\
\hline & Expected count & 6.6 & 82.4 & 89 \\
\hline \multirow[t]{2}{*}{ Acceptance } & Count & 7 & 69 & 76 \\
\hline & Expected count & 5.6 & 70.4 & 76 \\
\hline \multirow[t]{2}{*}{ (Cusp of) adaptation } & Count & 0 & 4 & 4 \\
\hline & Expected count & 0.3 & 3.7 & 4 \\
\hline \multirow[t]{2}{*}{ Total } & Count & 207 & 2,585 & 2,792 \\
\hline & Expected count & 207 & 2,585 & 2,792 \\
\hline
\end{tabular}

The observed (counted) and the expected frequency of the DO for the students' ethnic minority group affiliation are similar (Table 19). A chi-square test of independence was performed to examine the relation between the students' DO and their ethnic minority group affiliation. The relation between the students DO and their ethnic minority group affiliation was not significant, $X^{2}(7, N=2792)=11.18, p=.13$. The null hypothesis that there is no relationship between the students' ethnic minority group affiliation and their stage on the IDC cannot be rejected. It can be concluded that the students' DO is independent of their ethnic minority group affiliation. 


\section{Relationship Between the Students' Migration Background and Their Perceived}

\section{Orientation}

The variable students' migration background has the two levels: yes and no.

Table 20

Observed and Expected Frequency of Perceived Orientation for Students' Migration Background

\begin{tabular}{lllll}
\hline \multirow{4}{*}{ Minimization } & & Yes & No & Total \\
\cline { 2 - 5 } Cusp of acceptance & Count & 17 & 79 & 96 \\
\cline { 2 - 5 } Acceptance & Expected count & 16.5 & 79.5 & 96 \\
\cline { 2 - 5 } & Count & 113 & 567 & 680 \\
\cline { 2 - 5 } (Cusp of) adaptation & Expected count & 116.7 & 563.3 & 680 \\
\cline { 2 - 5 } & Count & 392 & 1,925 & 2,317 \\
\cline { 2 - 5 } Total & Expected count & 397.5 & $1,919.5$ & 2,317 \\
\cline { 2 - 5 } & Count & 49 & 186 & 235 \\
\cline { 2 - 5 } & Expected count & 40.3 & 194.7 & 235 \\
\cline { 2 - 5 } & Count & 571 & 2,757 & 3,328 \\
\cline { 2 - 5 } & Expected count & 571 & 2,757 & 3,328 \\
\hline
\end{tabular}

The observed (counted) and the expected frequency of the PO for the students' migration background are similar (Table 20). A chi-square test of independence was performed to examine the relation between the students' PO and their migration background. The relation between the students' PO and their migration background was not significant, $X^{2}(3, N=3,328)=2.51, p=.47$. The null hypothesis that there is no relationship between the students' migration background and their stage on the IDC cannot be rejected. It can be concluded that the students' PO is independent of their migration background. 


\section{Relationship Between the Students' Migration Background and Their}

\section{Developmental Orientation}

The variable students' migration background has the two levels: yes and no.

Table 21

Observed and Expected Frequency of Developmental Orientation for Students' Migration Background

\begin{tabular}{|c|c|c|c|c|}
\hline & & Yes & No & Total \\
\hline \multirow[t]{2}{*}{ Denial } & Count & 40 & 154 & 194 \\
\hline & Expected count & 33.3 & 160.7 & 194 \\
\hline \multirow{2}{*}{ Cusp of polarization } & Count & 32 & 114 & 146 \\
\hline & Expected count & 25 & 121 & 146 \\
\hline \multirow[t]{2}{*}{ Polarization } & Count & 153 & 859 & 1,012 \\
\hline & Expected count & 173.6 & 838.4 & 1,012 \\
\hline \multirow{2}{*}{ Cusp of minimization } & Count & 70 & 252 & 322 \\
\hline & Expected count & 55.2 & 266.8 & 322 \\
\hline \multirow[t]{2}{*}{ Minimization } & Count & 239 & 1,231 & 1,470 \\
\hline & Expected count & 252.2 & $1,217.8$ & 1,470 \\
\hline \multirow{2}{*}{ Cusp of acceptance } & Count & 19 & 80 & 99 \\
\hline & Expected count & 17 & 82 & 99 \\
\hline \multirow[t]{2}{*}{ Acceptance } & Count & 17 & 63 & 80 \\
\hline & Expected count & 13.7 & 66.3 & 80 \\
\hline \multirow[t]{2}{*}{ (Cusp of) adaptation } & Count & 1 & 4 & 5 \\
\hline & Expected count & 0.9 & 4.1 & 5 \\
\hline \multirow[t]{2}{*}{ Total } & Count & 571 & 2,757 & 3,328 \\
\hline & Expected count & 571 & 2,757 & 3,328 \\
\hline
\end{tabular}

The observed (counted) and the expected frequency of the DO for the students' migration background are similar (Table 21). A chi-square test of independence was performed to examine the relation between the students DO and their migration background. The relation between the students DO and the students' previous education as health care assistant was not significant, $X^{2}(7, N=3,328)=13.77, p=.06$. The null 
hypothesis that there is no relationship between the students' migration background and their stage on the IDC cannot be rejected. It can be concluded that the students' DO is independent of their migration background.

\section{Relationship Between the World Region Students Primarily Lived Up to Age 18 and}

\section{Their Perceived Orientation}

The world region students primarily lived up to age 18 previously had the levels North America, Central America, South America, Middle East, Africa, Australia, Asia Pacific, Western Europe, and Eastern Europe. Because the assumption that the value of each cell should be five or more in at least $80 \%$ of the cells was violated, the levels North America, Central America, South America, Middle East, Africa, Australia, and Asia Pacific were merged to one level named Other World Regions.

Table 22

Observed and Expected Frequency of Perceived Orientation for World Region Students Primarily Lived up to Age 18

\begin{tabular}{llllll}
\hline \multirow{2}{*}{ Minimization } & & $\begin{array}{l}\text { Western } \\
\text { Europe }\end{array}$ & $\begin{array}{l}\text { Eastern } \\
\text { Europe }\end{array}$ & $\begin{array}{l}\text { Other World } \\
\text { Regions }\end{array}$ & Total \\
\hline \multirow{3}{*}{ Cusp of acceptance } & Count & 85 & 6 & 8 & 99 \\
\cline { 2 - 6 } Acceptance & Expected count & 88.4 & 4.1 & 6.5 & 99 \\
\cline { 2 - 6 } & Count & 613 & 25 & 61 & 699 \\
\cline { 2 - 6 } (Cusp of) adaptation & Expected count & 623.9 & 29.2 & 45.9 & 699 \\
\cline { 2 - 6 } & Count & 2,128 & 95 & 140 & 2,363 \\
\cline { 2 - 6 } Total & Expected count & $2,109.3$ & 98.7 & 155 & 2,363 \\
\cline { 2 - 6 } & Count & 208 & 16 & 14 & 238 \\
\cline { 2 - 6 } & Expected count & 212.4 & 9.9 & 15.6 & 238 \\
\cline { 2 - 6 } & Count & 3,034 & 142 & 223 & 3,399 \\
\cline { 2 - 6 } & Expected count & 3,034 & 142 & 223 & 3,399 \\
\hline
\end{tabular}


The observed (counted) and the expected frequency of the PO for the world region students primarily lived up to age 18 are different (Table 22). A chi-square test of independence was performed to examine the relation between the students' PO and the world region students primarily lived up to age 18 . The relation between the students' PO and the world region students primarily lived up to age 18 was not significant, $X^{2}(6, N=$ $3399)=12.83, p=.05$. The null hypothesis that there is no relationship between the world region students primarily lived up to age 18 and the students' stage on the IDC cannot be rejected. It can be concluded that the students' PO is independent of the world region students primarily lived up to age 18 .

\section{Relationship Between the World Region Students Primarily Lived Up to Age 18 and Their Developmental Orientation}

The world region students primarily lived up to age 18 previously had the levels North America, Central America, South America, Middle East, Africa, Australia, Asia Pacific, Western Europe, and Eastern Europe. Because the assumption that the value of each cell should be five or more in at least $80 \%$ of the cells was violated, the levels North America, Central America, South America, Middle East, Africa, Australia, and Asia Pacific were merged to one level named Other World Regions.

Table 23 
Observed and Expected Frequency of Developmental Orientation for World Regions Students Primarily Lived up to Age 18

\begin{tabular}{|c|c|c|c|c|c|}
\hline & & $\begin{array}{l}\text { Western } \\
\text { Europe }\end{array}$ & $\begin{array}{l}\text { Eastern } \\
\text { Europe }\end{array}$ & $\begin{array}{l}\text { Other World } \\
\text { Regions }\end{array}$ & Total \\
\hline \multirow[t]{2}{*}{ Denial } & Count & 169 & 8 & 21 & 198 \\
\hline & Expected count & 176.7 & 8.3 & 13 & 198 \\
\hline \multirow[t]{2}{*}{ Cusp of polarization } & Count & 131 & 11 & 10 & 152 \\
\hline & Expected count & 135.7 & 6.4 & 10 & 152 \\
\hline \multirow[t]{2}{*}{ Polarization } & Count & 929 & 45 & 67 & 1,041 \\
\hline & Expected count & 929.2 & 43.5 & 68.3 & 1,041 \\
\hline \multirow[t]{2}{*}{ Cusp of minimization } & Count & 290 & 15 & 22 & 327 \\
\hline & Expected count & 291.9 & 13.7 & 21.5 & 327 \\
\hline \multirow[t]{2}{*}{ Minimization } & Count & 1,351 & 52 & 92 & 1,495 \\
\hline & Expected count & $1,334.5$ & 62.5 & 98.1 & 1,495 \\
\hline \multirow[t]{2}{*}{ Cusp of acceptance } & Count & 92 & 2 & 5 & 99 \\
\hline & Expected count & 88.4 & 4.1 & 6.5 & 99 \\
\hline \multirow[t]{2}{*}{ Acceptance } & Count & 67 & 9 & 6 & 82 \\
\hline & Expected count & 73.2 & 3.4 & 5.4 & 82 \\
\hline \multirow[t]{2}{*}{ (Cusp of) adaptation } & Count & 5 & 0 & 0 & 5 \\
\hline & Expected count & 4.5 & 0.2 & 0.3 & 5 \\
\hline \multirow[t]{2}{*}{ Total } & Count & 3,034 & 142 & 223 & 3,399 \\
\hline & Expected count & 3,034 & 142 & 223 & 3,399 \\
\hline
\end{tabular}

The observed (counted) and the expected frequency of the DO for the world region students primarily lived up to age 18 are similar (Table 23). A chi-square test of independence was performed to examine the relation between the students DO and the world region students primarily lived up to age 18 . The relation between the students' DO and the world region students primarily lived up to age 18 was not significant, $X^{2}$ (14, $N=3,399)=23.28, p=.06$. The null hypothesis that there is no relationship between the world region students primarily lived up to age 18 and the students' stage on the IDC cannot be rejected. It can be concluded that the students' DO is independent of the world region students primarily lived up to age 18 . 


\section{Relationship Between the Total Amount of Time Students Lived in Another}

\section{Country and Their Perceived Orientation}

The variable total amount of time students lived in another country has the eight levels never lived in another country, less than 3 months, 3-6 month, 7-11 months, 1-2 years, 3-5 years, $6-10$ years, and over 10 years.

Table 24

Observed and Expected Frequency of Perceived Orientation for Time Students Lived in Another Country

\begin{tabular}{|c|c|c|c|c|c|c|c|c|c|c|}
\hline & & $\begin{array}{l}\text { never } \\
\text { lived in } \\
\text { another } \\
\text { country }\end{array}$ & $\begin{array}{l}\text { less } \\
\text { than } 3 \\
\text { months }\end{array}$ & $\begin{array}{l}3-6 \\
\text { months }\end{array}$ & $\begin{array}{l}7-11 \\
\text { months }\end{array}$ & $\begin{array}{l}1-2 \\
\text { years }\end{array}$ & $\begin{array}{l}3-5 \\
\text { years }\end{array}$ & $\begin{array}{l}6-10 \\
\text { years }\end{array}$ & $\begin{array}{l}\text { over } \\
10 \\
\text { years }\end{array}$ & Total \\
\hline \multirow[t]{2}{*}{ Minimization } & Count & 49 & 20 & 16 & 2 & 4 & 2 & 1 & 5 & 99 \\
\hline & $\begin{array}{l}\text { Expected } \\
\text { count }\end{array}$ & 44 & 17.2 & 14.9 & 6.5 & 6.9 & 3.1 & 2 & 4.5 & 99 \\
\hline \multirow[t]{2}{*}{$\begin{array}{l}\text { Cusp of } \\
\text { acceptance }\end{array}$} & Count & 328 & 130 & 114 & 36 & 35 & 21 & 9 & 23 & 696 \\
\hline & $\begin{array}{l}\text { Expected } \\
\text { count }\end{array}$ & 309 & 120.9 & 104.5 & 45.9 & 48 & 22 & 14.1 & 31.4 & 696 \\
\hline \multirow[t]{2}{*}{ Acceptance } & Count & 1,035 & 412 & 341 & 172 & 170 & 76 & 53 & 104 & 2,363 \\
\hline & $\begin{array}{l}\text { Expected } \\
\text { count }\end{array}$ & 1,049 & 410.5 & 354.9 & 156 & 164 & 75 & 48 & 106.5 & 2,363 \\
\hline \multirow[t]{2}{*}{$\begin{array}{l}\text { (Cusp of) } \\
\text { adaptation }\end{array}$} & Count & 96 & 28 & 39 & 14 & 26 & 8 & 6 & 21 & 238 \\
\hline & $\begin{array}{l}\text { Expected } \\
\text { count }\end{array}$ & 106 & 41.3 & 35.7 & 15.7 & 17 & 7.5 & 4.8 & 10.7 & 238 \\
\hline \multirow[t]{2}{*}{ Total } & Count & 1,508 & 590 & 510 & 224 & 235 & 107 & 69 & 153 & 3,396 \\
\hline & $\begin{array}{l}\text { Expected } \\
\text { count }\end{array}$ & 1,508 & 590 & 510 & 224 & 235 & 107 & 69 & 153 & 3,396 \\
\hline
\end{tabular}

The observed (counted) and the expected frequency of the PO for the total amount of time students lived in another country are different (Table 24). A chi-square test of independence was performed to examine the relation between the students' PO and total amount of time students lived in another country. The relation between the students' PO 
and the total amount of time students lived in another country was significant, $X^{2}(21, N=$ $3,396)=43.63, p=.003$. The null hypothesis that there is no relationship between the total amount of time students lived in another country and the students' stage on the IDC can be rejected. It can be concluded that the students' PO is dependent on the total amount of time students lived in another country. However, the value of Phi $=.11$ and Cramer's $\mathrm{V}=.07$ indicate a small effect size.

\section{Relationship Between the Total Amount of Time Students Lived in Another \\ Country and Their Developmental Orientation}

The variable total amount of time students lived in another country has the eight levels never lived in another country, less than 3 months, 3-6 month, 7-11 months, 1-2 years, 3-5 years, 6-10 years, and over 10 years.

Table 25 
Observed and Expected Frequency of Developmental Orientation for Time Students Lived in Another Country

\begin{tabular}{|c|c|c|c|c|c|c|c|c|c|c|}
\hline & & $\begin{array}{l}\text { never lived } \\
\text { in another } \\
\text { country }\end{array}$ & $\begin{array}{l}\text { less } \\
\text { than } 3 \\
\text { months }\end{array}$ & $\begin{array}{l}3-6 \\
\text { months }\end{array}$ & $\begin{array}{l}7-11 \\
\text { months }\end{array}$ & $\begin{array}{l}1-2 \\
\text { years }\end{array}$ & $\begin{array}{l}3-5 \\
\text { years }\end{array}$ & $\begin{array}{l}6-10 \\
\text { years }\end{array}$ & $\begin{array}{l}\text { over } \\
10 \\
\text { years }\end{array}$ & Total \\
\hline \multirow[t]{2}{*}{ Denial } & Count & 94 & 37 & 32 & 8 & 7 & 8 & 3 & 8 & 197 \\
\hline & $\begin{array}{l}\text { Expected } \\
\text { count }\end{array}$ & 87.5 & 34.2 & 29.6 & 13 & 13.6 & 6.2 & 4 & 8.9 & 197 \\
\hline \multirow{2}{*}{$\begin{array}{l}\text { Cusp of } \\
\text { polarization }\end{array}$} & Count & 71 & 25 & 20 & 10 & 13 & 5 & 1 & 5 & 150 \\
\hline & $\begin{array}{l}\text { Expected } \\
\text { count }\end{array}$ & 66.6 & 26.1 & 22.5 & 9.9 & 10.4 & 4.7 & 3 & 6.8 & 150 \\
\hline \multirow[t]{2}{*}{ Polarization } & Count & 458 & 218 & 150 & 73 & 66 & 25 & 17 & 34 & 1,041 \\
\hline & $\begin{array}{l}\text { Expected } \\
\text { count }\end{array}$ & 462.3 & 180.9 & 156 & 68.7 & 72 & 32.8 & 21 & 47 & 1,041 \\
\hline \multirow{2}{*}{$\begin{array}{l}\text { Cusp of } \\
\text { Minimization }\end{array}$} & Count & 138 & 59 & 48 & 26 & 18 & 12 & 11 & 15 & 327 \\
\hline & $\begin{array}{l}\text { Expected } \\
\text { count }\end{array}$ & 145.2 & 56.8 & 49.1 & 21.6 & 22.6 & 10.3 & 6.6 & 15 & 327 \\
\hline \multirow[t]{2}{*}{ Minimization } & Count & 666 & 229 & 230 & 97 & 113 & 52 & 32 & 76 & 1,495 \\
\hline & $\begin{array}{l}\text { Expected } \\
\text { count }\end{array}$ & 663.9 & 259.7 & 225 & 98.6 & 103.5 & 47.1 & 30 & 67 & 1,495 \\
\hline \multirow{2}{*}{$\begin{array}{l}\text { Cusp of } \\
\text { acceptance }\end{array}$} & Count & 49 & 14 & 14 & 4 & 10 & 2 & 2 & 4 & 99 \\
\hline & $\begin{array}{l}\text { Expected } \\
\text { count }\end{array}$ & 44 & 17.2 & 14.9 & 6.5 & 6.9 & 3.1 & 2 & 4.5 & 99 \\
\hline \multirow[t]{2}{*}{ Acceptance } & Count & 31 & 7 & 16 & 5 & 6 & 3 & 3 & 11 & 82 \\
\hline & $\begin{array}{l}\text { Expected } \\
\text { count }\end{array}$ & 36.4 & 14.2 & 12.3 & 5.4 & 5.7 & 2.6 & 1.7 & 3.7 & 82 \\
\hline \multirow{2}{*}{$\begin{array}{l}\text { (Cusp of) } \\
\text { adaptation }\end{array}$} & Count & 1 & 1 & 0 & 1 & 2 & 0 & 0 & 0 & 5 \\
\hline & $\begin{array}{l}\text { Expected } \\
\text { count }\end{array}$ & 2.2 & 0.9 & 0.8 & 0.3 & 0.3 & 0.2 & 0.1 & 0.2 & 5 \\
\hline \multirow[t]{2}{*}{ Total } & Count & 1,508 & 590 & 510 & 224 & 235 & 107 & 69 & 153 & 3,396 \\
\hline & $\begin{array}{l}\text { Expected } \\
\text { count }\end{array}$ & 1,508 & 590 & 510 & 224 & 235 & 107 & 69 & 153 & 3,396 \\
\hline
\end{tabular}

The observed (counted) and the expected frequency of the DO for the total amount of time students lived in another country are different (Table 25). A chi-square test of independence was performed to examine the relation between the students DO and the total amount of time students lived in another country. The relation between the students' DO and the total amount of time students lived in another country was 
significant, $X^{2}(49, N=3,396)=73.33, p=.01$. The null hypothesis that there is no relationship between the total amount of time students lived in another country and the students' stage on the IDC can be rejected. It can be concluded that the students' DO is dependent on the total amount of time students lived in another country. However, the value of Phi $=.15$ and Cramer's V $=.06$ indicate a small effect size.

\section{Relationship Between the Amount of Lessons About Own Culture or Any Foreign}

\section{Cultures the Students Had and Their Perceived Orientation}

The variable amount of lessons about own culture or any foreign cultures the students had has the six levels no, less than 1 day, less than 3 days, less than 1 week, less than 2 weeks, and more than 2 weeks.

Table 26

Observed and Expected Frequency of Perceived Orientation for Amount of Lessons on Culture

\begin{tabular}{|c|c|c|c|c|c|c|c|c|}
\hline & & no & $\begin{array}{l}\text { less } \\
\text { than } 1 \\
\text { day }\end{array}$ & $\begin{array}{l}\text { less } \\
\text { than } 3 \\
\text { days }\end{array}$ & $\begin{array}{l}\text { less } \\
\text { than } 1 \\
\text { week }\end{array}$ & $\begin{array}{l}\text { less } \\
\text { than } 2 \\
\text { weeks }\end{array}$ & $\begin{array}{l}\text { more } \\
\text { than } 2 \\
\text { weeks }\end{array}$ & Total \\
\hline \multirow[t]{2}{*}{ Minimization } & Count & 36 & 8 & 18 & 15 & 3 & 19 & 99 \\
\hline & Expected count & 30.1 & 8 & 16.8 & 14.7 & 7.9 & 21.5 & 99 \\
\hline \multirow[t]{2}{*}{ Cusp of acceptance } & Count & 217 & 60 & 111 & 90 & 50 & 148 & 676 \\
\hline & Expected count & 205.3 & 55 & 114.6 & 100.2 & 54 & 147 & 676 \\
\hline \multirow[t]{2}{*}{ Acceptance } & Count & 701 & 192 & 387 & 354 & 192 & 498 & 2,324 \\
\hline & Expected count & 705.6 & 189 & 394 & 344.5 & 185.5 & 505.5 & 2,324 \\
\hline \multirow[t]{2}{*}{ (Cusp of) adaptation } & Count & 58 & 11 & 49 & 35 & 21 & 60 & 234 \\
\hline & Expected count & 71 & 19 & 39.7 & 34.7 & 18.7 & 50.9 & 234 \\
\hline \multirow[t]{2}{*}{ Total } & Count & 1,012 & 271 & 565 & 494 & 266 & 725 & 3,333 \\
\hline & Expected count & 1,012 & 271 & 565 & 494 & 266 & 725 & 3,333 \\
\hline
\end{tabular}

The observed (counted) and the expected frequency of the PO for the amount of lessons about own culture or any foreign cultures the students had are similar (Table 26). 
A chi-square test of independence was performed to examine the relation between the students PO and the amount of lessons about own culture or any foreign cultures the students had. The relation between the students' PO and the amount of lessons about own culture or any foreign cultures the students had was not significant, $X^{2}(15, N=3,333)=$ $17.89, p=.27$. The null hypothesis that there is no relationship between the amount of lessons about own culture or any foreign cultures the students had and the students' stage on the IDC cannot be rejected. It can be concluded that the students' PO is independent of the amount of lessons about own culture or any foreign cultures the students had.

\section{Relationship Between the Amount of Lessons About Own Culture or Any Foreign Cultures the Students Had and Their Developmental Orientation}

The variable amount of lessons about own culture or any foreign cultures the students had has the six levels no, less than 1 day, less than 3 days, less than 1 week, less than 2 weeks, and more than 2 weeks.

Table 27 
Observed and Expected Frequency of Developmental Orientation for Amount of Lessons on Culture

\begin{tabular}{|c|c|c|c|c|c|c|c|c|}
\hline & & no & $\begin{array}{l}\text { less } \\
\text { than } 1 \\
\text { day }\end{array}$ & $\begin{array}{l}\text { less } \\
\text { than } 3 \\
\text { days }\end{array}$ & $\begin{array}{l}\text { less } \\
\text { than } 1 \\
\text { week }\end{array}$ & $\begin{array}{l}\text { less } \\
\text { than } 2 \\
\text { weeks }\end{array}$ & $\begin{array}{l}\text { more } \\
\text { than } 2 \\
\text { weeks }\end{array}$ & Total \\
\hline \multirow[t]{2}{*}{ Denial } & Count & 68 & 15 & 35 & 28 & 7 & 44 & 197 \\
\hline & Expected count & 59.8 & 16 & 33.4 & 29.2 & 15.7 & 42.9 & 197 \\
\hline \multirow[t]{2}{*}{ Cusp of polarization } & Count & 53 & 14 & 23 & 11 & 14 & 30 & 145 \\
\hline & Expected count & 44 & 11.8 & 24.6 & 21.5 & 11.6 & 31.5 & 145 \\
\hline \multirow[t]{2}{*}{ Polarization } & Count & 318 & 87 & 168 & 146 & 79 & 211 & 1,009 \\
\hline & Expected count & 306.4 & 82 & 171 & 149.5 & 80.5 & 219.5 & 1,009 \\
\hline \multirow[t]{2}{*}{ Cusp of minimization } & Count & 94 & 28 & 63 & 48 & 24 & 65 & 322 \\
\hline & Expected count & 97.8 & 26.2 & 54.6 & 47.7 & 25.7 & 70 & 322 \\
\hline \multirow[t]{2}{*}{ Minimization } & Count & 439 & 118 & 237 & 235 & 123 & 324 & 1476 \\
\hline & Expected count & 448.2 & 120 & 250.2 & 218.8 & 117.8 & 321.1 & 1476 \\
\hline \multirow[t]{2}{*}{ Cusp of acceptance } & Count & 26 & 3 & 18 & 16 & 10 & 26 & 99 \\
\hline & Expected count & 30.1 & 8 & 16.8 & 14.7 & 7.9 & 21.5 & 99 \\
\hline \multirow[t]{2}{*}{ Acceptance } & Count & 12 & 6 & 20 & 10 & 9 & 23 & 80 \\
\hline & Expected count & 24.3 & 6.5 & 13.6 & 11.9 & 6.4 & 17.4 & 80 \\
\hline \multirow[t]{2}{*}{ (Cusp of) adaptation } & Count & 2 & 0 & 1 & 0 & 0 & 2 & 5 \\
\hline & Expected count & 1.5 & 0.4 & 0.8 & 0.7 & 0.4 & 1.1 & 5 \\
\hline \multirow[t]{2}{*}{ Total } & Count & 1,012 & 271 & 565 & 494 & 266 & 725 & 3,333 \\
\hline & Expected count & 1,012 & 271 & 565 & 494 & 266 & 725 & 3,333 \\
\hline
\end{tabular}

The observed (counted) and the expected frequency of the DO for the amount of lessons about own culture or any foreign cultures the students had are similar (Table 27). A chi-square test of independence was performed to examine the relation between the students DO and the amount of lessons about own culture or any foreign cultures the students had. The relation between the students' DO and the amount of lessons about own culture or any foreign cultures the students had was not significant, $X^{2}(35, N=$ $3,333)=40.27, p=.25$. The null hypothesis that there is no relationship between the amount of lessons about own culture or any foreign cultures the students had and the students' stage on the IDC cannot be rejected. It can be concluded that the students' DO 
is independent of the amount of lessons about own culture or any foreign cultures the students had.

\section{Relationship Between the Students' Previous Education as a Health Care Assistant and Their Perceived Orientation}

The variable students' previous education as a health care assistant has the two levels: yes and no.

Table 28

Observed and Expected Frequency of Perceived Orientation for Students' Previous Education as a Health Care Assistant

\begin{tabular}{lllll}
\hline \multirow{4}{*}{ Minimization } & & Yes & No & Total \\
\cline { 2 - 5 } Cusp of acceptance & Count & 52 & 26 & 78 \\
\cline { 2 - 5 } Acceptance & Expected count & 47.7 & 30.3 & 78 \\
\cline { 2 - 5 } & Count & 371 & 217 & 588 \\
\cline { 2 - 5 } (Cusp of) adaptation & Expected count & 359.8 & 228.2 & 588 \\
\cline { 2 - 5 } & Count & 1,138 & 739 & 1,877 \\
\cline { 2 - 5 } Total & Expected count & $1,148.5$ & 728.5 & 1,877 \\
\cline { 2 - 5 } & Count & 118 & 83 & 201 \\
\cline { 2 - 5 } & Expected count & 123 & 78 & 201 \\
\cline { 2 - 5 } & Count & 1,679 & 1,065 & 2,744 \\
\cline { 2 - 5 } & Expected count & 1,679 & 1,065 & 2,744 \\
\hline
\end{tabular}

The observed (counted) and the expected frequency of the PO for the students' previous education as a health care assistant of the students were similar (Table 28). A chi-square test of independence was performed to examine the relation between the students PO and the students' previous education as health care assistant. The relation between the students' PO and the students' previous education as health care assistant was not significant, $X^{2}(3, N=2,744)=2.66, p=.45$. The null hypothesis that there is no 
relationship between the students' previous education as a health care assistant and their stage on the IDC cannot be rejected. It can be concluded that the students' PO is independent of the students' previous education as a health care assistant.

\section{Relationship Between the Students' Previous Education as a Health Care Assistant and Their Developmental Orientation}

The variable students' previous education as a health care assistant has the two levels: yes and no.

Table 29

Observed and Expected Frequency of Developmental Orientation for Students' Previous Education as a Health Care Assistant

\begin{tabular}{lllll}
\hline & & Yes & No & Total \\
\hline Denial & Count & 116 & 56 & 172 \\
\cline { 2 - 5 } Cusp of polarization & Expected count & 105.2 & 66.8 & 172 \\
\cline { 2 - 5 } Polarization & Count & 78 & 45 & 123 \\
\cline { 2 - 5 } Cusp of minimization & Exped count & 75.3 & 47.7 & 123 \\
\cline { 2 - 5 } & Count & 511 & 324 & 835 \\
\cline { 2 - 5 } Minimization & Expected count & 510.9 & 324.1 & 835 \\
\cline { 2 - 5 } & Count & 142 & 104 & 246 \\
\cline { 2 - 5 } Cusp of acceptance & Expected count & 150.5 & 95.5 & 246 \\
\cline { 2 - 5 } & Expected count & 730 & 471 & 1,201 \\
\cline { 2 - 5 } Acceptance & Count & 56 & 466.1 & 1,201 \\
\cline { 2 - 5 } (Cusp of) adaptation & Expected count & 53.8 & 34.2 & 88 \\
\cline { 2 - 5 } & Count & 45 & 30 & 75 \\
\cline { 2 - 5 } Total & Expected count & 45.9 & 29.1 & 75 \\
\cline { 2 - 5 } & Count & 1 & 1.6 & 4 \\
\hline & Expected count & 2.4 & 1,065 & 2,744 \\
\cline { 2 - 5 } & Expected count & 1,679 & 1,065 & 2,744 \\
\hline
\end{tabular}


The observed (counted) and the expected frequency of the DO for the students' previous education as a health care assistant of the students were similar (Table 29). A chi-square test of independence was performed to examine the relation between the students DO and the students' previous education as health care assistant. The relation between the students' DO and the students' previous education as health care assistant was not significant, $X^{2}(7, N=2744)=6.89, p=.44$. The null hypothesis that there is no relationship between the students' previous education as a health care assistant and their stage on the IDC cannot be rejected. It can be concluded that the students' DO is independent of their previous education as a health care assistant.

\section{Summary the Relationships Between Perceived and Developmental Orientation and Demographic Variables}

The null hypothesis that there is no relationship among the nursing students' demographic variables and their stage on the IDC can be rejected for the variables age, gender, and total amount of time having lived in another country. In conclusion, the nursing students' PO and DO is dependent on the nursing students' age, gender, and the total amount of time student lived in another country.

The null hypothesis that there is no relationship among the nursing students' demographic variables and their stage on the IDC cannot be rejected for the variables primary country of citizenship, ethnic minority group affiliation, migration background, world region having primarily lived up to age 18 , having had lessons about own culture or any foreign cultures, and previous education as a health care assistant. In conclusion, the nursing students' PO and DO is independent on the nursing students' primary country 
of citizenship, ethnic minority group affiliation, migration background, the world region students primarily lived up to age 18 , the amount of lessons about own culture or any foreign cultures the students had, and the students' previous education as a health care assistant.

\section{Results to Research Question 3}

RQ 3: To what extent does the nursing student-cohorts' orientation toward cultural difference and commonality as a group differ from the beginning to the end of their education?

Statistical procedures allow researchers to test whether the differences between two samples are large enough to conclude statistical significance. $T$ tests are used when the researcher has two sets of data such as the time-point at the start or end of the education and wishes to compare the means score on some continuous variable (Pallant, 2016). A paired samples $t$ test has been computed using SPSS. Because theoretical reasons for specifying a direction in the research hypothesis is lacking, a two-tailed test has been conducted. Although the intention of the curriculum at the college is to foster the development of the students regarding their DO toward cultural difference and commonality and the developmental movement is permanent in one direction, there may be retreats from some stages (Bennett, 2011). As the paired samples statistics demonstrate the point estimate, an estimation of the exact value of a population parameter, has its limitation. One method of increasing accuracy is to use an interval estimate rather than a point estimate. In interval estimation, "a range of values within which the population parameter may fall" is identified (Frankfort-Nachmias \& Leon- 
Guerrero, 2015, p. 239). This range of values is called a CI. Therefore, the $95 \%$ CI of the difference has been included in the paired-samples $t$ test. Alpha or the $p$-value is set at .05 , meaning that the null hypothesis was rejected if the probability of the obtained statistic is less than or equal to .05 .

\section{Difference Regarding Perceived Orientation}

The mean score for PO at the start was 119.5 and at the end it was 119.35 , with a sample of 40, a $S D$ of 1.43 (start point) and 1.48 (end point), and a std. error mean of 0.22613 (start point) and 0.23353 (end point).

Table 30

Paired Samples T Test of Perceived Orientation at Start Compared to End of Education

\begin{tabular}{|c|c|c|c|c|c|c|c|}
\hline \multicolumn{6}{|c|}{ Paired Differences } & \multirow[t]{2}{*}{$\mathrm{t} d f$} & \multirow[t]{2}{*}{ Sig. (2-tailed) } \\
\hline & Mean & $\begin{array}{l}\text { Std. } \\
\text { Deviation }\end{array}$ & $\begin{array}{l}\text { Std. } \\
\text { Error } \\
\text { Mean }\end{array}$ & $\begin{array}{l}95 \% \mathrm{C} \\
\text { Interva } \\
\text { Differe }\end{array}$ & $\begin{array}{l}\text { fidence } \\
\text { f the } \\
\text { e }\end{array}$ & & \\
\hline & & & & Lower & Upper & & \\
\hline & -0.30 & 1.41 & 0.22 & -0.75 & 0.15 & -1.3439 & .19 \\
\hline $\begin{array}{l}\text { Score of PO at } \\
\text { the start-point } \\
\text { and end-point of } \\
\text { the education }\end{array}$ & & & & & & & \\
\hline
\end{tabular}

The statistics of the $t$ test demonstrate that the mean score of PO at the end-point increased compared to the start-point of the education. However, the increase is only from 119.05 to 119.35 , that is an increase (difference between the two values) of 0.3 . Also, the $95 \%$ CI of the difference lies only between -0.75 and 0.15 (Table 30 ). The result of the paired samples t test shows a p-value of .19 (Table 30). As the threshold was set by .05 , the difference between the increase of the mean score of PO at the end-point 
compared to the start-point of the education is not statistically significant. Accordingly, the research question to what extent the nursing student-cohorts' PO toward cultural difference and commonality as a group differs from the beginning to the end of their education can be answered as follows. The nursing student-cohorts' PO toward cultural difference and commonality as a group did not significantly differ from the beginning to the end of their education.

\section{Difference Regarding Developmental Orientation}

The mean score for DO at the start was 86.33 and at the end it was 87.99 , with a sample of 40, a $S D$ of 3.83 (start point) and 4.15 (end point), and a std. error mean of 0.60496 (start point) and 0.65577 (end point).

Table 31

Paired Samples T Test of Developmental Orientation at Start Compared to End of Education

\begin{tabular}{|c|c|c|c|c|c|c|c|c|}
\hline \multicolumn{6}{|c|}{ Paired Differences } & $\mathrm{t}$ & df & Sig. (2-tailed) \\
\hline & Mean & $\begin{array}{l}\text { Std. } \\
\text { Deviation }\end{array}$ & $\begin{array}{l}\text { Std. } \\
\text { Error } \\
\text { Mean }\end{array}$ & $\begin{array}{l}95 \% \mathrm{C} \\
\text { Interva } \\
\text { Differe }\end{array}$ & $\begin{array}{l}\text { nfidence } \\
\text { of the } \\
\text { ice }\end{array}$ & & & \\
\hline & & & & Lower & Upper & & & \\
\hline $\begin{array}{l}\text { Score of DO at } \\
\text { the start-point } \\
\text { and end-point } \\
\text { of the education }\end{array}$ & -1.66 & 4.03 & 0.64 & -2.95 & -0.38 & -2.61 & 39 & .01 \\
\hline
\end{tabular}

The statistics of the $t$ test demonstrate that the mean score of DO at the end-point increased compared to the start-point of the education. The increase is from 86.33 to 87.99 , what is an increase (difference between the two values) of 1.66 . The $95 \%$ CI of the difference lies between -2.95 and -0.38 (Table 31). The result of the paired samples $t$ test 
shows a $p$-value of .01 (Table 31). As the threshold was set by .05, the difference between the increase of the mean score of DO at the end-point compared to the startpoint of the education is statistically significant. Accordingly, the research question to what extent the nursing student-cohorts' developmental orientation toward cultural difference and commonality as a group differs from the beginning to the end of their education can be answered as follows: The nursing student-cohorts' developmental orientation toward cultural difference and commonality as a group significantly differed from the beginning to the end of their education.

\section{Difference Regarding the Orientation Gap}

From a frequency sample of 40 , the orientation gap started with a mean of 32.73 and ended with a mean of 31.36, a SD of 2.46 and 2.73, and a std. error mean of 0.38953 and 0.43236 .

Table 32

Paired Samples T Test of Orientation Gap at Start Compared to End of Education

\begin{tabular}{|c|c|c|c|c|c|c|}
\hline \multicolumn{5}{|c|}{ Paired Differences } & $\mathrm{t}$ & $\begin{array}{ll} & \text { Sig. (2- } \\
\text { df } & \text { tailed) }\end{array}$ \\
\hline & Mean & $\begin{array}{l}\text { Std. } \\
\text { Deviation }\end{array}$ & $\begin{array}{l}\text { Std. } \\
\text { Error } \\
\text { Mean }\end{array}$ & $\begin{array}{l}95 \% \text { Confidence } \\
\text { Interval of the } \\
\text { Difference }\end{array}$ & & \\
\hline & & & & Lower Upper & & \\
\hline $\begin{array}{l}\text { Orientation gap at the } \\
\text { start-point and end- } \\
\text { point of the education }\end{array}$ & 1.37 & 2.73 & 0.43102 & $0.49 \quad 2.24$ & 3.17 & 39.003 \\
\hline
\end{tabular}

The statistics of the $t$ test demonstrate that the mean orientation gap at the endpoint decreased compared to the start-point of the education. The decrease is from 32.73 to 31.36 , what is a decrease (difference between the two values) of 1.37 . The $95 \%$ CI of 
the difference lies between 0.49 and 2.24 (Table 32). The result of the paired samples $t$ test shows a $p$-value of .003 (Table 32). As the threshold was set by .05 , the difference between the increase of the mean orientation gap at the end-point compared to the startpoint of the education is statistically significant. Accordingly, the research question to what extent the nursing student-cohorts' orientation gap toward cultural difference and commonality as a group differs from the beginning to the end of their education can be answered as follows. The nursing student-cohorts' orientation gap toward cultural difference and commonality as a group significantly differed from the beginning to the end of their education.

\section{Results to Research Question 4}

RQ 4: What is the relationship between the difference regarding the nursing student-cohorts' orientation toward cultural difference and commonality at the start-point and end-pont of education and the demographic variables of the cohort (years of education, year of curriculum, workload, focus of specialization, and location of school)?

Because only the nursing student-cohorts' developmental orientation toward cultural difference and commonality as a group significantly differs from the beginning to the end of their education, the analysis to test the hypotheses regarding the cohorts' orientation-difference and the demographic variables of the nursing students-cohort is limited to the developmental orientation. The data did not include more than one level of curriculum and focus of specialization. In addition, the data included one single case representing the workload part-time. Therefore, it was not possible to analyze the relationship between the cohorts' orientation-difference and the variables year of 
curriculum (2010 or 2015), workload (full-time or short-time), and focus of specialization (physically ill patients, psychiatry, pediatrics/obstetrics/gynecology).

The relationship between the interval variable cohorts' orientation-difference regarding the DO and the two categorical variable location of schools (head location in a larger city or branch location in a smaller city in a mountainous area) and years of education (three, two and a half, or two years) was analyzed by applying the eta correlation. Eta is a coefficient of nonlinear association with an interval level dependent variable and a categorical independent variable (Siegel, 1956).

With the values 0.097 for the location of school, 0.159 for the duration of the education regarding the DO, and the values 0.093 for the location of school, 0.122 for the duration of the education regarding the orientation gap, the alternate hypothesis that the cohorts' orientation-difference is different for the nursing student-cohorts according to the variables duration of the education (two, two and a half, three, or four years) or location of school lessons (head location in a larger city or branch location in a smaller city in a mountainous area) is not strongly supported. It can be concluded that there is a small if any relationship between the cohorts' orientation-difference and the nursing students' duration of the education or location of school lessons.

\section{Summary}

For RQ 1, the combination of the 2,298 nursing students being assessed at the start-point of their education with those 1,112 nursing students being assessed at the endpoint of their education provided the following results: $68 \%$ of all 3410 nursing students had a PO representing the mindset acceptance $(M=118.8, S D=5.1, \min =113.7, \max =$ 
123.9 , variance $=26.5)$ whereas $68 \%$ of them had a DO representing the mindset minimization $(M=86.3, S D=13.7, \min =72.6, \max =100$, variance $=187.9)$. In summary, the distribution of the PO and DO scores among the nursing students at the start-point and the end-point of education can be considered as reasonably normal with most scores occurring in the center, tapering out toward the extremes. However, the variation is very high particularly for the DO and overall the distribution is light-tailed and rather clustered to the left at the low values.

The results for the PO are shifted more right compared to the DO. This difference is demonstrated by a mean orientation gap between 31.3 and 33.1. A gap score of 7 points or higher can be considered a meaningful difference between where the nursing students perceive they are on the developmental continuum and where the IDI places their level of intercultural competence (IDI, 2018b). The Skewness for the orientation gap is negative with values between -0.2 and -0.3 indicating a clustering of scores at the high end and the Kurtosis value below zero indicating a distribution that is relatively flat (min $=3.7, \max =65.8)$.

For RQ 2, the null hypothesis that there is no relationship among the nursing students' demographic variables and their stage on the IDC can be rejected for the variables age, gender, and total amount of time having lived in another country. The nursing students' PO and DO is dependent on the nursing students' age, gender and the total amount of time student lived in another country. The null hypothesis that there is no relationship among the nursing students' demographic variables and their stage on the IDC cannot be rejected for the variables country of citizenship, ethnic minority group 
affiliation, migration background, world region having primarily lived up to age 18 , having had lessons about own culture or any foreign cultures, and previous education as a health care assistant. The nursing students' PO and DO is independent on the nursing students' country of citizenship, ethnic minority group affiliation, migration background, the world region students primarily lived up to age 18 , the lessons about own culture or any foreign cultures the students had, and the students' previous education as a health care assistant.

For RQ 3, the result of the paired samples $t$ test demonstrated significance for the DO $(p=.01)$ and the orientation gap $(p=.003)$, but not for the PO $(p=.187)$ regarding the difference of the nursing student-cohorts' orientation difference between the startand end-point of their education. Therefore, the nursing student-cohorts' orientation toward cultural difference and commonality as a group significantly differed from the beginning to the end of their education regarding the DO and the orientation gap but did not significantly differ regarding the PO.

For RQ 4, according to the small values of the Eta-correlation it can be concluded that there is a small if any relationship between the cohorts' orientation-difference regarding developmental orientation respectively orientation gap and the nursing students' duration of the education $(2,2.5,3$, or 4 years) or location of school lessons (head location in a larger city or branch location in a smaller city in a mountainous area). It was not possible to analyze the relationship between the cohorts' orientation-difference and the variables year of curriculum (2010 or 2015), workload (full-time or short-time), 
and focus of specialization (physically ill patients, psychiatry, pediatrics/obstetrics/ gynecology) because the data was limited.

In the next chapter the findings of this study will be compared to what has been found in the peer-reviewed literature described in Chapter 2. The focus will be on the question in what ways the findings confirm, disconfirm, or extend knowledge in nursing education. Furthermore, the findings will be analyzed and interpreted in the context of the IDI as this was the theoretical framework of this study. 
Chapter 5: Discussion, Conclusions, and Recommendations

\section{Introduction}

The purpose of this quantitative cross-sectional study with a secondary data analysis was to identify the orientation toward cultural difference and commonality on the IDC of nursing students in Switzerland and to better understand the development of intercultural competence. The descriptive analysis of the data demonstrated that $68 \%$ of

the nursing students have a PO within the range between 113.7 and $123.9(M=118.8, S D$ $=+-5.1$, mindset $=$ acceptance $)$ with a low variation $($ variance 26.5$)$. On the other hand, $68 \%$ of the nursing students have a DO score within the range between 72.6 and $100(M$ $=86.3, S D=+-13.7$, mindset $=$ minimization $)$ with a higher variation $($ variance 187.9$)$. The distribution of the scores for PO and DO of the nursing students is light-tailed and clustered to the left at the low values, though the results for the PO are shifted more right compared to the DO. This difference is demonstrated by a mean orientation gap between 31.3 and $33.1(\min =3.7, \max =65.8)$. A gap score of 7 points or higher can be considered a meaningful difference between where the nursing students perceive they are on the developmental continuum and where the IDI places their level of intercultural competence (IDI, 2018b).

Although the nursing students' PO and DO is dependent on the nursing students' age, gender, and the total amount of time student lived in another country, they are independent on the nursing students' country of citizenship, ethnic minority group affiliation, migration background, the world region students primarily lived up to age 18 , 
lessons about cultures the students had, and the students' previous education as a health care assistant.

Additionally, the results of the paired samples $t$ test demonstrated significance for the DO $(p=.01)$ and the orientation gap $(p=.003)$ but not for the $\mathrm{PO}(p=.16)$ regarding the difference of the nursing student-cohorts' orientation difference between the startand end-point of their education. Therefore, the nursing student-cohorts' orientation toward cultural difference and commonality as a group significantly differed from the start-point to the end-point of their education regarding the DO and the orientation gap but did not significantly differ regarding the PO. Further, according to the small values of the Eta-correlation, there is a small if any relationship between the cohorts' orientationdifference regarding DO orientation gap and the nursing students' duration of the education or location of lessons.

\section{Interpretation}

In this section, the findings of this study will be compared to what has been found in the peer-reviewed literature described in Chapter 2. The focus will be on what ways the findings confirm, disconfirm, or extend knowledge in nursing education.

Furthermore, the findings will be analyzed and interpreted in the context of the IDI, as this was the theoretical framework of this study.

\section{Orientation Toward Cultural Difference and Commonality of the Nursing Students}

In this study, the majority (68\%) of the 3,410 nursing students who completed the IDI either at the start-point or end-point of their education had a PO score representing the mindset acceptance $(M=118.8, R=113.7-123.9, S D=5.1$, variance $=26.5)$ and a 
DO score representing the mindset minimization $(M=86.3, R=72.6-100, S D=13.7$, variance $=187.9$ ). This finding is consistent with the findings of studies conducted on health care using the IDI, which demonstrated acceptance as the major PO and minimization as the major DO with the participating health care professionals (Altshuler et al., 2003; Boggis, 2012; Bourjolly et al., 2015; Halm \& Wilgus, 2013; Halm et al., 2012; Hamre, 2012; Harder, 2018; Huckabee \& Matkin, 2012; Kruse et al., 2014; Larson, 2011; Munoz et al., 2009; Peying et al., 2012).

The transition from ethnocentricism to ethnorelativism typically includes a phase of minimizing cultural differences, where individuals focus more on the similarities between people groups (Bennett, 1993, Hammer, 2012). The range of scores defining the mindset of minimization is between $85-115$; although every other mindset has a range of 15 points, the minimization has a range of 30 points on the scale. With 86.3 the DO mean score in this study is low, representing an early stage of minimization. Considering the high variance $(187.9)$ and the range $(\min =72.6 ; \max =100)$, a part of the students still has a mindset of polarization. However, it cannot be concluded from the result to what extent the nursing students having a mindset of minimization already changed their mindset in the direction of ethnorelativism and how strong their mindset of ethnocentrism still is. The theoretical framework only defines minimization as a transition stage between the ethnocentrism mindsets of denial and defense to the ethnorelative mindsets of acceptance and adaptation. Because the majority of health care providers being researched in the last 15 years represented a DO with this mindset, further work is 
necessary to better understand the minimization scale in relation to the transition from an ethnocentristic to an ethnorelative mindset.

To answer why health care providers score high on minimization, various aspects needs to be considered. For instance, participants having a minimization score higher on the following two individual statements in the IDI reflect an agreement in universal values, minimizing cultural differences: "Despite some cultural differences, it is more important to recognize that people are all alike in their humanity" and "People are fundamentally the same despite apparent differences in cultures” (IDI, 2018a). Additionally, cultural differences are subordinated to the similarity of peoples' biological culture and religious or other philosophical values are seen as universal absolutes (Hammer et al., 2003). The fact that physical and biological mechanisms are strongly related to disease processes may support the focus on minimizing differences among human beings (Altshuler et al., 2003). Another explanation may be that minimization may be a socially acceptable coping strategy for a health care provider to avoid the emotional stress of dealing with cultural differences along with the other challenges faced in the health care setting (Altshuler et al., 2003). However, IDI results in the general population tend to follow a bell-curve or normal distribution. Therefore, approximately $68 \%$ of the general population will fall within the minimization category, $28 \%$ will fall in the polarization and acceptance categories, and $4 \%$ at the tail ends of the denial and adaptation categories (Kruse, 2014; IDI training November 2017). The results of this study reflect trends that nursing students included in this study represent the general population. 
Similar to the finding in this study, other studies on health care using the IDI have shown high scores regarding the orientation gap and participants overestimating their level of intercultural competence. Therefore, self-perception seems not to be an adequate measure of cultural competency (Kruse et al., 2014). An orientation gap score of 7 points or higher can be considered a meaningful difference between where the participants perceive they are on the developmental continuum and where the IDI places their level of intercultural competence (IDI, 2018b). The results of this study demonstrate a mean orientation gap of 33.1 at the start-point and 31.3 at the end-point of education. Previous research has also reported a high score on the orientation gap: 22.2 for the pretest and 22.49 for the posttest (Boggis, 2012); 24.97 for the pretest and 26.26 for the posttest (Halm \& Wilgus, 2013); 23.8 for group 1 and 31.72 for group 2 (Hamre, 2012); 30.68 for the pretest and 28.96 for the posttest (Harder, 2018); 28.82 at the time of graduation (Huckabee \& Matkin, 2012); and approximately 27 (only the PO and the DO; Kruse et al., 2014).

Although these studies report an orientation gap being at least more than 3 times as high as the limit to be considered as a meaningful difference, only some of them report an orientation gap being 4 times as high, and this study showed an orientation gap even higher than four times as high. The results demonstrate that in some studies the participants' orientation gap increased from pre- to posttest, whereas other studies observed a decrease. To better understand the meaning of a meaningful orientation gap and its consequences, a more detailed examination of the differences between the participants of this study and others without such a discrepancy is recommended. 
Although the theoretical framework does not provide any further informative basis, the orientation gap identified in this study needs to be considered as meaningful, as it indicates that the nursing students overestimate their competence in this area.

Health care providers may overestimate their level of intercultural competence for a variety of reasons. According to Altshuler et al. (2003), it may be that "the participants see themselves as operating (successfully, in their view) in a challenging multicultural environment, and may have been hesitant about presenting themselves as less than competent in this regard on the IDI" (pp. 397-398). The nursing students included in this study were not informed about their IDI results; though the faculty offered to provide the result of the cohort to those interested, there was no cohort who made use of this offer. To increase the awareness of nursing students' overestimation of their current DO toward cultural difference and commonality the information about the IDI result could be valuable for self-reflection on their development of competence (Harder, 2018). As a requirement of the IDI, the information of the results need to be given within a meeting with an IDI qualified administrator (IDI, 2018a). Such a meeting may encourage nursing students to reflect on their results and consider the significance of their results in terms of further cultural development (Kruse et al. 2014). The IDI provides an individual intercultural development plan appropriate to the individual IDI result. This plan enables students to gain insights concerning intercultural challenges they are facing and identify intercultural competence development goals that are important for them, gain increased understanding of how their DO impacts how they perceive and respond to cultural difference and commonality, and identify and engage in targeted, developmental learning 
that increases their intercultural competence in bridging across diverse communities (IDI, 2018c). Students being required to self-direct their (lifelong) intercultural learning process need to get an orientation on where they stand regarding their development and where they need to go. Therefore, providing the IDI results including the required guidance to the students is essential for a constructive developmental learning process.

\section{Relationships Between the Nursing Students' Orientation and Their Demographic}

\section{Variables}

The findings of this study indicated that the nursing students' PO and DO is dependent on the nursing students' age, gender, and the total amount of time student lived in another country, but they were independent of the nursing students' country of citizenship, ethnic minority group affiliation, migration background, world region primarily lived up to age 18 , the lessons about own culture or any foreign cultures, and previous education as a health care assistant. It is essential to compare these findings with the findings of previous studies regarding the relationship between the participants' orientation toward cultural difference and commonality and the variables included in this study.

Variable gender. The finding of this study indicated that the nursing students' PO and DO is dependent on the nursing students' gender. Other studies using the IDI have reported various results. For example, Altshuler et al. (2003) found a significant relationship, as the results showed that the female residents exhibited lower denial and defense and higher acceptance and adaptation compared to their pretraining scores, and males showed an increase in denial and defense and a decrease in acceptance and 
adaptation. Conversely, Kruse et al. (2014) did not find statistically significant differences between undergraduate and graduate students or differences related to gender.

Other studies with different theoretical frameworks have demonstrated various results. Riley, Smyer, and York (2012) found no significant relationships between gender and total scores or any of the construct scores $(p=.05)$. De Beer and Chipps (2014) also did not find significant differences in cultural competence for gender. However, Baghdadi and Ismaile (2018) demonstrated by using regression analysis that demographic variables such as gender were predictive of the mean score. Similarly, Cruz et al. (2018) identified gender as a factor affecting cultural competence.

Variable age. The findings of this study indicate that the nursing students' PO and DO is dependent on the nursing students' age, which other studies using the IDI have similarly reported. Kruse et al. (2014) found a statistically significant difference in PO scores for the five age groups (18-21, 22-30, 31-40, 41-50, and 51-61 years): $F(4,260)$ $=2.48, p=.04)$. Similar to this study, Kruse et al. reported that despite the statistical significance, the actual difference in mean scores between the groups was small, as evidenced by an eta-squared value of .04. However, Kruse et al. found no statistically significant difference in scores for the five age groups $(F(4,260)=2.28, p=.06)$ in terms of developmental orientation and age. Hamre (2012) examined the PO, the DO, and the orientation gap using ANOVA, but no significant relationships were present $(p<.05)$. ANOVA was undertaken to look for correlation in the initial IDI results between age and the clusters and subclusters, which again revealed no significant findings, although the adaptation cluster: cognitive frame-shifting was trending toward significance $(F=3.229$, 
$d f 1=2, d f 2=20, p=.06)$. Hamre concluded that the findings were slightly different for one of the two groups because only one participant in the total sample was in the 22 to 30 category and the rest of the participants were in the 18 to 21-category. Looking at the results of age, it must be considered that the range of years in the age categories varies. A higher range of years may result in less understanding of participants' actual age compared to a range of fewer years (Hamre, 2012).

Other studies with different theoretical frameworks have demonstrated various results. Riley et al. (2012) found significant differences between total score and age, as well as a higher total, mean score (75.3) compared with what Diaz et al. (2015) reported in their study (71.6). ANOVA tests have identified a significant difference in cultural skill construct scores, with the youngest respondents, 20-30, recording the highest mean scores. However, De Beer and Chipps (2014) did not find significant differences in cultural competence for the variable age. Additionally, Diaz et al. (2015) found that applying multiple linear regression age could not be used as variable to predict cultural competence levels of nurse educators, clinical educators, or graduate students. Finally, Baghdadi and Ismaile (2018) demonstrated that demographic variables such as age were predictive of the mean score, and Cruz et al. (2018) identified age as a factor affecting cultural competence.

Variables primary country of citizenship, ethnic minority group affiliation, migration background, world region primarily lived up to age 18, and amount of time lived in another country. The findings of this study indicated that the nursing students' PO and DO is dependent on the nursing students' total amount of time lived in 
another country, but they are independent of the nursing students' primary country of citizenship, ethnic minority group affiliation, and migration background. These findings are inconsistent with the findings of Kruse et al. (2014), who conducted a one-way ANOVA to explore the differences in PO and DO scores among time-abroad categories (never, $<1$ month-11months, $1-5$ years, 6-10 years, and $>10$ years). Both PO scores $F$ $(4,270)=2.06, p=.09$ and DO scores $F(4,270)=1.42, p=.23$ were not statistically significant for differences between groups. Kruse et al. also found statistically significant differences in PO and DO scores for ethnic minorities compared to ethnic nonminorities. Minority students had higher DO scores $(M=98.85, S D=14.21)$ than nonminority students $(M=94.4, S D=14.96)$. These results did not support that IDI results may be generalizable across cultural groups with results not differing between ethnic minorities and nonminorities (Hammer, 2009). Kruse et al. considered that the reason for the statistically significant higher scores for minority students is because these students are continuously exposed to and navigate in cultures of others. Additionally, Hamre (2012) examined the relation between the DO and the world region students primarily lived up to age 18 , not finding a significant relationship $X^{2}(1, N=29)=1.168, p=.28$. Hamre explained that in one group all participants spent their formative years in North America, whereas in the other group only two participants spent their formative years in Africa.

Other studies with different theoretical frameworks have demonstrated various results. Riley et al. (2012) did not identify any significant relationships between race and IAPCC-R scores. De Beer and Chipps (2014) found out that nurses from non-Englishspeaking backgrounds scored significantly higher in cultural competence than English- 
speaking nurses. Analyzing the data by English-speaking background, the higher cultural competence score for non-English-speaking nurses (primarily Zulu-speaking) was driven by significant differences in cultural encounters $(p<.001)$, cultural desire $(p=.02)$ and cultural awareness $(p=.02)$. Furthermore, significant differences were found for the race $(p<.001)$, with lack nurses scoring significantly higher compared with the other races.

Regarding the amount of time having lived abroad, it is essential to consider that a greater number of years abroad does not necessarily mean an increase in intercultural competence. For example, someone who has spent a longer time in other countries without making it a meaningful educational journey may still have an ethnocentristic mindset, whereas someone having experienced guided cultural immersion activities in his or her home country could have a more ethnorelative mindset (Kruse et al., 2014).

Variable previous education as a health care assistant. The findings of this study indicating that the nursing students' PO and DO is independent on the nursing students' previous education as health care assistant cannot be compared to another study within health care using the IDI, as none has been published so far. As the education as health care assistant is unique to Switzerland and no other study within health care has been published so far, the findings of studies examining relationships to variables regarding the experience with working in nursing or with diversity within patients or team members at workplace will be highlighted. Hamre (2012) performed a chi-square test of independence to examine the relation between the DO and the variables experiences and age. The relationship for these variables was not significant for the total sample $X^{2}(6, N=29)=4.035, p=.67$ nor the analytic sample $X^{2}(4, N=9)=1.386, p=$ 
.85 regarding the. She informed that the age category increased with the experience category, except of the oldest participant having no experience and explained the lack of significance between age and experience as "surprising and likely a result of the small sample size" (p. 69).

The other studies reporting about these variables used different theoretical frameworks and demonstrated various results. Cicolini et al. (2015) who investigated in Italy within 1,432 nurses by using the Cultural Competence Assessment tool (Doorenbos, Schim, Benkert, and Borse, 2005) found out that educational level and years of clinical experience were significantly associated with the measured cultural competence level. Riley et al. (2012) found moderate, negative correlations between years of experience and both the cultural knowledge construct $(r=-.342, p=.05)$ and the cultural skill construct $(r=-.347, p=.05)$. A weak, negative correlation existed between years of nursing experience and total IAPCC-K scores $(r=-.294, p=.05)$. Alpers and Hanssen (2014) who investigated within nurses working in a medical unit and psychiatric unit at a hospital in Norway with a 30-40\% ethnic minority patient population found out that there were no significant differences between the two groups and neither the years of work experience, not the respondents' ages did affect the responses. Corresponding with the findings, the authors concluded that "experience alone does not equip nurses with adequate knowledge for intercultural symptom assessment and culturally competent treatment and care" (p. 1003). The nurses need to be guided by reflection on their particular context to learn from experienced situations to become more interculturally competent. 
Mareno and Hart (2014) who compared the level of cultural awareness, knowledge, skills, and comfort of 365 nurses with undergraduate and graduate degrees when encountering patients from diverse populations in the Southeastern States (USA) by using the IAPCC-R by Campinha-Bacote (2018b) found that undergraduate-degree nurses scored lower than graduate-degree nurses on cultural knowledge. Scores on cultural awareness, skills, and comfort with patient encounters did not vary between groups. Cruz, Estacio, Bagtang, and Colet (2016) who investigated within 332 nursing students in the Philippines by using the Cultural Capacity Scale (Perng \& Watson, 2012) identified the variables living in an environment with culturally diverse people, prior diversity training, being in the latter years of the nursing program, and with experience of caring for patients from diverse cultures and special population groups, as predictors, accounting for $68.1 \%$ of the variance of cultural competence. Most recently, Cruz et al. (2018) who investigated within 2,163 nursing students from nine countries using the Cultural Capacity Scale (Perng \& Watson, 2012) identified the experience of taking care of patients from culturally diverse backgrounds and patients belonging to special population groups and living in a multicultural environment as factors affecting cultural competence. Chan and Nyback (2015) conducted a qualitative study to explore and describe how a virtual course in intercultural communication between 10 Junior year nursing students of Hongkong in China and ten students of Finland, through internationalization at home, can support the development of cultural competence. Chan and Nyback concluded that the students learned to appreciate each other as people with cultural differences yet with much in common and to recognize their assumptions and the 
value of subtleties and nuances through experiential intercultural encounters without leaving home.

Variable lessons about culture. The findings of this study indicating that the nursing students' PO and DO is independent on the amount of lessons about own culture or any foreign cultures the nursing students had cannot be compared to another study within health care using the IDI, as other studies did not include such a variable distinguished from the interventions included in the studies. One other study reporting about this variable used a different theoretical framework and demonstrated different results.

Cicolini et al. (2015) found that more than $50 \%$ of the researched 1,432 nurses in Italy had participated in some kind of cultural diversity training. The findings showed a significant association between the nurses' culturally competent behavior scores and prior diversity training $(p<.001)$. Participation in more than one training event was also associated with higher culturally competent behavior scores $(p<.001)$.

\section{Foster the Development of the Orientation Toward Cultural Difference and Commonality of the Nursing Students}

The result of the paired samples t test demonstrated significance for the DO $(p=.01)$ and the orientation gap $(p=.003)$, but not for the PO $(p=.19)$ regarding the difference of the nursing student-cohorts' orientation difference between the start- and end-point of their education. Therefore, the nursing student-cohorts' orientation toward cultural difference and commonality as a group significantly differed from the beginning to the end of their education regarding the DO and the orientation gap but did not 
significantly differ regarding the PO. No other study within health care using the IDI reported significant results regarding the increase of the participants DO between time one (pretest) and time two (posttest).

Altshuler et al. (2003) reported that pre- and post-training IDI scores were analyzed using the three-factor model and no significant differences were found for either of the two training groups. Boggis (2012) presented that group with the occupational therapy students did not show a significant change in overall DO mean scores from pretest to posttest $(t=0.847, p=0.41)$ while the control group showed a significant decrease in developmental orientation mean scores at posttest $(t=6.1, p<.001)$. Halm and Wilgus (2013) found out that the scores did not change significantly from pretest $(M=97.2)$ to posttest $(M=95.91)$. Halm et al. (2012) conducted the $t$ tests, which showed no significant differences between pre- and the posttest scores $(p>.05)$. Hamre (2012) identified that both included groups experienced higher scores on their PO as well as their DO, but by using $t$ tests neither the difference for the PO $(p=.88)$ nor the DO $(p=.94)$ was statistically different. Harder (2018) observed a 3.38 gain in score while both the score at the pre- and at posttest place the groups' development orientation within the mindset of minimization without reporting any significance level. Peying et al. (2012) reported that the DO score increased from pretest $(M=92.91)$ to posttest $(M=97.22)$ but did not reach statistical significance $(p=.10)$.

The comparison between the significant result of this study and the nonsignificant results of the other studies needs to be considered carefully because the studies vary regarding intervention, duration, design, and scope. Most researchers 
developed their own training program with various content and design including duration. The researchers applied the following interventions to foster the intercultural competence of the participants: (a) various educational training programs in a classroom (Altshuler et al., 2003; Bourjolly et al., 2005: Halm \& Wilgus, 2013) and experimental learning such as (b) in-service session on the end-of-life care (Halm et al., 2012), (c) video and simulation (Harder, 2018), and d) 4-week international placement (Peying et al., 2012). Huckabee and Matkin (2012) combined the two approaches by applying didactic instruction, learning activities, group projects, and supervised patient care at a free clinic for the homeless. Similarly, Altshuler et al. (2003) applied a workshop and a culture OSCE (objective structured controlled evaluation). Most researchers used reflective learning methods such as journal-writing and analysis of experiences, those researchers using a mixed method design retrieved the qualitative data from those writings. Some researchers did not apply any interventions as the study design was descriptive (Hamre, 2012; Kruse et al., 2014; Larson, 2011). Because the time between the pre- and the posttest was different according to the various duration of the interventions the duration of the studies was heterogeneous. Halm et al. (2012), Halm and Wilgus (2013), Harder (2018), and Peying et. al (2012) assessed the participants' orientation toward difference and commonality twice within a short time period of some weeks while Huckabee and Matkin (2012) investigated over a five-year period and Boggis (2012) over a three-year period. Although Munoz et al. (2009) also applied an intervention, they assessed the intercultural competence only once more or less at the beginning of the intervention. 
Therefore, the results of this and the other studies within health care using the IDI may be considered carefully as the heterogeneity needs to be seen as a limitation.

The fact that this study identified a significant increase of the DO $(p=.012)$ from the start-point to the end-point of the education might be related to the consequently integrated approach at the nursing college. At the nursing college in Switzerland, the development of the intercultural competence is integrated into the curriculum within the school during the entire education. This program includes that every nursing student completes a student exchange program in the last year of their higher education to the nursing diploma. An accurate preparation before the exchange and reflection during and after the exchange is emphasized to increase their intercultural competence. Furthermore, particular training sessions are integrated into the curriculum throughout the education at the college. With the intention to measure the students' orientation toward cultural difference and commonalities the IDI is mandatorily applied for every student at the college as well at the start and the end of their education. As this study did not include an experimental design, there are no statistical results regarding the relationship between this significant increase of the DO and any interventions. With regard to the available data, the relationship between the nursing student-cohorts' orientation difference between the start-point and the end-point of their education and identified demographic variables were examined. According to the small values of the Eta-correlation it can be concluded that there is a small if any relationship between the cohorts' orientation-difference regarding developmental orientation respectively orientation gap and the nursing students' duration 
of the education (two, two and a half, three, or four years) or location of school lessons (head location in a larger city or branch location in a smaller city in a mountainous area).

\section{Limitations of the Study}

The scope of this study included a particular sample (2,298 nursing students who were enrolled at the Nursing College between September 2010 and September 2016) of the entire population of nursing students in Switzerland. The numbers of students completing their nursing education at a college of higher education in Switzerland are presented in Table 33.

Table 33

Number of Students Who Started or Completed Nursing Education

\begin{tabular}{ll}
\hline Year & Number of students having started their education \\
\hline 2010 & 1418 \\
2011 & 1634 \\
2012 & 1585 \\
1213 & 1717 \\
1214 & 1548 \\
\hline Year & Number of students having completed their education \\
\hline 2010 & 1676 \\
2011 & 1340 \\
2012 & 1109 \\
1213 & 1447 \\
1214 & 1710 \\
\hline
\end{tabular}

Note. From “Ausbildung: Eintritte und Abschlüsse im Bereich Pflege, 2010-2014,” by

Obsan (2016). Gesundheitspersonal in der Schweiz - Bestandesaufnahme und Prognosen bis 2030 , p. 53 .

In 2015 there were a total of 1,713 and in 2016 total of 1,587 students who completed the education as registered nurse (called dipl. Pflegefachfrau HF/ dipl. Pflegefachmann HF) at the colleges for higher education in nursing in Switzerland (State 
Secretariat for Education Research and Innovation, 2018). With about 400 students to become registered nurses every year the Nursing College where the nursing students included in this study completed their education is the largest college of higher education in nursing in Switzerland. Therefore, the findings of this study may be generalized to other nursing students who complete their education at other colleges of higher education in nursing in Switzerland. However, other colleges have not implemented the development of the intercultural competence as extensively into their curriculum like the Nursing College. Particularly the compulsory student exchange program in the last year of education implemented at the Nursing College since 2010 is unique in Switzerland. However, applying the post facto design investigating into data already collected the sample cannot be considered random (Simon \& Goes, 2013). Therefore, a generalization of the findings needs careful consideration.

The scope of this study the study was based on the Intercultural Developmental Continuum (Hammer, 2012) and the data of nursing students assessed by using the IDI as the measurement instrument (IDI, 2018a, 2018b). Therefore, the perspective on the topic was shaped according to the propositions this theoretical framework is based on. As the development of the intercultural competence is complex, other propositions of other theories such as Campinha-Bacote's (2018) Process of Cultural Competence in the Delivery of Healthcare Services may have been neglected within this study without good reason. This fact indicates a limitation regarding the validity of this study. Qualitative data would further contribute to the knowledge by giving more insights about what contributes to or prevents the development of intercultural competence particularly in 
relation to the quantitative data. However, this study was limited to the quantitative data because of restricted resources.

The students completed the IDI within a regular school lesson. At the start-point of their education, they might not have understood very well the term culture although it is explained within the IDI. At the end of their education, the concentration to complete the IDI may have been threatened because the students came to say goodbye to each other for this very last day to school after having completed the last 6-month-internship. As well at the start- as at the end-point, the students were aware that the intention of applying the IDI was to know how their orientation toward cultural difference and commonality is. The students may have altered their responses according to what they think we would consider as a good response (Hawthorn Effect). The students may have changed their responses being evaluated only because it is being studied, and not because of the intervention (McCambridge, Kyprib, \& Elbourne, 2014). This may have happened although the study has not been designed as an experimental study, the students were aware that the purpose of taking the IDI was to evaluate the specialized program to foster their intercultural competence. The circumstances of the data collection need to be considered as a limitation of this study regarding validity and reliability.

As further limitation regarding the validity of this study needs to be considered that there might be other variables confounding the findings of this study. There was no random assignment as every nursing students at the Nursing College between 2010 and 2016 who was present in the lesson where the IDI has been conducted was included in the study. Consequently, there could be inherent confounds in the variables studied 
because of no random assignment, but also the fact that the development of the intercultural competence is dependent on other variables occurring in the students' personal characteristics or their environment as those being included in this study as independent variables.

\section{Recommendations}

Compared to other studies in health care using the IDI the sample included in the study was high with 2,298 nursing students who completed the IDI at the start-point and 1,112 nursing students who completed it at the end-point of their education. The results regarding the nursing students' orientation toward cultural differences and commonalities are similar as those of other studies, reporting that most of the participants demonstrated a PO representing the mindset acceptance and a DO representing the mindset minimization. The developmental transition from ethnocentricism to ethnorelativism typically includes a phase of minimizing cultural differences, where individuals focus more on the similarities between people groups (Bennett, 1993, Hammer, 2012). Individuals with this mindset tend to highlight commonalities across cultures, an orientation that can mask important cultural difference (Halm et al., 2012). The minimization of cultural differences may avoid cultural clashes but creates a barrier to exploring unique distinctions between cultures (Huckabee and Matkin, 2012). Opportunities to distinguish unique attributes of different cultures as well as developing frameworks for understanding their own individual cultures may help offset the tendency toward minimization (Bennett, 1993). The authors of the selected studies agree that moving professionals forward on the continuum of increased cultural competency 
remains the goal, going beyond minimization. Further studies should explore in more detail what contributes to the development of individuals with a mindset of minimization.

The difference between where the nursing students perceived they are on the developmental continuum (PO) and where the IDI placed their level of intercultural competence (DO) was higher than identified in other studies. Such a high orientation gap may influence the students' level of participation in interventions because they think they were doing much better than they really were (Boggis, 2012). The students might feel a limited challenge resulting in no progression to an ethnorelative mindset. On the other hand, if the students are challenged beyond their readiness, they might not move forward on the IDC or even retreat to a prior stage. Furthermore, students who are not appropriately prepared for cultural encounters may reinforce their negative stereotypes instead of challenging them. Although, a wide range of developmental orientations within the students, it is essential that educators provide training programs with just the right challenge that meets the students' level of readiness for cultural development. In this regard, further examinations of what challenges students how are indicated.

Reflection was a core component of the training sessions at the Nursing College to foster the development of the intercultural competence in the nursing students who were included in this study. Also, the authors of other studies consistently emphasized the importance of reflection. There is a common understanding that reflecting upon people's clinical cultural encounters and experiences is a critical component in order to make meaning of the experience. However, as reflection has not been clearly and comprehensively described it remains unclear what exactly is meant with interventions to 
bring students to reflect their experiences. Therefore, interventions such as journalwriting or analysis of experiences within peer-discussion are heterogeneous as well. Interventions such as reflection were not examined in this study because it did not include an experimental design. Further studies to explore reflection in relation to the intercultural development are recommended.

The same needs to be stated for the duration of the interventions and the time being devoted to the participants' development of intercultural competence. While this study examined the difference of the nursing students' orientation over a time period of two to four years (e.g., Huckabee and Matkin, 2012 or Boggis, 2012), some other researchers allowed a short period of some weeks (e.g., Harder, 2018 or Peying et al., 2012). The question about how sustainable a certain development is could not be answered with this study. Researchers debate whether a current intervention may rise the participants' cultural awareness only short term (Halm \& Wilgus, 2013). Therefore, it remains unclear what is needed for an individual to develop further along the IDC having a lasting effect. For the case of the nursing students being included in this study, it might be useful to assess their intercultural competence at a later time point, for instance, two years after completition of their nursing education.

Although this study may contribute to the knowledge what contributes to the development of the intercultural competence of nurses or other health care providers, the knowledge is still limited and further research essential. 


\section{Implications}

Nurses need to be prepared to care for an aging, chronically ill and diverse population in the health care setting while diversity is inherent in health care providers themselves. With the increasing diversity in the Swiss health care system in relation to the changes in the population demographic, the intercultural competence is a requirement for nurses. According to the International Council of Nursing (2013) "nurses should be culturally and linguistically competent to understand and respond effectively to the cultural and linguistic needs of clients, families, and communities in a health care encounter" (p. 1).

Nurses with a higher level of intercultural competence have more likely an orientation toward cultural difference and commonality in an ethnorelative stage on the IDC. Nurses with an ethnorelative orientation are able to deal more effectively with cultural diversity as those nurses with an orientation in an ethnocentric stage on the IDC (Hammer, 2012). An increase of the intercultural competence in nurses contributes to positive social change as nurses are providing health care service for the society and perform better with higher competencies.

This study may affect positive social change by providing more knowledge to understand the contributors to an increase in intercultural competence. Consequently, nursing educators may use this knowledge to better understand the contributors to the development of intercultural competence of nursing students. Therefore, this study may primarily affect positive social change at the individual level of the nursing students. 
However, nurses being more interculturally competent may affect positive social change on family, organizational, and societal/policy.

Positive social change may occur in the families of the nurses themselves, but also because of nurses' work in home care. The nurses dealing more effectively with diversity may be a role model for care givers of family members. Either in their professional role but also as family members and citizen, their ethnorelative orientation toward cultural difference and commonality may affect others regarding their orientation and contribute to a better understanding within families and communities. Accordingly, an increasing number of nurses being interculturally competent may affect others on a societal level. Nurses becoming politically active or serving within management may contribute to a positive social change on an organizational level and may contribute to adequate policies. Such a process is consistent with Walden University's core commitment.

Walden University's (2018) core commitment to ensure that Walden's students “can apply new skills, expand their networks, gain deeper knowledge, and consider a variety of perspectives in order to better address practical problems at an individual level as well as within their organizations, communities, and society at large" (Scholarly Change, para.1) clearly shows that the social change happens at first within the students in educating and enabling them to grow on their skills and knowledge getting more perspectives. Secondly, these students become professionals being able to address practical problems at an individual level as well as within their organizations, communities, and society at large. With this commitment, it is not stated what needs to be changed how but it directs the focus on actively addressing problems. Therefore, it is 
essential always to consider what is a problem for whom and what really needs to be socially changed why or in other words what is positive for whom.

The findings in this study indicated that a majority of the nursing students in Switzerland have an orientation toward cultural difference and commonalities minimizing cultural differences and taking true that there are universal values important for every human being (IDI, 2018a). With such an orientation, students who will become a nurse would say "I treat everyone the same." or even "I care for everybody just the way I would like to be cared for." Such an orientation is still ethnocentristic taking one's own culture as central to reality. The nursing students consider sameness as fair because they assume that the others have the same values and norms like them. The same happens when policies are made on the base of such assumptions.

Fortunately, Switzerland's unique political system is today the world's most stable democratic system, offering a maximum of participation to citizens (Direct Democracy, 2005). The very basics are decentralization of power and the unique instruments of direct democracy, what are frequent referendums and popular initiatives. Therefore, living in a society with a direct democratic system in Switzerland provides a possibility to relate in some ways to democratical decisions about what most of the society's people want. Every citizen of Switzerland can directly initiate social change in having influence. There is an extraordinary amount of participation in the political process that is granted to ordinary citizens. In other words, it is not the mere existence of direct democratic instruments (federalism is widespread, and referendums are not completely unknown to other democratic systems) but rather the frequent use of them, 
not only as encouraged by Switzerland's Constitution, but as practiced with enthusiasm by the citizens. Frequent referendums do have a stabilizing influence on parliament, government, economy, and society. However, social change occurs not only on the level of the constitution respectively political decisions; it occurs within communities respectively within the interaction of individuals.

Working as faculty at a Nursing College I am part of social change. Within a team of teachers, I educate nursing students in order to provide enough nurses needed by society. Improving the quality of our education regarding the outcome, respectively the competence of the nurses is essential, knowing that the competence of a nurse needs to relate to the need of the patients in the Swiss health care system. As those patients have diverse backgrounds, their needs cannot be defined generally. Therefore, it comes back to having a social system providing space for diversity and providing care for a diverse population according to the needs of the individuals and to the importance that educators know how they can contribute to the nursing students' development of their intercultural competence achieving a more ethnorelative mindset taking one's own and other cultures as relative to context.

\section{Conclusion}

The findings of this study indicated consistently to other studies that the nursing students' orientation toward cultural differences and commonalities represents a mindset of acceptance for the PO and a mindset of minimization for the DO. However, the difference between where the nursing students perceived they are on the developmental continuum (PO) and where the IDI placed their level of intercultural competence (DO) 
was higher than identified in other studies. This finding needs to be considered in the further development of the program at the Nursing College with regards to the students' satisfaction scores with the education. Such a high orientation gap may not only threaten the students' perception of the effectiveness of the program but also their benefit, because the students may think they were doing much better than they really were. The students might not feel challenged or may be challenged beyond their readiness. Instead of moving forward on the IDC there is a risk to retreat to a prior stage. Although, a wide range of developmental orientations within the students, it is essential that educators provide training programs with just the right challenge that meets the students' level of readiness for cultural development. Therefore, it is recommended to further develop the program at the Nursing College with regard to the following two aspects: First, the students should become aware of their orientation toward cultural difference and commonality and therefore become informed about their results of the IDI. Second, this information should be the base for further interventions with a clear focus on what particular nursing students need why. This includes decisions regarding immersion interventions such as the student's exchange program. This decision should depend on the particular nursing students' DO in order to prevent a stagnancy in the development or retreats to prior developmental stages because students were not enough or too much challenged.

This study was the first investigating within nursing students in Switzerland using the IDI. Compared to other studies within health care using the IDI the sample included in the study was high with 2,298 nursing students who completed the IDI at the start- 
point and 1,112 nursing students who completed it at the end-point of their education. In relation to the total numbers of students who completed their nursing education at the colleges of higher education in Switzerland, the results of this study may be generalized to other nursing students. However, other colleges have not implemented the development of the intercultural competence as extensively into their curriculum as the Nursing College did. Particularly the compulsory student exchange program in the last year of education implemented at the Nursing College since 2010 is unique in Switzerland. Therefore, the implementation of the intercultural competence as a central element within the curriculum at the other colleges of higher education in nursing in Switzerland following the example of the Nursing College included in this study is strongly recommended. 


\section{References}

All About Switzerland (2016). Switzerland's four national languages. Retrieved from http://official-swiss-national-languages.all-about-switzerland.info/

Albarran, J., Rosser, E., Bach, S., Uhrenfeldt, L., Lundberg, P., \& Law, K. (2011). Exploring the development of a cultural care framework for European caring science. International Journal of Qualitative Studies on Health and Well-being, 6(4),11457. http://dx.doi.Org/10.3402/qhw.v6i4.11457

Allen, J., Brown, L. Duff, C. Nesbitt, P., \& Hepner, A. (2013). Development and evaluation of a teaching and learning approach in cross-cultural care and antidiscrimination in university nursing students, Nurse Education Today, 33(12), 1592-1598. https://doi.org/10.1016/j.nedt.2012.12.006

Alpers, L. M., \& Hanssen, I. (2014). Caring for ethnic minority patients: A mixed method study of nurses' self-assessment of cultural competency. Nurse Education Today, 34(6), 999-1004. https://doi.org/10.1016/j.nedt.2013.12.004

Altshuler, L., Sussmann, N. M., \& Kachur, E. (2003). Assessing changes in intercultural sensitivity among physician trainees using the intercultural development inventory. International Journal of Intercultural Relations, 27, 387-401. https://doi.org/10.1016/s0147-1767(03)00029-4

Amerson, R. (2012). The influence of international service-learning on transcultural selfefficacy. International Journal of Teaching and Learning in Higher Education, 24(1), 6-15. https://files.eric.ed.gov/fulltext/EJ977178.pdf

Andrews, M. M., \& Boyle, J. S. (2002). Transcultural concepts in nursing care. Journal 
of Transcultural Nursing, 13(3), 178-180.

https://doi.org/10.1177/10459602013003002

Bachmann, M. (2013). Interprofessionelles Lernen: Teamwork am Spitalbett. Retrieved from http://bachmannkommunikation.ch/fileadmin/_migrated/content_uploads/Teamwork_am_Spitalbe tt.pdf

Baghdadi, N. A., \& Ismaile, S. (2018). Cultural competency of nursing faculty teaching in baccalaureate nursing programs in the United States. Australasian Medical Journal, 11(2), 126-134. https://doi.org/10.21767/amj.2018.3335

Banerjee, A., Chitnis, U. B. Jadhav, S. L. Bhawalkar, J. S., \& Chaudhury, S. (2009). Hypothesis testing, type I and type II errors. Industrial Psychiatry Journal, 18(2), 127-131. https://doi.org/10.4103/0972-6748.62274

Bennett, M. J. (1986). A developmental approach to training for intercultural sensitivity. International Journal of Intercultural Relations, 10(2), 179-195. https://doi.org/10.1016/0147-1767(86)90005-2

Bennett, M. J. (1993). Toward ethnorelativism: A Developmental Model of Intercultural Sensitivity. In M. Paige (Ed.), Education for the intercultural experience (pp. 109-135). Yarmouth, ME: Intercultural Press.

Bennett, M. J. (Ed.). (1998). Intercultural communication: A current perspective. In M. J. Bennett (Ed.), Basic concepts of intercultural communication: Selected readings (pp. 1-34). Boston, MA: Intercultural Press.

Bennett, M. J. (2001). Intercultural competence for global leadership. Retrieved from 
https://www.idrinstitute.org/wp-content/uploads/2018/02/Global_ICC_IDRI.pdf

Bennett, M. J. (2004). Becoming interculturally competent. In J. Wurzel (Ed.), Toward multiculturalism: A reader in multicultural education (2nd ed., pp. 62-77). Retrieved from https://www.idrinstitute.org/wpcontent/uploads/2018/02/becoming_ic_competent.pdf

Bennett, M. J. (2009). Defining, measuring, and facilitating intercultural learning: A conceptual introduction to the intercultural education double supplement. Intercultural Education, 20(sup1), 1-13. Retrieved from https://www.idrinstitute.org/wpcontent/uploads/2018/02/defining_measuring_facilitating.pdf

Bennett, M. J. (2011). Developing intercultural sensitivity. Retrieved from http://www.idrinstitute.org/allegati/IDRI_t_Pubblicazioni/47/FILE_Documento_ Bennett_DMIS_12pp_quotes_rev_2011.pdf

Bennett, M. J. (2013). Basic concepts of intercultural communication: Paradigms, principles, \& practices. Boston, MA: Intercultural Press.

Bleich, M. R., MacWilliams, B. R., \& Schmidt, B. J. (2015). Advancing diversity through inclusive excellence in nursing education. Journal of Professional Nursing, 31(2), 89-94. https://doi.org/10.1016/j.profnurs.2014.09.003

Boggis, D. T. (2012). Effectiveness of a developmental curricular design to graduate culturally competent health practitioners. Journal of Allied Health, 41(3), 140146. Retrieved from https://www.proquest.com/

Bourjolly, J. N., Sands, R. G., Solomon, P., Stanhope, V., Pernell-Arnold, A., \& Finley, 
L. (2005). The journey toward intercultural sensitivity. Journal of Ethnic and Cultural Diversity in Social Work, 14(3-4), 41-62.

http://dx.doi.org/10.1300/J051v14n03_03

Brown, B., Warren, N. S., Brehm, B., Breen, P., Bierschbach, J. L., Smith, R., \& Van Loon, R.A. (2008). The design and evaluation of an interprofessional elective course with a cultural competence component. Journal of Allied Health, 37(4), 316E-337E. https://doi.org/10.7710/2159-1253.1103

Cai, D. Y. (2016). A concept analysis of cultural competence. International Journal of Nursing Sciences, 3, 268-279. https://doi.org/10.1016/j.ijnss.2016.08.002

Campinha-Bacote, J. (2002). The process of cultural competence in the delivery of health care services: A model of care. Journal of Transcultural Nursing, 13(3), 181-184. https://doi.org/10.1177/10459602013003003

Campinha-Bacote, J. (2018a). The process of cultural competence in the delivery of health care services. Retrieved from http://transculturalcare.net/the-process-ofcultural-competence-in-the-delivery-of-healthcare-services

Campinha-Bacote, J. (2018b). Inventory for assessing the process of cultural competence among healthcare professionals- revised (IAPCC-R). Retrieved from http://transculturalcare.net/iapcc-r/

Campinha-Bacote, J. (2018c). Inventory for assessing the process of cultural competence among healthcare professionals- student version (IAPCC-SV). Retrieved from http://transculturalcare.net/iapcc-sv/

Cattacin, S., Gerber, B., Sardi, M., \& Wegener, R. (2006). Monitoring rightwing 
extremist attitudes, xenophobia and misanthropy in Switzerland (Research report - PNR 40+). Geneva: University of Geneva. Retrieved from https://www.unige.ch/sciencessociete/socio/files/6614/0533/5966/WEB_SOCIOGRAPH_1_complet.pdf Cerezo, P. G., Galceran, M. S., Soriano, M. G., Camps, L. M., \& Moral, J. M. L. (2014). Design and evaluation of an educational course in cultural competence for nursing. Procedia - Social and Behavioral Sciences, 132, 262-268. https://doi.org/10.1016/j.sbspro.2014.04.308

Chan, E. A., \& Nyback, M. H. (2015). A virtual caravan - A metaphor for homeinternationalization through social media: A qualitative content analysis. Nurse Education Today, 35(6), 828-832. https://doi.org/10.1016/j.nedt.2015.01.024

Cicolini, G., Della Pelle, C., Comparcini, D., Tomietto, M. Cerratti, F., Schim, S. M., .. . Simonetti, P. (2015). Cultural competence among Italian nurses: A multicentric survey. Journal of Nursing Scholarship, 47(6), 536-543. https://doi.org/10.1111/jnu.12165

Cowan, D. T., \& Norman, I. (2006) Cultural competence in nursing: New meanings. Journal of Transcultural Nursing, 17(1), 82-88. https://doi.org/10.1177/1043659605281976

Cruz, J. P., Estacio, J. C., Bagtang, C. E., \& Colet, P. C. (2016). Predictors of cultural competence among nursing students in the Philippines: A cross-sectional study. Nurse Education Today, 46, 121-126. https://doi.org/10.1016/j.nedt.2016.09.001 Cruz, J. P., Aguinaldo, A. N., Estacio, J. C., Alotaibi, A., Arguvanli, S., Cayaban, A. R., . 
.. Sebaeng, J. (2018). A multicountry perspective on cultural competence among baccalaureate nursing students. Journal of Nursing Scholarship, 50(1), 92-101. https://doi.org/10.1111/jnu.12350

Cupelli, L. (2016). An innovative service-learning project to develop cultural competency in undergraduate nursing students. Teaching and Learning in Nursing, 11(3), 113-117. https://doi.org/10.1016/j.teln.2016.01.004

D’Andrea, M., Daniels, J., \& Heck, R. (1991). Evaluating the impact of multicultural counseling training. Journal of Counseling and Development, 70(1), 143-150. https://doi.org/10.1002/j.1556-6676.1991.tb01576.x

Deardorff, D. (2015). Intercultural competence: Mapping the future research agenda. International Journal of Intercultural Relations, 48, 3-5. https://doi.org/10.1016/j.ijintrel.2015.03.002

De Beer, J., \& Chipps, J. (2014). A survey of cultural competence of critical care nurses in KwaZulu-Natal. South African Journal of Critical Care, 30(2), 50-54. https://doi.org/10.7196/sajcc.188

Diaz, C., Clarke, P. N., \& Gatua, M. W. (2015). Cultural competence in rural nursing education: Are we there yet? Nursing Education Perspektives, 36(1), 22-26. https://doi.org/10.5480/12-1066.1

Die Bundesversammlung der Schweizerischen Eidgenossenschaft. (2014). Bundesgesetz über den Datenschutz (DSG). Retrieved from https://www.admin.ch/opc/de/classified-compilation/19920153/index.html\#a13 Direct Democracy. (2005). Switzerland's direct democracy. Retrieved from http://direct- 
democracy.geschichte-schweiz.ch/

Diversity. (n.d.). In Merriam Webster's Learning Dictionary. Retrieved from http://www.merriam-webster.com/dictionary/diversity

Doorenbos, A. Z., Schim, M. S., Benkert, R., \& Borse, N. N. (2005). Psychometric evaluation of the cultural competence assessment instrument among health care providers. Nursing Research, 54(5), 324-330. https://doi.org/10.1097/00006199200509000-00006

Dudas, K. I. (2012). Cultural competence: An evolutionary concept analysis. Nursing Education Perspectives, 33(5), 317-321. https://doi.org/10.5480/1536-502633.5 .317

Elliott, C., Adams, R. J., \& Sockalingam, S. (2018). Stages of intercultural sensitivity. Retrieved from: http://www.awesomelibrary.org/multiculturaltoolkit-stages.html Engineering Statistics Handbook. (2018). Measures of skewness and kurtosis. Retrieved from http://www.itl.nist.gov/div898/handbook/eda/section3/eda35b.htm

Fawcett, J., \& Garity, J. (2009). Chapter 6: Evaluation of middle-range theories. In F. A. Davis (Ed.), Evaluating research for evidence-based nursing (pp. 73-88). Philadelphia, PA: F. A. Davis.

Frankfort-Nachmias, C., \& Leon-Guerrero, A. (2015). Social statistics for a diverse society $\left(7^{\text {th }}\right.$ ed.). Thousand Oaks, CA: Sage Publications.

Gallagher, R. W., \& Polanin, J. R. (2015). A meta-analysis of educational interventions designed to enhance cultural competence in professional nurses and nursing students. Nurse Education Today, 35(2), 333-340. 
https://doi.org/10.1016/j.nedt.2014.10.021

Garneau, A. B., \& Pepin, J. (2015). A constructivist theoretical proposition of cultural competence development in nursing. Nurse Education Today, 35(11), 1062-1068. https://doi.org/10.1016/j.nedt.2015.05.019

German Medical Science (2014). Jahrestagung der Gesellschaft für medizinische Ausbildung (GMA) - Poster: Bereitschaft zu interprofessionellem Lernen bei Studierenden der Medizin und der Pflege. Retrieved from http://www.egms.de/static/de/meetings/gma2014/14gma155.shtml

Giger, J. N., \& Davidhizar, R. E. (2002). Giger and Davidhizar transcultural assessment model. Journal of Transcultural Nursing, 13(3), 185-188. https://doi.org/10.1177/10459602013003004

Giger, J., Davidhizar, R. E., Purnell, L., Harden, J. T., Phillips, J., Strickland, O., \& American Academy of Nursing (2007). American academy of nursing expert panel report: Developing cultural competence to eliminate health disparities in ethnic minorities and other vulnerable populations. Journal of Transcultural Nursing, 18(2), 95-102. https://doi.org/10.1177/1043659606298618

Guba, E. G., \& Lincoln, Y. S. (1994). Competing paradigms in qualitative research. In N. K. Denzin \& Y. S. Lincoln (Eds.), Handbook of qualitative research (pp. 105117). Thousand Oaks, CA: Sage.

Halm, M. A., \& Wilgus, E. (2013). Impacting the Latino birthing experience with a cultural competence training program. Online Journal of Cultural Competence in Nursing and Healthcare, 3(2), 1-15. https://doi.org/10.9730/ojccnh.org/v3n2a1 
Halm, M. A., Evans, R., Wittenberg, A., \& Wilgus, E. (2012). Broadening cultural sensitivity at the end of life. Holistic Nursing Practice, 26(6), 335-349. https://doi.org/10.1097/hnp.0b013e31826ed0a7

Hammer, M. R., Bennett, M. J., \& Wiseman, R. (2003). Measuring intercultural sensitivity: The Intercultural Development Inventory. In R. M. Paige (Guest Ed.). Special issue on the intercultural development. International Journal of Intercultural Relations, 27(4), 421-443. https://doi.org/10.1016/s01471767(03)00032-4

Hammer, M. R. (2008). The Intercultural Development Inventory: An approach for assessing and building intercultural competence. In M. A. Moodian (Ed.), Contemporary leadership and intercultural competence: Exploring the crosscultural dynamics within organizations (pp. 203-217). Thousand Oaks, CA: Sage.

Hammer, M. R. (2010). IDI validity. Retrieved from https://idiinventory.com/wpcontent/uploads/2014/01/idi_validity.pdf

Hammer, M. R. (2011). Additional cross-cultural validity testing of the Intercultural Development Inventory. International Journal of Intercultural Relations, 35(4), 474-487. https://doi.org/10.1016/j.ijintrel.2011.02.014

Hammer, M. R. (2012). The Intercultural Development Inventory: A new frontier in assessment and development of intercultural competence. In M. Vande Berg, R.M. Paige, \& K.H. Lou (Eds.), Student learning abroad (Ch. 5, pp. 115-136). Sterling, VA: Stylus Publishing.

Hammer, M. R. (2015a). The developmental paradigm for intercultural competence 
research. Retrieved from https://idiinventory.com/wpcontent/uploads/2015/08/The-Development-Paradigm-for-InterculturalCompetence-Research-08-25-2015.pdf

Hammer, M. R. (2015b). Why should you consider using the Intercultural Development Inventory (IDI)? Retrieved from https://idiinventory.com/wpcontent/uploads/2016/05/why-consider-the-idi.pdf

Hammer, M. R. (2015c). Additional cross-cultural validity testing of the intercultural development inventory. Retrieved from https://idiinventory.com/wpcontent/uploads/2014/02/IDI-Validation-article-2011.pdf

Hamre, P. S. E. (2012). Some want to learn more about culture, and some don't: Cultural sensitivity development of baccalaureate nursing students. Retrieved from https://www.proquest.com/

Harder, N. (2018). Determining the effects of simulation on intercultural competency in undergraduate nursing students. Nurse Educator, 43(1), 4-6. https://doi.org/10.1097/nne.0000000000000403

Harrowing, J. N., Gregory, D. M., O’Sullivan, P. S., Lee, B., \& Doolittle, L. (2012). A critical analysis of undergraduate students' cultural immersion experiences. International Nursing Review, 59(4). 494-501. https://doi.org/10.1111/j.14667657.2012.01012.x

Hawala-Druy, S., \& Hill, M. A. (2012). Interdisciplinary: Cultural competency and culturally congruent education for millennials in health professions. Nurse Education Today, 32(7), 772-778. https://doi.org/10.1016/j.nedt.2012.05.002 
Hirani, S. A. A., Richter, S., \& Salami, B. O. (2018). Realism and relativism in the development of nursing as a discipline. Advances in Nursing Science, 41(2), 137144. https://doi.org/10.1097/ans.0000000000000207

Hofstede, G. (1980). Culture 's consequences: International differences in work-related values. Beverly Hills, CA: Sage.

Horvat, L., Horey, D., Romios, P., \& Kis-Rigo, J. (2014). Cultural competence education for health professionals. Cochrane Database of Systematic Reviews, 5(Art. No.: CD009405), 1-98. https://doi.org/10.1002/14651858.cd009405.pub2

Huckabee, M. J., \& Matkin, G. S. (2012). Examining intercultural sensitivity and competency of physician assistant students. Journal of Allied Health Online, 41(3), e55-61. Retrieved from https://www.proquest.com/

IDI. (2018a). The Intercultural Development Inventory (IDI). Retrieved from https://idiinventory.com/products/the-intercultural-development-inventory-idi/

IDI. (2018b). Intercultural Development Inventory v.3 (IDI) - individual profile report. Retrieved from https://idiinventory.com/wp-content/themes/evolution/pdfs/Jose__Exemplar_-_Profile_-_August_2012.pdf

IDI. (2018c). Intercultural Development Inventory v.3 (IDI) - individual intercultural development plan (IDP). Retrieved from https://idiinventory.com/wpcontent/themes/evolution/pdfs/IDP-Exemplar-Jose.pdf

Institute of Medicine. (2002). Unequal treatment: Confronting racial and ethnic disparities in health care. Retrieved from https://www.nap.edu/resource/10260/disparities_providers.pdf 
Institute of Nursing Science at the University of Basel. (2018). Swiss nursing homes human resources project (SHURP). Retrieved from https://shurp.unibas.ch/ International Council of Nursing. (2013). Position statement of the International Council of Nurses: Cultural and linguistic competence. Retrieved from https://www.icn.ch/sites/default/files/inlinefiles/B03_Cultural_Linguistic_Competence.pdf

Intercultural Development Research Institute. (2018). The Developmental Model of Intercultural Sensitivity. Retrieved from https://www.idrinstitute.org/dmis/

Jeffreys, M. R. (2000). Development and psychometric evaluation of the Transcultural Self-Efficacy Tool: A synthesis of findings. Journal of Transcultural Nursing, 11(2), 127-136. https://doi.org/10.1177/104365960001100207

Jeffreys, M. R., \& Dogan, E. (2010). Factor analysis of the Transcultural Self-Efficacy Tool (TSET). Journal of Nursing Measurement, 18(2), 120-139. https://doi.org/10.1891/1061-3749.18.2.120

Kardong-Edgren, S., Cason, C. L., Walsh-Brennan, A. M., Reifsnider, E., Hummel, F., Mancini, M., \& Griffin, C. (2010). Cultural competency of graduating BSN nursing students. Nursing Education Perspectives, 31(5), 278-285. https://doi.org/10.5172/conu.673.28.1-2.37

Koch, J., Philips, J., \& Davidson, P. M. (2015). Is there a relationship between the diversity characteristics of nursing students and their clinical placement experiences? A literature review. Collegian, 22(3), 307-318. https://doi.org/10.1016/j.colegn.2014.03.007 
Kruse, J. A., Didion, J., \& Perzynski, K. (2014). Utilizing the Intercultural Development Inventory to develop intercultural competence. SpringerPlus, 3(334), 1-8. https://doi.org/10.1186/2193-1801-3-334

Kumas-Tan, Z., Beagan, B., Loppie, C., MacLeod, A., \& Frank, B. (2007). Measures of cultural competence: Examining hidden assumptions. Academic Medicine, 82(6), 548-557. https://doi.org/10.1097/ACM.0b013e3180555a2d

Larson, K. S. (2011). Intercultural sensitivity of nursing faculty. Capella University, Retrieved from https://www.proquest.com/

Leininger, M. M. (1971). Nursing and anthropology: Two worlds to blend. Hoboken, NJ: John Wiley \& Sons Inc.

Leininger, M. M. (1978). Transcultural nursing: Theories, research, and practice (2nd ed.). New York, NY: John Wiley.

Leininger, M. M. (1991). The theory of culture care diversity and universality. In M. M. Leininger (Ed.). Culture care diversity and universality: Theory of nursing (pp. 568). New York, NY: National League for Nursing.

Leininger, M. M. (2002a). Culture care theory: A major contribution to advance transcultural nursing knowledge and practices. Journal of Transcultural Nursing, 13, 189-192. https://doi.org/10.1177/10459602013003005

Leininger, M. M. (2002b). Essential transcultural nursing care concepts, principles, examples, and policy statements. In M. M. Leininger \& M. R. McFarland (Eds.), Transcultural nursing: Concepts, theories, research, \& practice (3rd ed., pp. 4569). New York, NY: McGraw-Hill. 
Leininger, M. M. (2006). Culture care diversity and universality theory and evolution of the ethno nursing method. In M. M. Leininger \& M. R. McFarland (Eds.). Culture care diversity and universality: A worldwide nursing theory (2nd ed., pp. 1-41). Sudbury, MA: Jones and Bartlett.

Leonard, T. (2006). Exploring diversity in nursing education. Journal of Cultural Diversity, 13(2), 87-96. Retrieved from http://europepmc.org/

Lonneman, W. (2015). Teaching strategies to increase cultural awareness in nursing students. Nurse Educator, 40(6), 285-288.

https://doi.org/10.1097/nne.0000000000000175

Mahrer, R. (2011). Patterns of teamwork in nursing teams: an observational study of teamwork between nurses and ASSC-FAGE. Retrieved from http://www.stiftungpflegewissenschaft.ch/fileadmin/user_upload/Projektabstrakt_Mahrer_129793581 3.pdf

Mareno, L., \& Hart, P. L. (2014). Cultural competency among nurses with undergraduate and graduate degrees: Implications for nursing education. Nursing Education Perspectives, 35(2), 83-88. https://doi.org/10.5480/12-834.1

McCambridge, J., Kyprib, K., \& Elbourne, D. (2014). In randomization we trust? There are overlooked problems in experimenting with people in behavioral intervention trials. Journal of Clinical Epidemiology 67(3), 247-253. https://doi.org/10.1016/j.jclinepi.2013.09.004

McClimens, A., Brewster, J., \& Lewis, R. (2014). Recognizing and respecting patients' cultural diversity. Nursing Standard, 28(28), 45-52. 
https://doi.org/10.7748/ns2014.03.28.28.45.e8148

McEwen, M., \& Wills, E. M. (2014). Theoretical basis for nursing (4th ed.). Philadelphia, PA: Wolters Kluwer Health.

McHugh, M. L. (2013). The Chi-square test of independence. Biochemia Medica, 23(2), 143-149. https://doi.org/10.11613/bm.2013.018

Migration Crisis: Swiss are 'too restrictive’ over Syrian refugees. (2015, September 20) Retrieved from http://www.swissinfo.ch/eng

Munoz, C. C., DoBroka, C. C., \& Mohammad, S. (2009). Development of a multidisciplinary course in cultural competence for nursing and human service professions. Journal of Nursing Education, 48(9), 495-503. https://doi.org/10.3928/01484834-20090610-03

National League for Nursing. (n.d.). NLN nurse educator shortage fact sheet. Retrieved from http://www.nln.org/docs/default-source/advocacy-public-policy/nursefaculty-shortage-fact-sheet-pdf.pdf?sfvrsn=0

Nguyen, D-Q., \& Mariani, D. (2014, November 27). Swiss population getting larger, older, more diverse. Retrieved from https://www.swissinfo.ch/engObsan. (2008). Health in Switzerland - national health report 2008. Retrieved from https://www.obsan.admin.ch/sites/default/files/publications/2015/gb-kurzfassunge.pdf

Obsan. (2015). The national health report: The health care system geared toward chronic diseases. Retrieved from https://www.obsan.admin.ch/sites/default/files/uploads/0353-1507-90_0.pdf 
Obsan. (2016). Gesundheitspersonal in der Schweiz - Bestandesaufnahme und Prognosen bis 2030. Retrieved from https://www.obsan.admin.ch/sites/default/files/publications/2017/obsan_71_beric ht_korr.pdf

OdASanté. (2016). Rahmenlehrplan der Bildungsgänge der Höheren Fachschulen Pflege mit dem geschützten Titel dipl. Pflegefachfrau HF / dipl. Pflegefachmann HF. Retrieved from https://www.odasante.ch/fileadmin/odasante.ch/docs/Hoehere_Berufsbildung_und _Hochschulen/RLP_Pflege_HF_09.11._2016-d.pdf

OdASanté. (2018). Bildungssystematik. Retrieved from https://www.odasante.ch/bildungssystematik/

Pahor, M., \& Rasmussen, B. H. (2009). How does culture show? A case study of an international and interprofessional course in palliative care. Journal of Interprofessional Care, 23(5), 474-485.

https://doi.org/10.1080/13561820903163512

Paige, M. R., Jacobs-Cassuto, M., Yershova, Y. A., \& DeJaeghere, J. (2003). Assessing intercultural sensitivity: an empirical analysis of the Hammer and Bennett Intercultural Development Inventory. International Journal of Intercultural Relations, 27(4), 467-486. https://doi.org/10.1016/S0147-1767(03)00034-8

Pallant, J. (2016). SPSS Survival Manual (6 $6^{\text {th }}$ ed.). New York, NY: Mc Graw Hill Education.

Papadopoulos, I., Tilki, M., \& Ayling, S. (2008). Cultural competence in action for 
CAMHS: Development of a cultural competence assessment tool and training programme. Contemporary Nurse, 28, 129-140.

https://doi.org/10.5172/conu.673.28.1-2.129

Papadopoulos, I. (2008). The Papadopoulos, Tilki and Taylor Model for Developing

Cultural Competence. Retrieved from http://ieneproject.eu/download/Outputs/intercultural\%20model.pdf

Peiying, N., Goddard, T., Gribble, N., \& Pickard, Ch. (2012). International placements increase the cultural sensitivity and competency of professional health students: A quantitative and qualitative study. Journal of Physical Therapy Education, 26(1), 61-68. https://doi.org/10.1097/00001416-201210000-00011

Perng, S. J., \& Watson, R. (2012). Construct validation of the nurse cultural competence scale: A hierarchy of abilities. Journal of Clinical Nursing, 21(11-12), 16781684. https://doi.org/10.1111/j.1365-2702.

Purnell, L. (2000). A description of the Purnell model for cultural competence. Journal of Transcultural Nursing, 11(1), 40-46. https://doi.org/10.1177/104365960001100107

Purnell, L. (2002). The Purnell model for cultural competence. Journal of Transcultural Nursing, 13(3), 193-196. https://doi.org/10.1177/10459602013003006

Racism is also a reality in Switzerland. (2016). Retrieved from http://www.humanrights.ch/en/switzerland/internal-affairs/racism/studies/racisma-reality-switzerland-4552

Repo, H., Salminen, L., \& Leino-Kilpi, H. (2017). The cultural competence of graduating 
nursing students. Journal of Transcultural Nursing, 28(1), 98-107.

https://doi.org/10.1177/1043659616632046

Riley, D., Smyer, T., \& York, N. (2012). Cultural competence of practicing nurses entering an RN-BSN program. Nursing Education Perspectives, 33(6), 381-385. https://doi.org/10.5480/1536-5026-33.6.381

Rolls, C., Inglis, A., \& Kristy, S. (2014). The impact on attitudes toward cultural difference of participation in a health focused study abroad program.

Contemporary Nurse, 9(3-4), 246-255. https://doi.org/10.5172/conu.2000.9.34.246

Rottig, D., \& Reus, T. H. (2018). Research on culture and international acquisition performance: A critical evaluation and new directions. International Studies of Management \& Organization, 48(1), 3-42. https://doi.org/10.1080/00208825.2018.1407082

Salvucci, Ch., \& Lawless, C. A. (2016). Nursing faculty diversity: Barriers and perceptions on recruitment, hiring and retention. Journal of Cultural Diversity, 23(2), 65-75. https://doi.org/10.1037/1099-9809.5.4.308

Schouler-Ocak, M., Graef-Calliess, I. T., Tarricone, I. Qureshi, A., Kastrup, M. C., \& Bhugra, D. (2015). EPA guidance on cultural competence training. European Psychiatry, 30(3), 431-440. https://doi.org/10.1016/j.eurpsy.2015.01.012

Schuessler, J. B., Wilder, B., \& Byrd, L. W. (2012). Reflective journaling and development of cultural humility in students. Nursing Education Perspectives, 33(2), 96-99. https://doi.org/10.5480/1536-5026-33.2.96 
Schweizerische Volkspartei SVP. (2010). Volksinitiative "für die Ausschaffung krimineller Ausländer (Ausschaffungsinitiative)”. Retrieved from http://www.ausschaffungsinitiative.ch/de/

Seright, T. (2007). Perspectives of registered nurse cultural competence in rural state e part II. Online Journal of Rural Nursing and Health Care 7(1), 57-69. https://doi.org/10.14574/ojrnhc.v7i1.144

Siegel, S. (1956). Nonparametric statistics for the behavioral sciences. New York, NY: McGraw-Hill.

Simon, M. K., \& Goes, J. (2013). Ex post facto research. Retrieved from http://www.dissertationrecipes.com/wp-content/uploads/2011/04/Ex-Post-Factoresearch.pdf

Sitzman, K. L., \& Eichelberger, W. L. (2011). Chapter 15 Madeleine Leininger's culture care diversity and universality care. In K. L. Sitzman \& W. L. Eichelberger (Ed.). Understanding the work of nurse theorists: A creative beginning (2nd ed., pp. 9398). Retrieved from: http://nursing.jbpub.com/sitzman/ch15pdf.pdf

Spitzberg, B. H., \& Changnon, G. (2009). Conceptualizing intercultural competence. In D. K. Deardorff (Ed.). The SAGE handbook of intercultural competence (pp. $2-$ 52). Los Angeles, CA: SAGE. E.R.

Stein, S., de Andreotti, V. O., Bruce, J., \& Susa, R. (2016). Toward different conversations about the internationalization of higher education. Comparative and International Education, 45(1), 1-18. https://doi.org/10.1007/978-981-287-5327_479-1 
State Secretariat for Education Research and Innovation. (2018). Vocational education and training. Retrieved from https://www.sbfi.admin.ch/sbfi/en/home/services/publications/vocationaleducation-and-training.html

Statista. (2018). Foreign-born population of Switzerland from 2011 to 2016. Retrieved from https://www.statista.com/statistics/548978/foreign-born-population-ofswitzerland/

Sullivan Commission. (2017). Missing persons: Minorities in the health professions. Retrieved from http://www.aacnnursing.org/Portals/42/Diversity/SullivanReport.pdf?ver=201708-02-165732-040

Swiss Confederation. (2017a). Anteil der ständigen ausländischen Wohnbevölkerung (Percentages of the permanent foreign population in Switzerland, 1900-2016). Retrieved from https://www.bfs.admin.ch/bfs/en/home/statistics/cataloguesdatabases/graphs.assetdetail.3302662.html

Swiss Confederation. (2017b). Switzerland's population 2016. Retrieved from https://www.bfs.admin.ch/bfs/en/home/statistics/population/effectifchange/population.assetdetail.3902101.html

Swiss Confederation. (2018a). Federal statistical office - migration and integrationforeign population. Retrieved from https://www.bfs.admin.ch/bfs/en/home/statistics/population/migrationintegration/foreign.html 
Swiss Confederation. (2018b). Main languages of the permanent resident population, 1970-2016. Retrieved from https://www.bfs.admin.ch/bfs/de/home/statistiken/bevoelkerung/sprachenreligionen.assetdetail.4542476.html

Swiss Confederation. (2018c). Religions of the Swiss population in the years 2014-2016. Retrieved from https://www.bfs.admin.ch/bfs/de/home/statistiken/bevoelkerung/sprachenreligionen/religionen.assetdetail.4842974.html

Swiss Confederation. (2018d). Evolution of religious landscape. Retrieved from https://www.bfs.admin.ch/bfs/de/home/statistiken/bevoelkerung/sprachenreligionen/religionen.assetdetail.4402542.html

Swiss Confederation. (2018e). Gesundheitspersonal und Umgang mit Diversität. Retrieved from https://www.bag.admin.ch/bag/de/home/themen/strategienpolitik/nationale-gesundheitsstrategien/gesundheitlichechancengleichheit/gesundheitspersonal-und-umgang-mit-diversitaet.html

Swiss Confederation. (2018f). Data protection - Switzerland. Retrieved from https://www.edoeb.admin.ch/edoeb/en/home/the-fdpic/links/data-protection--switzerland.html

Swiss Confederation. (2018g). Datenschutz und Forschung im Allgemeinen. Retrieved from https://www.edoeb.admin.ch/edoeb/de/home/datenschutz/statistik--registerund-forschung/forschung/datenschutz-und-forschung-im-allgemeinen.html

Swiss Confederation. (2018h). Eidgenössische Volksinitiative 'für die Ausschaffung 
krimineller Ausländer (Ausschaffungsinitiative)’. Retrieved from

https://www.bk.admin.ch/ch/d/pore/vi/vis357.html

Swiss Confederation. (2018i). Health system. Retrieved from

https://www.bfs.admin.ch/bfs/en/home/statistics/health/health-system.html

Swiss Education. (2018). Tertiary level professional education. Retrieved from https://swisseducation.educa.ch/en/higher-professional-education-and-training

Swiss Red Cross. (2018). Transkulturelle Kompetenz - Professioneller Umgang mit

Vielfalt. Retrieved from https://www.redcross.ch/de/soziale-

integration/migration/professioneller-umgang-mit-vielfalt

Taylor, G., Papadopoulos, I., Dudau, V., Maerten, M., Peltegova, A., \& Ziegler, M.

(2011). Intercultural education of nurses and health professionals in Europe

(IENE). International Nursing Review, 58(2), 188-195.

https://doi.org/10.1111/j.1466-7657.2011.00892.x

Terzuolo, E. R. (2018). Intercultural development in study abroad: Influence of student and program characteristics. International Journal of Intercultural Relations, 65, 86-95. https://doi.org/10.1016/j.ijintrel.2018.05.001

The Cantonal Parliament of the Canton of Bern. (2006). Data Protection Act (CDPA). Retrieved from https://www.jgk.be.ch/jgk/de/index/aufsicht/datenschutz/rechtliche_grundlagen.as setref/dam/documents/JGK/DS/de/DS_Data\%20protection\%20Act\%20of\%20Can ton\%20Berne.pdf

The Intercultural Communication Institute. (2016a). Summer Institute for Intercultural 
Communication. Retrieved from https://intercultural.org/program/siic/

Thesaurus.com (2018). Diversity. Retrieved from

http://www.thesaurus.com/browse/diversity?s=t

University of Bern. (2014). Bereitschaft zu Interprofessionellem Lernen bei Studierenden der Medizin und der Pflege. Retrieved from http://boris.unibe.ch/65780/

Vande Berg, M. (2007). Intervening in the learning of US students abroad. Journal of Studies in International Education, 11(3-4), 392-399. https://doi.org/10.1177/1028315307303924

Vande Berg, M. (2009). Intervening in student learning abroad: A research-based inquiry, Intercultural Education, v20(suppl S1-2), S15-S27. https://doi.org/10.1080/14675980903370821

Vande Berg, M., Balkcum, A, Scheid, M., \& Whalen, B. (2004). A report at the half-way mark: The Georgetown Consortium project. Frontiers: The Interdisciplinary Journal of Study Abroad, X Fall, 101-116. Retrieved from https://www.researchgate.net/publication/234690887_The_Georgetown_Universit y_Consortium_Project_A_Report_at_the_Halfway_Mark

Vande Berg, M., Connor-Linton, J., \& Paige, R. M. (2009). The Georgetown Consortium project: Interventions for student learning abroad. Frontiers: The Interdisciplinary Journal of Study Abroad, 18, 1-76. Retrieved from https://files.eric.ed.gov/fulltext/EJ883690.pdf

Wainwright, S. P. (1997). A new paradigm for nursing: The potential of realism. Journal of Advanced Nursing, 26(6), 1262-1271. https://doi.org/10.1046/j.1365- 
2648.1997.00429.x

Wiley, A. (2017). Validation analysis of the Intercultural Development Inventory (IDI). ACS Ventures. Retrieved from https://idiinventory.com/wpcontent/uploads/2017/12/2017-IDI-Validation-ACS-Validation-Analyses-ResultsPhase-3.pdf

Wurz, J. (2014, February 26). Meeting the urgent need for care in nursing homes. Retrieved from http://www.swissinfo.ch/eng 
Appendix A: Approval to Use the IDI

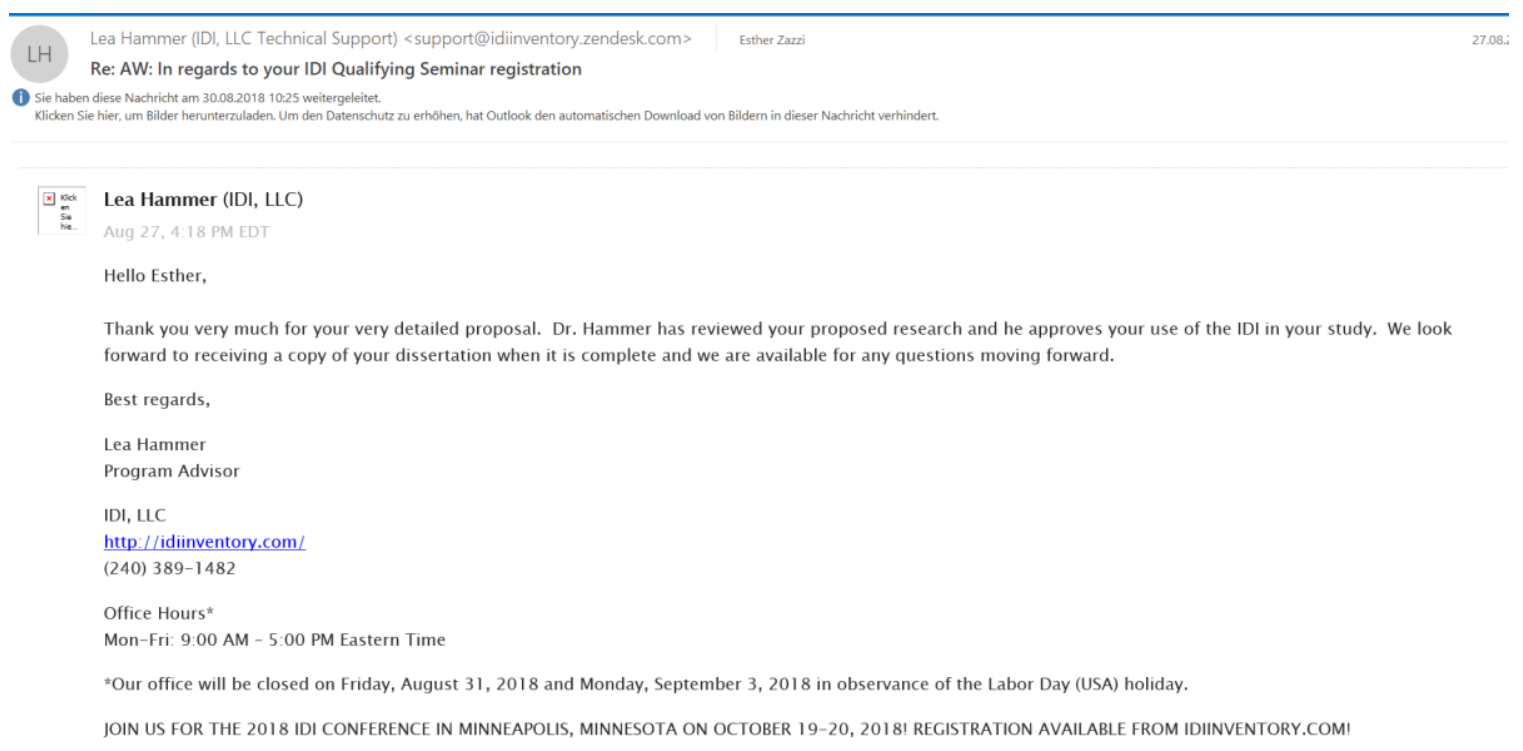


Appendix B: Number of IDI in Each Cohort Including Time-Point

\begin{tabular}{|c|c|c|c|c|c|c|c|c|}
\hline \multirow[b]{2}{*}{ Cohort } & \multirow[b]{2}{*}{ Date } & \multicolumn{2}{|c|}{ start-point } & \multirow[b]{2}{*}{$\begin{array}{l}\text { Response } \\
\text { Rate }\end{array}$} & \multirow[b]{2}{*}{ Date } & \multicolumn{2}{|c|}{ end-point } & \multirow[b]{2}{*}{$\begin{array}{l}\text { Response } \\
\text { Rate }\end{array}$} \\
\hline & & IDI & Students & & & $\mathrm{IDI}^{\odot}$ & Students & \\
\hline 40 & Sep 10 & 40 & 40 & 100 & March 13 & 12 & 36 & 33.3 \\
\hline 41 & Sep 10 & 30 & 36 & 83.3 & March 13 & 24 & 29 & 82.8 \\
\hline 42 & Sep 10 & 43 & 44 & 97.7 & March 13 & 28 & 40 & 70 \\
\hline 43 & Sep 10 & 43 & 43 & 100 & March 13 & 33 & 39 & 84.6 \\
\hline 1 & Sep 10 & 29 & 29 & 100 & Sep 13 & 22 & 22 & 100 \\
\hline 6 & Sep 10 & 43 & 43 & 100 & Sep 13 & 29 & 46 & 63 \\
\hline 7 & Sep 10 & 38 & 38 & 100 & Sep 13 & 25 & 29 & 86.2 \\
\hline 8 & Sep 10 & 49 & 49 & 100 & Sep 13 & 39 & 40 & 97.5 \\
\hline 2 & March 11 & 25 & 26 & 96.2 & March 14 & 20 & 21 & 95.2 \\
\hline 9 & March 11 & 33 & 43 & 76.7 & March 14 & 19 & 39 & 48.7 \\
\hline 44 & Sep 11 & 32 & 43 & 74.4 & March 14 & 35 & 43 & 81.4 \\
\hline 45 & Sep 11 & 42 & 43 & 97.7 & March 14 & 31 & 39 & 79.5 \\
\hline 46 & Sep 11 & 35 & 37 & 94.6 & March 14 & 35 & 37 & 94.6 \\
\hline 3 & Sep 11 & 22 & 28 & 78.6 & Sep 14 & 23 & 23 & 100 \\
\hline 10 & Sep 11 & 50 & 53 & 94.3 & Sep 14 & 44 & 50 & 88 \\
\hline 11 & Sep 11 & 37 & 40 & 92.5 & Sep 14 & 28 & 35 & 80 \\
\hline 4 & March 12 & 22 & 22 & 100 & March 15 & 17 & 18 & 94.4 \\
\hline 12 & March 12 & 33 & 35 & 94.3 & March 15 & 31 & 37 & 83.8 \\
\hline 47 & March 12 & 22 & 22 & 100 & Sep 14 & 24 & 27 & 88.9 \\
\hline 48 & Sep 12 & 39 & 39 & 100 & March 15 & 28 & 34 & 82.4 \\
\hline 49 & Sep 12 & 37 & 37 & 100 & March 15 & 35 & 39 & 89.7 \\
\hline 50 & Sep 12 & 38 & 39 & 97.4 & March 15 & 33 & 37 & 89.2 \\
\hline 13 & Sep 12 & 44 & 56 & 78.6 & Sep 15 & 44 & 50 & 88 \\
\hline 14 & Sep 12 & 40 & 40 & 100 & Sep 15 & 21 & 32 & 65.6 \\
\hline 37 & Sep 12 & 13 & 13 & 100 & Sep 16 & 12 & 13 & 92.3 \\
\hline 15 & March 13 & 17 & 17 & 100 & March 16 & 18 & 18 & 100 \\
\hline 16 & March 13 & 26 & 27 & 96.3 & March 16 & 26 & 27 & 96.3 \\
\hline 51 & March 13 & 21 & 21 & 100 & March 15 & 20 & 21 & 95.2 \\
\hline 52 & March 13 & 22 & 22 & 100 & March 15 & 16 & 18 & 88.9 \\
\hline 17 & Sep 13 & 47 & 47 & 100 & Sep 16 & 35 & 44 & 79.5 \\
\hline 18 & Sep 13 & 48 & 48 & 100 & Sep 16 & 41 & 44 & 93.2 \\
\hline 5 & Sep 13 & 6 & 6 & 100 & & & & \\
\hline 38 & Sep 13 & 17 & 17 & 100 & & & & \\
\hline 53 & Sep 13 & 33 & 37 & 89.2 & Sep 15 & 34 & 37 & 91.9 \\
\hline 54 & Sep 13 & 29 & 29 & 100 & Sep 15 & 27 & 29 & 93.1 \\
\hline 55 & Sep 13 & 29 & 29 & 100 & Sep 15 & 25 & 26 & 96.2 \\
\hline 56 & Sep 13 & 39 & 39 & 100 & Sep 15 & 35 & 38 & 92.1 \\
\hline 57 & March 14 & 24 & 24 & 100 & March 16 & 21 & 24 & 87.5 \\
\hline 58 & March 14 & 25 & 25 & 100 & March 16 & 20 & 26 & 76.9 \\
\hline 19 & March 14 & 15 & 15 & 100 & & & & \\
\hline
\end{tabular}




\begin{tabular}{|c|c|c|c|c|c|c|c|c|}
\hline \multirow[b]{2}{*}{ Cohort } & \multirow[b]{2}{*}{ Date } & \multicolumn{2}{|c|}{ start-point } & \multirow[b]{2}{*}{$\begin{array}{l}\text { Response } \\
\text { Rate }\end{array}$} & \multirow[b]{2}{*}{ Date } & \multicolumn{2}{|c|}{ end-point } & \multirow[b]{2}{*}{$\begin{array}{l}\text { s Response } \\
\text { Rate }\end{array}$} \\
\hline & & IDI & Students & & & $\mathrm{IDI}^{\odot}$ & Students & \\
\hline 20 & March 14 & 24 & 24 & 100 & & & & \\
\hline 59 & Sep 14 & 41 & 41 & 100 & Sep 16 & 31 & 35 & 88.6 \\
\hline 60 & Sep 14 & 43 & 44 & 97.7 & Sep 16 & 36 & 42 & 85.7 \\
\hline 61 & Sep 14 & 43 & 43 & 100 & Sep 16 & 35 & 39 & 89.7 \\
\hline 21 & Sep 14 & 42 & 43 & 97.7 & & & & \\
\hline 22 & Sep 14 & 44 & 44 & 100 & & & & \\
\hline 39 & Sep 14 & 19 & 19 & 100 & & & & \\
\hline 75 & Sep 14 & 29 & 29 & 100 & & & & \\
\hline 23 & March 15 & 35 & 35 & 100 & & & & \\
\hline 24 & March 15 & 37 & 38 & 97.4 & & & & \\
\hline 62 & March 15 & 31 & 31 & 100 & & & & \\
\hline 63 & March 15 & 30 & 30 & 100 & & & & \\
\hline 64 & March 15 & 30 & 30 & 100 & & & & \\
\hline 25 & Sep 15 & 20 & 20 & 100 & & & & \\
\hline 28 & Sep 15 & 15 & 16 & 93.8 & & & & \\
\hline 29 & Sep 15 & 37 & 38 & 97.4 & & & & \\
\hline 34 & Sep 15 & 15 & 15 & 100 & & & & \\
\hline 65 & Sep 15 & 28 & 29 & 96.6 & & & & \\
\hline 66 & Sep 15 & 35 & 35 & 100 & & & & \\
\hline 67 & Sep 15 & 23 & 24 & 95.8 & & & & \\
\hline 68 & Sep 15 & 24 & 24 & 100 & & & & \\
\hline 26 & March 16 & 19 & 19 & 100 & & & & \\
\hline 30 & March 16 & 35 & 36 & 97.2 & & & & \\
\hline 31 & March 16 & 22 & 23 & 95.7 & & & & \\
\hline 35 & March 16 & 13 & 13 & 100 & & & & \\
\hline 69 & March 16 & 26 & 26 & 100 & & & & \\
\hline 70 & March 16 & 23 & 23 & 100 & & & & \\
\hline 71 & March 16 & 23 & 23 & 100 & & & & \\
\hline 72 & March 16 & 29 & 29 & 100 & & & & \\
\hline 27 & Sep 16 & 14 & 14 & 100 & & & & \\
\hline 32 & Sep 16 & 39 & 39 & 100 & & & & \\
\hline 33 & Sep 16 & 34 & 34 & 100 & & & & \\
\hline 36 & Sep 16 & 14 & 14 & 100 & & & & \\
\hline 73 & Sep 16 & 36 & 38 & 94.7 & & & & \\
\hline 74 & Sep 16 & 26 & 26 & 100 & & & & \\
\hline 76 & Sep 16 & 23 & 23 & 100 & & & & \\
\hline Total & & 2298 & 2373 & 96.8 & & 1112 & 1323 & 84.1 \\
\hline
\end{tabular}

